\title{
Connected and Autonomous Vehicles for Intelligent Transportation Systems
}

\author{
by
}

Honeyeh Yazdizadeh, M.A.Sc, Carleton University

\author{
A thesis submitted to the \\ Faculty of Graduate Studies and Research \\ in partial fulfillment of the requirements for the degree of \\ Master of Applied Science in Electrical and Computer Engineering
}

Ottawa-Carleton Institute for Electrical and Computer Engineering (OCIECE)

Department of Systems and Computer Engineering

Carleton University

Ottawa, Ontario, Canada

November 2019

(C) Copyright 2019, Honeyeh Yazdizadeh 
The undersigned recommend to the

Faculty of Graduate Studies and Research

acceptance of the thesis

\section{Connected and Autonomous Vehicles for Intelligent Transportation Systems}

Submitted by Honeyeh Yazdizadeh, M.A.Sc., Carleton University in partial fulfillment of the requirements for the degree of

Master of Applied Science in Elecctrical and Computer Engineering

Thesis Supervisors

Professor F. Richard Yu

2019, Carleton University 


\section{Abstract}

In order to overcome the shortage of transportation facilities, different modern technologies in transportation are being proposed and offered by the governmental bodies, companies and research centers. In particular, there is a phenomenal burst of interest in connected and autonomous vehicles, which use advanced information and communication technologies to connect vehicles with various devices, infrastructure, services, and participants.

Despite the potential benefits of connected and autonomous vehicles, significant challenges remain to be addressed before widespread deployment of intelligent transportation systems (ITS) may begin. An important problem is accurate sign recognition in the presence of noise, which might be the result of weather conditions. Moreover, redundancy in data recording can ensure a more reliable detection that further improves the performance of the car. Besides using cameras, LiDARs are also being used in many autonomous vehicles where data fusion may be used as well.

In this thesis, we study and propose solutions to address detection accuracy as well as measures to improve recording redundancy. This work is conducted in three stages. In the first stage, a real-life testbed for examining different algorithms is designed and implemented. A Toyota RAV4 is equipped with necessary software and hardware like camera, LiDAR, communication devices to elevate the system to a reasonable level 
of autonomous driving. The testbed is under ongoing development. The stage one contribution includes interactive experiences in the implementation of the vehicle.

In the second stage, in order to bring a reasonable level of smartness to the vehicle with improved accuracy for safer drive, a neural network-based algorithm is proposed to better recognize all the road and traffic signs based on the real data from Canadian signs. The results show that with the weather condition in Canada that usually results in dirty signs in the wintertime, the proposed RBF based neural networks can recognize the signs even when the signs are subject to $90 \%$ noise. The promising achieved results are compared with the other methods reported in the literature. While using smart methods like neural network based methods, we can expect higher level of autonomous drive. Among them, we may refer to sign recognition where the sign or the camera is rotated, not sharing the same framework which has been experienced in this thesis as well.

As we cannot rely on a mono-data gathering system, in the final stage, the vehicle is equipped with LiDAR system that improves data recording capability. We show that by using a data-preprocessing algorithm, the signs in the Carleton University campus are detected accurately. All the stages are supported by practical and simulation results. 


\section{Acknowledgements}

First and foremost, I would like to thank my supervisors, Professor F. Richard Yu and Doctor Helen Tang, for their advice and guidance throughout the project. I had the honor of having Prof. Shi, Prof. Boukerche and Prof. Atia as my jury in this thesis. Their comments helped me to improve the quality of my thesis in the very last day this course of study.

I would also like to acknowledge all the support received from my dear family (father, mother, sister, and recently my dear husband). Without them, none of this would have been possible.

A special thanks to Ethan Ross, for all his cooperation.

Last but certainly not least, I would like to acknowledge all of my teachers from my childhood primary school up to now and wish them all the best. 


\section{Contents}

$\begin{array}{lll}1 & \text { Introduction } & 1\end{array}$

1.1 Research Overview . . . . . . . . . . . . . . . . . . . 1

1.2 Some Related Background . . . . . . . . . . . . . . . . . . . 4

1.3 Thesis Contributions . . . . . . . . . . . . . . . . . . . . . 9

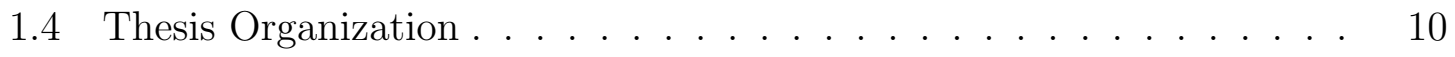

2 Carleton Connected Autonomous Vehicles (CANAL) Hardware and $\begin{array}{ll}\text { Software Design } & 11\end{array}$

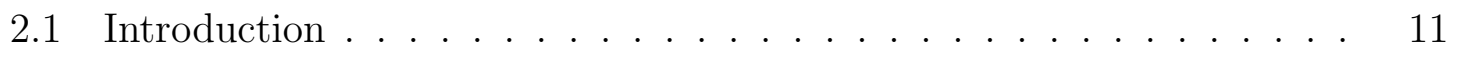

2.2 General Overview . . . . . . . . . . . . . . . . . . . . . . . . 13

2.3 Technical Parts $\ldots \ldots \ldots \ldots \ldots \ldots$

2.3 .1 Comma.AI Openpilot . . . . . . . . . . . . . . . 16

2.3.2 The Dedicated Short Range Communication System (DSRC). 17

2.3.3 Encountered problems with the road and traffic signs in Canada 19

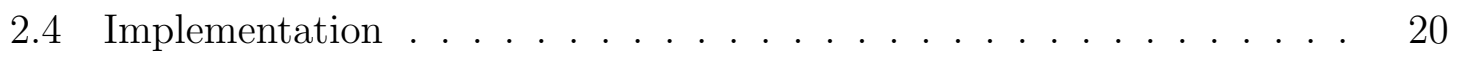

2.4 .1 Toyota RAV4 Implementation $\ldots \ldots \ldots$

2.4 .2 Connected Vehicles Using Cohda Wireless DSRC $\ldots \ldots \ldots 20$

2.4 .3 Autonomous Vehicles Using Comma.AI OpenPilot. . . . . . . 23

2.4 .4 LiDAR in the Testbed $\ldots \ldots \ldots \ldots \ldots$ 
$2.5 \quad$ Experimental Results . . . . . . . . . . . . . . . . . . . . 27

3 Traffic and Road Sign Recognition Using Neural Networks 30

3.1 Introduction . . . . . . . . . . . . . . . . . . . . . . . . . . . . 30

3.2 Pattern Recognition Techniques . . . . . . . . . . . . . . . . . 31

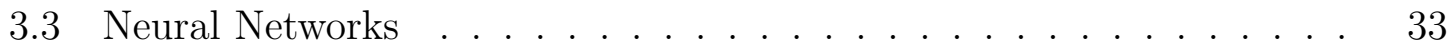

3.4 Learning . . . . . . . . . . . . . . . . . . . . . . . . . . 37

3.5 The proposed Radially Basis Function (RBF) neural network and Generalized Regression . . . . . . . . . . . . . . . . . . . . . . . . . . 38

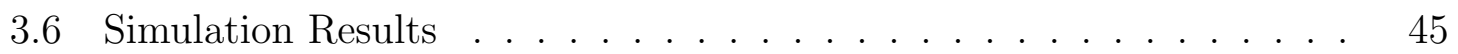

3.6 .1 Basic Simulation Results . . . . . . . . . . . . . . . 45

3.6.2 Comparison . . . . . . . . . . . . . . . 50

3.6 .3 Real Life Situation Scenarios . . . . . . . . . . . . . . . . . . . 59

4 Sign Detection Using LiDAR Recorded Data 69

4.1 Introduction . . . . . . . . . . . . . . . . . . . . . . . . . . . . . . 69

4.2 LiDAR for Mobility Applications . . . . . . . . . . . . . . 70

4.3 LiDAR in Carleton Connected Autonomous Vehicle (CANAL) . . . . 72

4.4 Practical and Simulation Results . . . . . . . . . . . . . . . 75

5 Conclusions and Future works $\quad 80$

5.1 Concluding Remarks . . . . . . . . . . . . . . . . . . 80

5.2 Future Works . . . . . . . . . . . . . . . . . . . . . . . . . . . . . 81

\begin{tabular}{|lr}
\hline A Appendix & 84
\end{tabular}

\begin{tabular}{ll}
\hline References & 112
\end{tabular} 


\section{List of Abbreviations}

ACC Adaptive Cruise Control

AVs Autonomous Vehicles

BPP Backpropagation

CAN Controller Area Network

CANAL Carleton Connected and Autonomous Vehicle

CNN Convolutional Neural Network

CV Connected Vehicle

DIVA Developing Next Generation Intelligent Vehicular Networks And Applications

DSRC Dedicated-Short- Range-Communication

GRNN Generalized Regression Neural Network

ICTCC Information and Communication Technology Council of Canada

ITS Intelligent Transportation Systems

LiDAR Light Detection and Ranging

LKA Lane Keep Assist 
MLP Multi Layer Perceptron

OBD On-Board Diagnostics

OBU On Board Unit

OE Output Error

OED Oxford English Dictionary

RBF Radially Basis Functions

RSU Road Side Unit

SAE Society of Automotive Engineers

SDK Software Development Kit

V2I Vehicle to Infrastructure

V2V Vehicle to Vehicle

V2X Vehicle to Anything

VSIM Vehicles Safety Information Model 


\section{Chapter 1}

\section{Introduction}

\section{$1.1 \quad$ Research Overview}

The world is experiencing a significant population growth. According to recent research, $54 \%$ of people are residing in the cities in comparison with $46 \%$ in rural areas. With the high growth rate in the cities, it is expected the city population increase to $66 \%$ by 2050 [1. Management of a highly populated city is not an easy task because lack of resources imposes many limitations to lifestyle. It seems that conventional methods are not reliable anymore and consequently, employment of modern technologies is necessary to manage a city efficiently. For example, due to the high cost of energy and electricity, the municipality of a megacity has to make sure the lighting system is working efficiently, and energy is preserved for other usages. In such a case, the use of an automatic turning-on/off system for the streetlights is a must.

Among the critical issues in the megacities, we may refer to transportation as a growing problematic issue. Transportation in megacities, in today's life, is a significant problem for all habitats.

In order to overcome the shortage of transportation facilities, different modern 
technologies in the transportation field are being proposed and offered by governmental bodies, companies, and research centers including but not limited to the ongoing research in Carleton University (CANAL) and DIVA in University of Ottawa. In particular, there is a phenomenal burst of interest in connected and autonomous vehicles, that use advanced information and communication technologies to connect vehicles with various devices, infrastructure, services, and participants. Connected and autonomous vehicles have been envisioned to provide enabling key technologies to enhance transportation efficiency, reducing accidents, improving safety, and mitigating the impacts of traffic congestion. The potential of connected and autonomous vehicles has been acknowledged with the establishment of ambitious research programs around the globe [2] [3].

Despite the potential benefits of smart, connected and autonomous vehicles, significant challenges remain to be addressed before widespread deployment of Intelligent Transportation Systems (ITS) 4]. As vehicles become smarter and more connected, they face a range of vulnerabilities. For example, the inherent wireless transmission and high mobility pose special security challenges to smart, connected and autonomous vehicle systems. As a critical infrastructure with inherent vulnerabilities, intelligent transportation systems naturally attract malicious cyber-attacks [5] 6]. There are multiple reports where researchers, hobbyists, and hackers have compromised security keys used by vehicle's ECUs, modified ECU software, and hacked wireless transmissions from vehicle key fobs and tire monitoring sensors [7].

Moreover, such systems in real life are subject to other issues, including different sources of environmental noises. For example, we may refer to noises which are due to weather condition, especially in Canada. In rainy, snow, and fog weather condition, the traffic and road signs cannot be recognized easily.

Therefore, a flexible connected and autonomous vehicle testbed is needed to ad- 
dress the above issues. The testbed needs to be an implementable and user-friendly one so that different algorithms for securing the use of connected and autonomous vehicles can be applied and tested easily.

In this thesis, we introduce a practical solution for implementing a connected and autonomous vehicle testbed that may be used as a building block of an intelligent transportation system in the smart cities in the future. The main plan for testing connected vehicles in Carleton University campus consists of two vehicles, and 12 Dedicated-Short- Range-Communication (DSRC) radios from Cohda Wireless. However, only one of the vehicles contains the hardware necessary for a reasonable level of autonomous driving. This capability is achieved by OpenPilot software created by the Comma.AI that runs on a smartphone with a camera. OpenPilot is able to communicate with the vehicles CAN bus directly to control acceleration over 20mph, braking, and steering. The other vehicle is useful while staging other activities like malicious attacks on the DSRC network. It can also serve as a source of $12 \mathrm{~V}$ power for the Cohda radios and other computer hardware.

The so-called CANAL (Carleton Connected and Autonomous Vehicles) is designed to be used in the Canada environment, so it is important to be smart enough to recognize road and traffic signs in winter weather conditions in Canada. Therefore, a neural network-based methodology is proposed by which the road and traffic sign pictures, with up to $90 \%$ of noise, are recognized. The introduced method is based on one of the efficient neuro-structures called GRNN in which a Radially Basis Function neural network plays the main role. Different scenarios are run in order to show the performance of the proposed method.

Moreover, to be more realistic, the sign detection process is experienced by some data which has been recorded by a LiDAR mounted on top of the car. Having processed the collected data, the signs are also detected in the university campus 
while using LiDAR.

\subsection{Some Related Background}

In this section, some related works about the hardware of the autonomous vehicle are reviewed, although the literature review is completed in each chapter as well. Many Autonomous Vehicles (AVs) have been designed and are under tests in academic and industrial pillars [8]. Towards an integrated, dynamic transportation in a connected society or smart city, connected autonomous (automated) vehicles play the most important role [9].

Looking at the history of autonomous vehicles, we realize that the idea is very old. The first autonomous vehicle was innovated, manufactured, and used during World War II. The invention was called Teetor. It was equipped with a mechanical throttle allowing the driver to set the speed automatically to a desired value. It was invented in 1945 and commercialized in 1958 [8].

Stanford Cart was the second autonomous vehicle that had the chance of landing

on the moon. Although developed and used in 1961, it was similar to today's AV's in the sense that it was equipped with a camera and capable of being programmed for the journey.

Tsukuba Mechanical Engineering System, as the first Japanese car in 1977, was able to recognize street signs, the same as VaMoRs in 1987. This trend has led the ground to today's more advanced cars, like Google's Waymo car and bicycle and Tesla commercial cars with the idea of a full connection with the environment around. Road and traffic sign recognition is among other issues that are studied in this thesis.

In Canada, moreover to the extensive work on CANAL which is fully described in this thesis, we may refer to very valuable research which has been undertaken in 
the University of Ottawa in DIVA [3]. DIVA, stands for Developing Next Generation Intelligent Vehicular Network and Applications, is an excellence center working on different aspects of smart transportation especially on intelligent vehicular networks. The center works under a collaboration of the national and international agencies, industries and universities like NSERC, ITS STI Canada, Ericson, IBM, NOKIA, Cisco, Carleton University, University of British Columbia, Queens University, University of Waterloo, Ecole Polythechnique Montreal and so on. The vision of the center is to develop and deploy distributed, robust, secure and fault-tolerant communication solutions that will enable the proliferation of intelligent vehicular network systems to reduce roadway fatalities, fossil-fuel consumption, greenhouse gas emissions and traffic congestions, while providing drivers and passengers with driving comfort applications such as location-aware services, multimedia streaming, local news, tourist information and alert messages on highways and city streets. The center has done some in deep research in different themes including heterogeneous vehicular networks, intelligent vehicular AdHoc and sensor network, multimedia SOA for VANets, vehicular sensor network security and infrastructure and applications for vehicular communication. Our research work is different from the well done research work in the sense that will be discussed throughout the thesis. Some papers which have been presented in different events by DIVA are referred to in this thesis.

We, at Carleton University, should definitely benefit from all the experiences behind in order to develop Carleton Connected and Autonomous Vehicles (CANAL) step by step.

In Canada, about 2000 fatal accidents happen every year. Information and Communication Technology Council of Canada (ICTCC) expects that the first no human interaction car hits the road by 2022 [8]. This is going to introduce a huge economy to society as it is expected that the market size of the smart cars reach $77 \mathrm{~B} \$$. 
An autonomous vehicle is basically a blend of many modern technologies, mostly in the field of Electrical Engineering. We do not want to ignore the role of mechanical engineering, although combustion motors are being replaced by electrical ones rapidly in an undeniable electrification process in the car manufacturing business. Different disciplines from electrical engineering, including electrical motors, control, and instrumentation as the controlling devices to control speed and position of the cars, communication as the main essence of connectivity are all integrated into a highly connected AV to meet the future of the electrified car industry. They are all, of course, supported by hardware and software computer engineering techniques. Among all, we may refer to the role of artificial intelligence that learns from the acquired data (everything internally from the car and the environment around) in order to increase the level of autonomy.

Light Detection and Ranging (LiDAR) technology has also made a lot of contributions to the field. It may be counted as the main measuring device in the whole loop of control. It helps us to measure the range of the car from other objects on the road, speed and relative speed of the car compared to the other objects and even it may be used to recognize the composition of the object if it is a tree or it is a human being on the road [8]. In this thesis, moreover to the camera data, we work with LiDAR data as well.

Another aspect of computer engineering that contributes to the field is soft computing. The gathered data need a comprehensive data analysis in order to make a decisions for different commands like decreasing speed, stopping the car, or increasing speed and acceleration. Neural network-based methods, including deep learning algorithms and regression methods [10] for both signs recognition and control, are counted as the un-detachable parts of the systems.

Neural networks are being used in different aspects of smart transportation. For 
example, we know that the rapid mobility of vehicles creates a challenging problem in wireless communication. Toward solving this issue, it is needed to anticipate the vehicles movement. The authors in [11] have introduced a recurrent neural network that is capable of performing this task. The proposed neural network accurately predict the handover trigger time using time series quality measurements of the network.

In the communication field itself, many reports are proposing different technologies for data communication. Bluetooth, ZigBee, Radio frequency, Ultra-wideband has been reported in [12]. As the fifth generation of mobile wireless communication, $5 \mathrm{G}$ is essential for every aspect of the smart cities, smart transportation and connected AV's on the ground where a large amount of data is needed to be communicated between vehicles and other things as Vehicle to Anything (V2X).

It is predicted that more than 70 million connected cars will be on the road by 2023 that is a large number of vehicles, although many believe that the maturity is not enough for such a growth [13]. The main obstacle in limiting the growth of such cars includes but not limited to cybersecurity, cost, low level of readiness of road infrastructures, and development of artificial intelligence for a more and practical level of smartness. As mentioned above, cost of transmission in autonomous vehicles is another issue which needs to be addressed as well. An optimized solution has been introduced in [14]. Despite all of these concerns, it is worth noting that many car makers are experiencing development of both traditional and new technology cars. More statistics about the connected and autonomous cars may be found in [13].

In order to come up with the same terminology while studying the matter, a definition of connected cars and automated vehicles is necessary. Connected cars are, basically, cars equipped with sensors, transducers, and communication systems enabling data exchange between the car and other objects like vehicles on the road and roadside infrastructures. It is predicted to have more than 200 sensors in each 
car by 2020. A typical environment of a connected car is shown in Fig 1.1 [12].

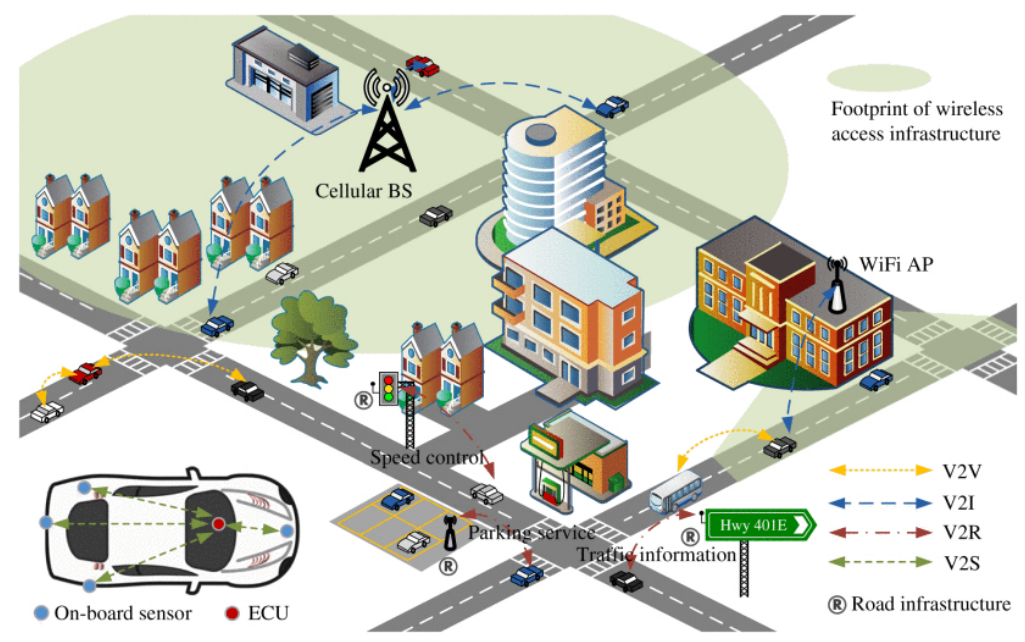

Figure 1.1: Overview of connected vehicles

[12]

In other words, automated vehicles are, basically, those cars that are fully automated and ready for self-driving being capable of operating without direct driver action to control steering, acceleration, and brake [13]. The terms automation is the use of electronics or mechanical devices in order to replace human labor of driving [15]. In some references, autonomous, driverless, or self-driving cars replace the term automated.

Different types of communications between the parties, including Vehicle to Vehicle (V2V), Vehicle to Infrastructure (V2I), I2V, Vehicle to Pedestrian (V2P), and in general Vehicle to Anything (V2X) provides the framework for collision warning and other useful aspects. Depending on the needs of the project, one or more technologies may be used.

Smart industry or industry 4.0 based on different schemes especially Internet of Things is making the future of automation more and more brilliant. Among all, efcient content delivery is one of the fundamental challenges that still need to be 
addressed. The performance of a shared edge caching system for content delivery in smart industry and connected cars applications is studied by the authors in [16]. The proposed mathematical framework considers the distinct content catalogs of the different applications (e.g., industrial and connected cars applications) and content request characteristics from industrial IoT devices and vehicles.

In this thesis, we introduce a combination of the two definitions since CANAL is both automated and receives the necessary data from the environment, including road signs by camera and LiDAR. A synergy between these two concepts paves the way for better smartness in transportation [15].

These types of autonomous vehicles employ different newborn technologies like LiDAR, 5G, and AI (artificial intelligence) in order to explore all the benefits of smart mobility [17] [18, [19].

\subsection{Thesis Contributions}

The main contribution of this thesis is categorized into two parts. The first part comes from a blend of hardware and software design combined with mechatronics. In this part, we designed a hardware and software platform that is mounted on a regular Toyota Rav4. We may call it as general-purpose smart vehicle platform that can be used to implement many algorithms in the field of instrumentation and control, communication, and related issues like data security and attack robustness, machine learning for sign recognition, and so on. In the second part (contribution), we have proposed a specific neural network that is capable of recognizing the road and traffic signs where they are subject to noise and distortion and also where the system is subject to rotation in different conditions. Theses complex have not been

reported all together where other methods like CNN and other methods are employed 
in the references. This is a real-life experience in wintertime in Canada. The second contribution increases the level of autonomy of the CANAL. Toward an even more practical real life atmosphere, sign detection is also performed where the data is collected using a LiDAR.

\subsection{Thesis Organization}

The thesis is organized as follows: An introduction is given in chapter I. Design and implementation of the Carleton Connected Autonomous Vehicle (CANAL), along with the achieved milestones and practical results, are introduced in Chapter II. Chapter III presents the designed neural network solution for road and traffic signs recognition, where they are subject to a very high level of noise, distortion, and rotation in winter weather conditions. Simulation results in different scenarios are also provided. The designed neural network in this chapter works based on the camera pictured data of the sign. To be more realistic, sign detection by LiDAR recorded data is also experienced in this thesis and reported in Chapter IV. The simulation results in this chapter also depict the capabilities of the proposed method. Conclusion, and future works toward a smarter car is given in Chapter $\mathrm{V}$ as the final chapter. 


\section{Chapter 2}

\section{Carleton Connected Autonomous Vehicles (CANAL) Hardware and Software Design}

\section{$2.1 \quad$ Introduction}

Most current activities in intelligent transportation systems, especially in governmentsupported research and development work, have focused on the connected vehicle (CV) and autonomous vehicle (AV) technology and systems. The CV activity relied on the efforts of the past few years to develop and apply dedicated short-range communication (DSRC) that improves road transportation operations.

The essence of smart transportation comes from automation. According to the Oxford English Dictionary (OED), "automation" is "the use of electronic or mechanical devices to replace human labor". In this work, it is applied to some or all of the human interactions for driving. The notion of automation is, sometimes, replaced by "driverless", "self-driving" or "autonomous" in the media, technical reports, and 
papers.

While both "driverless" and "self-driving" imply a complete replacement of the driver in the driving task, most of the systems to which these terms have been applied continue to require a driver to perform some aspects of the driving task. The word "autonomous" which is often used as a synonym for "automated" actually has a completely different meaning that is synonymous with "independence" and "selfsufficiency". Computer scientists have been using this term for several decades to describe systems that have a self-contained decision- making mechanism. However, driving requires a variety of functions beyond decision making, most notably information acquisition function in support of environment perception and recognition function as in human-being. If all functions are performed by self-contained systems in the vehicle, its automation system could be considered to be "autonomous", however, if the vehicles use communications with the infrastructure or other vehicles to acquire information or to negotiate maneuvers, they have "cooperative" automation systems. Cooperative control is among the newly developed methods in control of multivariable and networked control systems. In some cases, "autonomous" has been applied specifically to describe systems that automate all of the driving functions, while in some other cases, it has been applied to all levels of automation. In the California's legislature case, it is used to describe the higher levels of automation technology "to drive a vehicle without the active physical control or continuous monitoring by a human operator" (State of California, 2015). More detailed information in this regard may be found in [15].

With the advent of technology and the availability of intelligent devices, vehicles can be equipped with advanced sensors for autonomous control and communication with other vehicles. It is forecasted that the number of sensors deployed in a vehicle could reach to as many as 200 by 2020 [20]. In CANAL, two important devices for 
receiving data from the environment are camera and LiDAR.

Vehicular transportation is still one of the most important modes of transportation for millions of people in North America and around the world. According to a report, more than 38,000 Americans were killed in 2003, and about 2,700,000 seriously injured in car accidents[21].

Furthermore, each day, millions of person-hours is wasted in traffic jams at red light stops. Therefore, transportation is one of the essential problematic issues in megacities. One aspect of transportation is related to mechanical and electrical engineering as many researchers in the fields are trying to design and manufacture more energy-efficient diesel and electrical engines. In this research work, however, we focus on those ICT-based technologies that are being introduced to help a better quality and more reliable transportation system. Although we are focusing on vehicular transportation, there are many reports related to the other types of transportation as well [22]. Conventional transportation is based on non-autonomous vehicle control, while nowadays, semi-autonomous and autonomous vehicles are becoming more popular in modern cities. Networked vehicles, including Platooning of vehicles, are today known as a promising framework in developing intelligent transportation systems that allow controlling traffic in highways under different conditions. By interconnection of the vehicles and coordination among them, we can potentially enhance safety, increase gas mileage, and reduce emission, in line with the declared goals of smart cities [23].

\subsection{General Overview}

Carleton University, as one of the pioneers in the field of smart cities/smart transportation, is investing a lot in connected autonomous cars. Professor $\mathrm{Yu}$ and his colleagues from academia and industry are working extensively on an autonomous 
vehicle called CANAL. In this thesis, some parts of the mega project, including hardware set up specially working with LiDAR and software set up that includes sign detection and recognition with both camera-based and LiDAR-based data, are studied and proposed. The platform is a Toyota Rav4 that is now being developed systematically toward a smart car. In this chapter, we explain the basics and development of the car as part of this thesis. It is worth noting that car development is work in progress as some other students continue working on the car. Parts of this chapter is extracted from the monthly technical reports that have been documented by the team during the course of this research work [24]

This chapter mainly aims to give readers the required knowledge to understand different aspects of the CANAL. The team has designed the platform for different research and development purposes and applications, such as regular daily usage of the car (move and stop), without using steering and pedals and cybersecurity activities. The future students and researchers who would develop the car toward a standard, safe, secure, functional, and smarter one that responds fairly to the growing demand of industry and other researchers may use this chapter to get familiar with different aspects of the design.

As mentioned before, the CANAL aims to introduce a blend of emerging car technologies with both autonomous and connected cars. By autonomous, we mean the cars that drive autonomously without any human intervention. Many manufacturers, including but not limited to Waymo(formerly Google), Tesla, Audi, BMW, and Mercedes-Benz and Toyota, are working on this technology.

As for standardization, the Society of Automotive Engineers International (SAE) has described the degree of smartness as below:

1. Level 0: there are no autonomous abilities; the system will only provide alerts, but no control. An example of level 0 is distance measuring devices that make 
alerts when the car drives backward, and it approaches an obstacle.

2. Level 1: there is a minimal level of autonomous control, yet, it requires human intervention. As an example, we may refer to Adaptive Cruise Control (ACC), Parking Assist, and Lane Keep Assist (LKA).

3. Level 2: there is a moderate level of autonomy called "hands-off" that allows the system to takes full control of the vehicle's speed and steering, but it may require some human intervention. As an example, Tesla vehicles using the AutoPilot feature belong to this level.

4. Level 3: there is a complete set of hardware and software components that provides full autonomous vehicle control, yet requiring a little human intervention.

5. Level 4: there is a complete set of hardware and software components providing full autonomous with almost no need for human interaction.

6. Level 5: No need for human interaction at all [25].

Different smart algorithms are being introduced and employed in order to reach an acceptable level of autonomy in autonomous vehicles. For example, for vehicle maneuver classification algorithms, Hidden Markov Models are commonly applied for predicting maneuver probability. Authors in [26] have introduces a Convolutional Neural Network (CNN) model for vehicle maneuver classification with relatively high accuracy. A learning strategy that requires only a few samples for training, while maintaining high recognition precision has been introduced in this novel manuscript.

Automation in vehicles has been under development since the late 1900s, with early prototypes demonstrating autonomous abilities up to $30 \mathrm{~km} / \mathrm{h}$. Over the past few years, Tesla has developed an advanced autopilot feature that has full control over all ranges of speed and steering of the vehicle, requiring minimal human intervention 
[27. Audi has offered a new feature named Jam Pilot installed on their 2018 A8 model, which claims to be a level 3 vehicle in slow-moving traffic up to $60 \mathrm{~km} / \mathrm{h}$ on a highway [28] 24]. Waymo currently has successfully developed vehicles on the road, which are entirely self-driving and require no human intervention [29].

\subsection{Technical Parts}

\subsubsection{Comma.AI Openpilot}

Openpilot is an open-source driving system developed by Comma.AI that operates in a semi-operated manner. It operates as a driver-assistance system with the objective of improving visual perception and electromechanical actuator control. It allows users to modify their existing car with increased computing power, enhanced sensors, and continuously-updated driver assistance features that improve with user-submitted data [30]. Android OnePlus 3 has enough capabilities to assist us in employing the Comma.AI by using the software on a cell phone that paves the way for autonomous functions and operation of the vehicle. In addition to the software on the cell phone, a hardware, as shown in Fig 2.1, is required to be mounted on the dash in front of the car. There is no need to use a separate camera. The software uses the cell phone's camera in combination with the vehicles' on-board sensors to control the vehicle. The Comma.AI hardware and software may use algorithms, such as video recognition, artificial intelligence, and machine learning and applies object detection and recognition algorithms to identify external objects, e.g., stop signs, pedestrians, and etc. [31. It is worth mentioning that a specially designed software based on artificial neural networks has been proposed in this thesis as the auxiliary software for road and traffic sign recognition when the signs are subject to bad weather conditions 
during the winter, as it is explained in the next chapter. In this thesis, we have also provided a method for sign detection by using the data gathered from LiDAR as well.

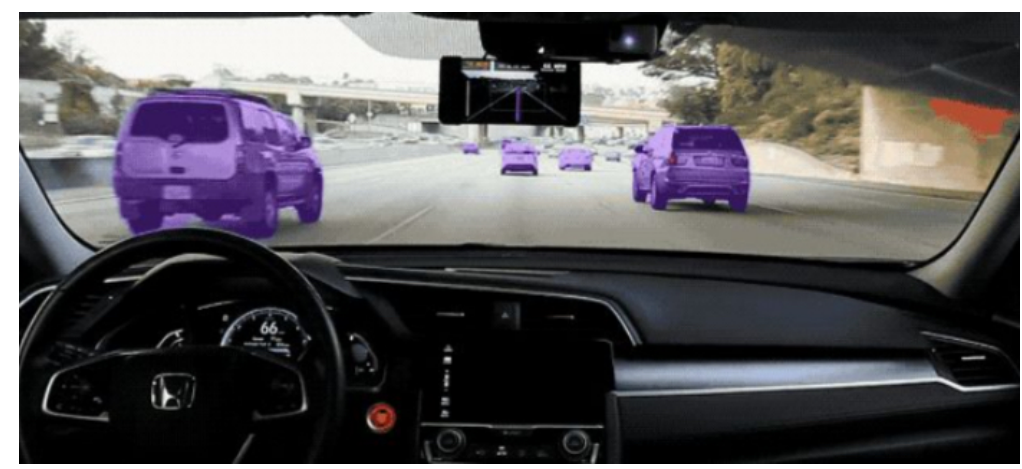

Figure 2.1: The Comma.AI [32]

The Comma.AI is connected to the CAN(Controller Area Network) bus of the car. It provides access to the mechanical parts of the car, including throttle and brake. The Comma.AI helps us to increase the level of autonomy of the car. For instance, by using I-Pedal technology, one can control the gas and the brake functions of the vehicle, allowing controlling the speed and position of the car as needed in real time.

\subsubsection{The Dedicated Short Range Communication System (DSRC)}

As discussed in the introduction chapter, Vehicle to Anything (V2X) communication is one of the essential technology that plays an important role in the field. There have been several methods reported in the literature as the communication platform for smart cars. As such is the Dedicated Short Range Communication System (DSRC) that uses a wireless network infrastructure to enable communication between vehicle-to-vehicle, vehicle-to-road, and in general, vehicle-to-anything else. DSRC is 
composed of two components, namely, the Road Side Units (RSUs) and the On-Board Units (OBUs). Fig 2.2 shows DSRC system and some of its relevant technologies that we used in this big project.

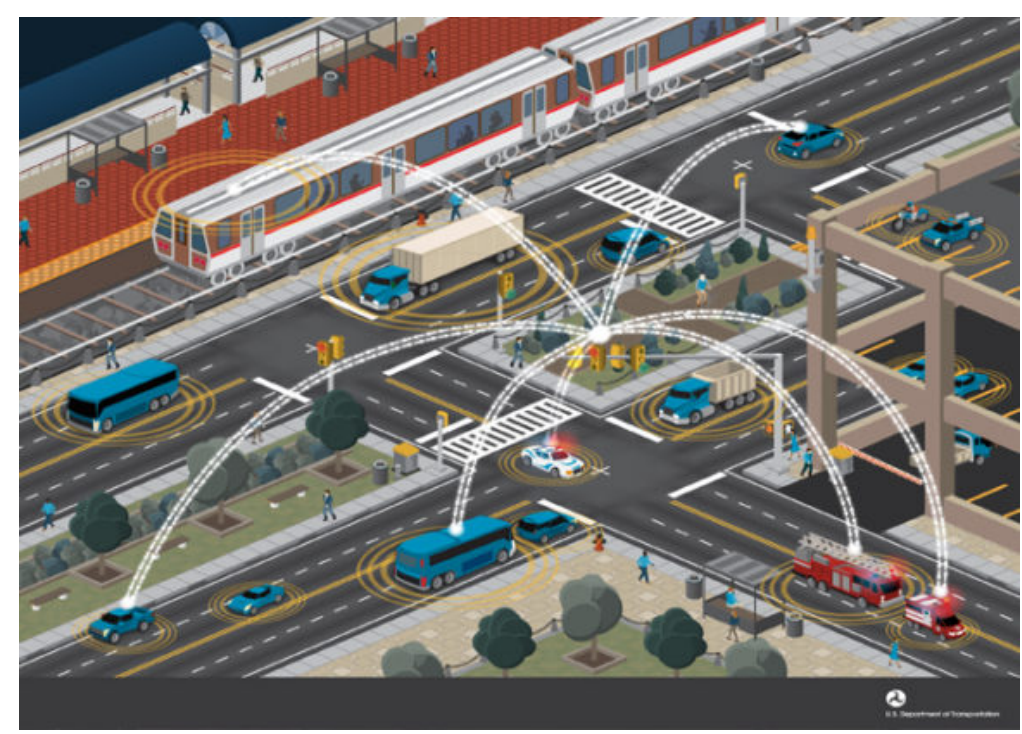

Figure 2.2: The DSRC system.

Similar to the roads and street signs that are placed in visible locations, the RSUs are installed in certain places such as intersections, traffic lights, stop signs, so that they could interact with cars efficiently in sending and receiving data related to traffic across the network. RSUs communicate their locations, locations of nearby emergency cars, location of cars, as well as, safety and security messages, traffic light situation, accidents, emergency stops, and so on.

On the other hand, the OBUs are mounted on each car and provide connectivity to the RSUs and other OBUs via its antenna. As an active part of the network, the OBU may also relay all the information to other nearby vehicles using a predefined protocol that defines authorization policy. The OBU sends and receives information, road status, its location, safety messages, and all other critical data [33].

Three important factors in smart transportation are positioning accuracy, ro- 
bustness and security [34]. In order to improve system security and robustness, the connections among sensor-rich vehicles, common vehicles and roadside units based on a blockchain architecture has been introduced in [34]. In the proposed method if some malicious nodes provide false location information, they will be marked as untrustworthy points and be removed from the blockchain system directly.

In this regard traffic monitoring and location recognition has been an issue in smart transportation systems. As a result different applications have been prepared to be employed to help the drivers for a safer drive. Due to the lack of specialized programming support for such applications DYMES, a dynamic messaging service has been introduced in 35$]$.

\subsubsection{Encountered problems with the road and traffic signs in Canada}

As the CANAL project is intended for Canada, it should adapt its functionality to Canada's weather conditions. Severe weather condition can distort images and potentially cause a false negative and/or false positive results. The most common cause of image distortions is snow covering the objects, rain on the windshield of the vehicle, etc. It is clear that safety is number one issue in using autonomous vehicles since the consequences of failing to recognize a stop sign or a pedestrian could be catastrophic and have detrimental effects on the autonomous vehicle industry [13].

The Comma.AI recognizes a considerable amount of the non-distorted stop signs, while for the distorted ones, only $80 \%$ of the signs can be recognized correctly. This issue referred to "The Stop Sign Problem" or a 80/20 ratio.

This issue possesses a serious security threats to the safety of autonomous vehicles and their drivers. The consequences of not stopping for $20 \%$ of distorted stop signs 
could be detrimental to the drivers and, in turn, to the entire industry. To overcome this issue, we have proposed a neural network-based methodology that is capable of recognizing all the signs even under extreme condition when $90 \%$ added noise and distortion are added as it is discussed in Chapter 3. In addition for a more practical result, we have also shown that the signs may be detected when LiDAR data are recorded and applied for detection.

\subsection{Implementation}

\subsubsection{Toyota RAV4 Implementation}

A regular Toyota RAV4 car is chosen as the basic platform for development of the CANAL. The platform still is under development towards a smarter connected autonomous vehicle. The Comma.AI, the OBU, and its antenna are used as the first components that bring some level of smartness and autonomy to the system. The Comma.AI is mounted on the dashboard. The OBU is connected to the vehicle's on-board computer, and the antenna is mounted on the ceiling of the car. The sensor devices are attached to the Toyota Rav4. Moreover, the car is also equipped with a LiDAR system in order to record the data in a pcab format for further analysis for sign detection.

\subsubsection{Connected Vehicles Using Cohda Wireless DSRC}

One reason for selection of Toyota Rav4 as a testbed is that some of the required features have been provided by the manufacturer. These features are: AC voltage plug, back camera and cruise control.

We enhanced the car with a Cohda Wireless MK5 OBU and Openpilot. The block 


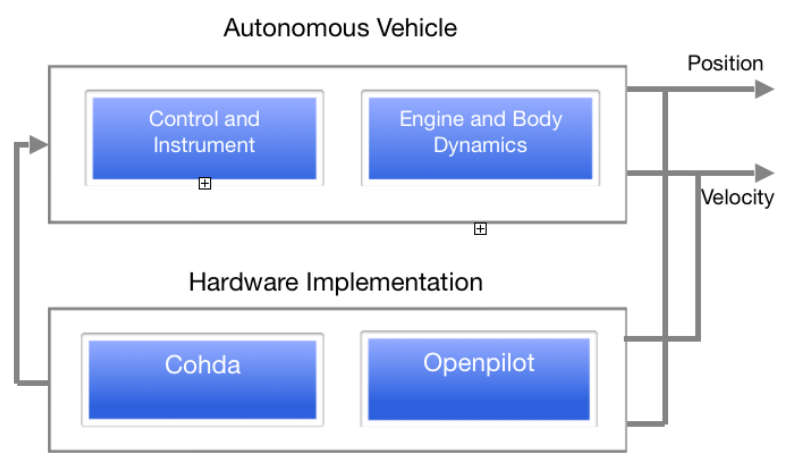

Figure 2.3: The designed block diagram of an autonomous vehicle.

diagram of the vehicle is shown in Fig 2.3 . As shown in the figure, in addition to the body and the engine of the vehicle that governs the dynamic behavior of the car, the vehicle control benefits from enhanced control, Cohda and Openpilot equipments.

By collecting the position and the velocity data from the built-in sensors and measuring devices mounted on the car and feeding them to the hardware unit, the new driving commands is obtained and applied to the car. Continuation of this process in real-time allows the car to be driven without extra aid from the driver. Test results are discussed in the following sections.

Cohda Wireless' 5th generation On-Board Unit (OBU) has received the attention of many researchers working in the field of automotive production as it can be easily deployed for various applications.

Cohda's 5th generation On-Board Unit (OBU) is shown in Fig,2.4. The well known MK5 OBU is basically a small, low-cost, rugged DSRC radio module that can be added to the regular vehicles either for aftermarket deployment or field trials in real life and business applications [36]. It also has all the necessary and sufficient characteristics, including user-friendly and accuracy, to be used as a design reference for automotive production and smart city deployments as well. Fig 2.5 also shown Cohda Wireless' RSU, which is a proper device for outdoor applications. MK5 RSU 


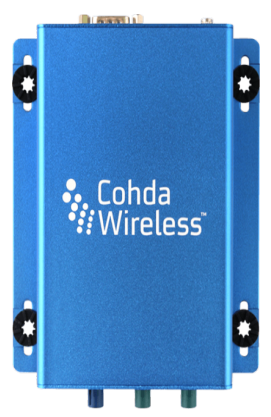

Figure 2.4: Onboard unit (OBU) from Cohda Wireless [36].

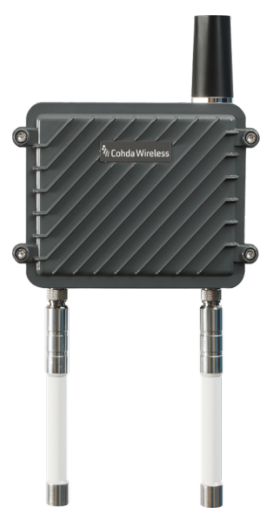

Figure 2.5: Road-Side unit (RSU) from Cohda Wireless [36.

is built with the same chipset as the MK5 OBU. The MK5 Road-Side Unit (RSU) is a rugged outdoor unit with integrated dual antennas housed in a weatherproof enclosure [36].

The MK5 gives the researchers the ability to exchange data at high speeds over extended distances. One of its important characteristics that makes it ideal for autonomous vehicle applications is its unique speed and reaction time. In our case, the test was performed in an outdoor environment where line-of-sight is not an issue. Hence, the testbed benefits from its upgraded performance, and no problem is encountered. By using this hardware, we configured a network of the connected vehicles that were set up for different types of autonomous driving, such as Platoon.

The first step is to set the OBU at the beginning of the drive. Then a configuration 
program called example1609 which has been prepared based on the needs of the project is run to obtain the GPS data of the car. The planned path is recorded by using VSIM, which has been provided by Cohda SDK on a Linux operating system in Oracle's virtualbox. The Cohda SDK is a very helpful and efficient visualization software for the development and testing security issues. By running VSIM, we can monitor vehicles' data in real-time, create our own scenario, and perform the test [37]. This moving car is connected to a stationary car, using another OBU that set in the stationary car, and transmit and receive data through a communication link. So far, we explained how specific hardware might help us to connect the cars via a proper communication channel. In the next section, we explain the other aspect of the project, which is about the needed platform for the software.

\subsubsection{Autonomous Vehicles Using Comma.AI OpenPilot}

Another component that has been used in this project is Openpilot as shown in Fig.2.6. It is an open-source driving agent readily available for use in a research environment. Currently, it performs two functions of Adaptive Cruise Control (ACC) and Lane Keeping Assist System (LKAS) for selected automobile makers as Honda, Toyota, Acura, Lexus, Chevrolet, Hyundai, Kia, Tesla and GM Super Cruise [38.

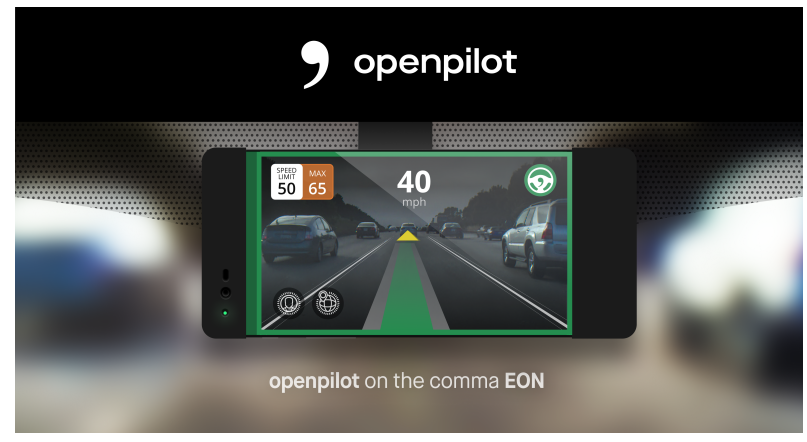

Figure 2.6: Openpilot device [38]. 
Openpilot is used in the proposed hardware shown in Fig.2.6 for autonomous drive. The required steps to setup Openpilot on a Toyota Rav4 car are given as follow:

\section{Required Hardware}

An EON dashcam and a Giraffe are connected to the car via a suitable interface. Giraffe is usually mounted behind the review mirror for a proper plug into the system. It basically provides an easy connection to the car via CANBUS. It has a fake OBD (On-Board Diagnostics) port that allows a user to read and write CAN messages to the CAN bus. Important status signals from accelerator, brake, and etc. are spoofed via CAN bus. The Openpilot device, technically a smartphone with Openpilot software installed, interfaces with the car through the Giraffe with the support of a Panda device. Panda is a universal car interface. It allows you to talk to your car over USB and WiFi [32].

Giraffe has built-in four bit dip-switch that need to be configured so as to pass the factory safety system. The proper setting is 0010. It is worth noting that other setting such as 1011 will not allow sending message to the smart car..

\section{Openpilot Installation}

At the first stage, it is necessary to push the sleep button on the Comma.Ai device and the volume button to see the boot page. Then select the factory reset for a quick reset. Thereafter, we need to reboot it. Having performed the rebooting process, we need to select $\mathrm{Wi}-\mathrm{Fi}$ and enter the URL, which is asked on the screen. The proper address to be entered is: https://openpilot.comma.ai.

3. SSH and Customization to Openpilot

The open-source code for installation can be found on Openpilot's Github. All information related to RAV4 is stored in a .dbc file that is used by Comma.AI 
in order to identify and interpret the cars CAN messages. To enable SSH, our laptop and the Comma.AI device should be on the same Wi-Fi network. We have to enable the SSH on EON dashcam as well. Once SSH enabled, a reboot is required, then we can start working with the EON dashcam and setting it up.

4. Installation in the vehicle

The first step is to install the EON on the front window. Then the Giraffe is plugged in and connected to the panda. To make sure the proper EON is on the dashcam, we run a Python script to check the CAN messages are being read correctly.

5. Calibration and engagement

The calibration begins after we turn on the car and drive above $45 \mathrm{mph}$. Calibration time varies based on the road condition and lightening.

6. Disconnecting driver support ECU To control the brake and acceleration as well, we need to detach the driver support ECU behind the Glove box. Doing so, the car drives between the lanes while adjusting its speed and activate the brake, based on the speed of the leading vehicle. It is worth mentioning that all the driving data are available through a portal called Comma Cabana.

\subsubsection{LiDAR in the Testbed}

The testbed has been augmented to include LiDAR systems from Velodyne and the LiDAR modules from Leddartech to enable connected and autonomous vehicles.

The Velodyne VLP-16 is a capable, well-designed sensor with the state of art technology. The included VeloView by ParaView software produces real-time 3D imagery 
projecting a 360-degree view of terrain surrounding the car. However, the object detection and classification in this software is limited. To improve its performance, new algorithms are developed to determine the required course of action for the car especially when a potential collision scenario is detected.

LeddarTech devices have roughly the same capabilities as the Velodyne sensor. Except, they need to be attached to an electromotor to spin the sensor to achieve 3D capabilities. The casings for the sensors have a silicone conformal coating for protection.

The setup of the VLP-16 on the Rav4 is very straightforward. First, the device is mounted on top of the car and powered by the car's power outlet. An interface cable interconnects the device and provides data communication.

The Velodyne VLP-16 comes in a package containing the LiDAR device, an interface box for the Internet connectivity, power supply and GPS, AC/DC power supply for a 110 AC plug, a one-meter long Ethernet cable and a USB memory stick with Velodyne open-source visualization and recording software Veloview.

One end of the power supply is connected to the interface box and the other end is connected to a power outlet. The sensor is now receiving the power indicating with a green LED in the interface box. One end of the Ethernet cable is plugged to the interface box and the other end connected to the laptop. Then we install the Veloview that is provided in the USB stick. To set up the system, we used manual network setting. Any network address of 192.168.1.X can be used. The exception for X is 0 and 201 and 255. The subnet mask is 255.255.255.0.

To Access the VLP-16 user interface, first, we open a browser and enter 192.168.1.201. Note that we can change this IP address easily on the main menu. The VLP-16 contains a web server that allows the user to monitor configuration parameters, choose network settings, control the rotational speed, and upload new 
firmware and calibration files. The configuration we used in this webserver was the rotational speed that we set to 600 time to make the device work.

Now we can open the Veloview to see a live sensor streaming. We have to click on the File tab, open sensor stream. The sensor configuration window will open, prompting us to choose the correct sensor. We choose VLP-16 and then click OK. We will view a live stream of our sensor. We can click and drag the mouse for different views. It is also possible to click on the record button to start recording the data as a .pcap file. In the following chapters, the recorded data are shown. The .pcap files which are introduced as the dataset show the stop signs located on the Carleton University campus. It is worth mentioning that in a very more advanced level of autonomy and in industrial version of the autonomous vehicle, it is advisable to diffuse both camera and LiDAR data for more accuracy and reliability.

\subsection{Experimental Results}

In this section, the experimental results of the hardware and software implementation in the real-life test on the Toyota Rav4 are presented.

The car has been tested physically in Ottawa. The hardware configuration depicts a very accurate autonomous function. The following figures show how good the car has been derived without any accident or risky behavior. The car was tested on the Carleton Campus after each phase. Fig, 2.7 shows the successful test of connectivity.

Figs 2.8 and 2.9 show the performance of the car where it is tested autonomously. Fig 2.7 is a screenshot from the live video recorded while we were testing the car. The video is available upon request. 


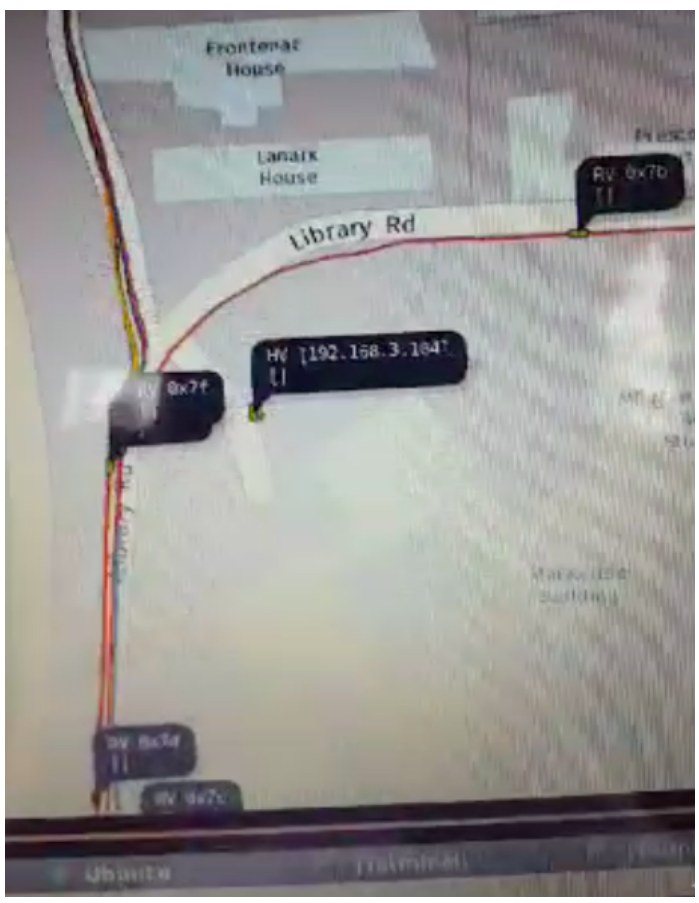

Figure 2.7: Record from VSIM of a live autonomous drive.

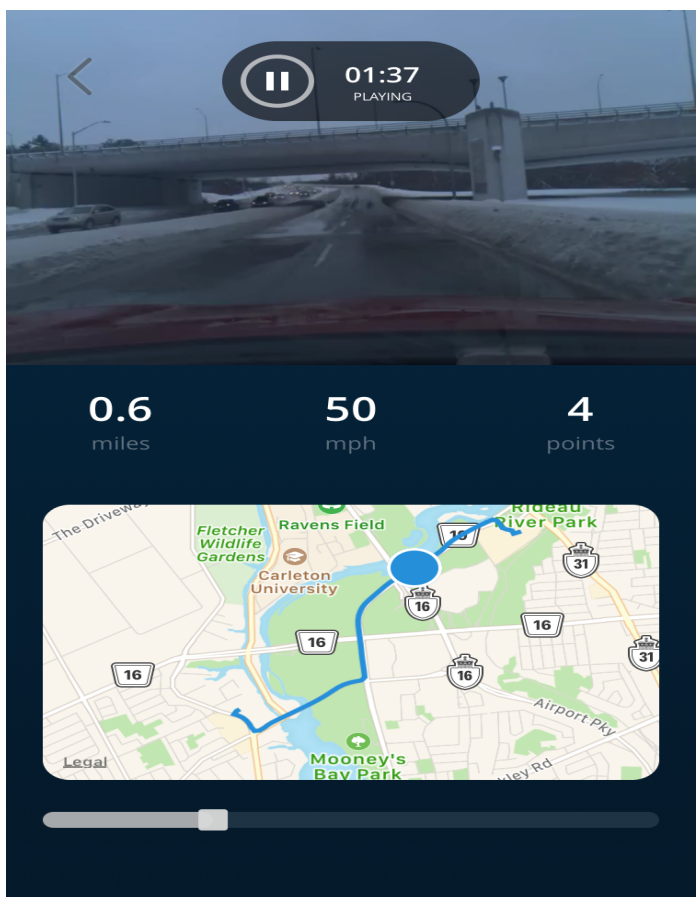

Figure 2.8: Record of driving in Chffr application by OpenPilot. 


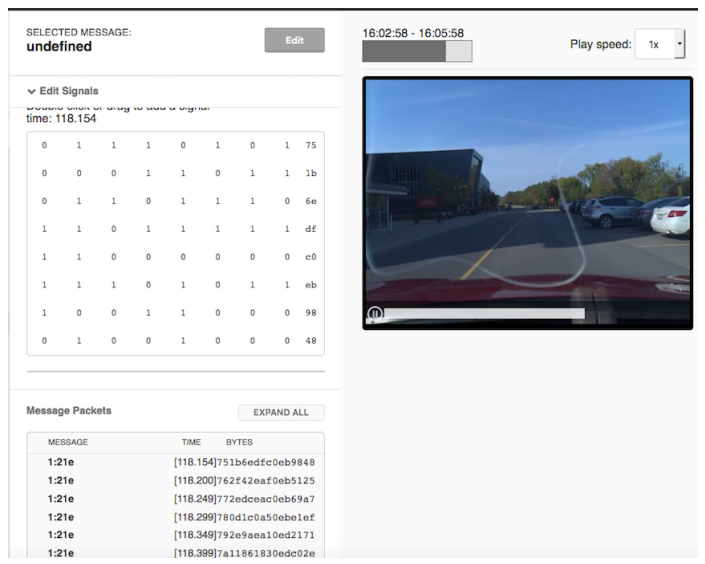

Figure 2.9: Record of autonomous driving in Comma Cabana Portal. 


\section{Chapter 3}

\section{Traffic and Road Sign Recognition Using Neural Networks}

\subsection{Introduction}

From the safety point of view, it is important that an autonomous car has the capability to recognize road signs even under extreme weather conditions. Among many pattern recognition methods, artificial neural networks offer a system with learning ability that can adapt to various conditions. In this chapter, we introduce neural network-based methods for road and traffic sign recognition by using the picture data of different signs.

In broad terms, the objective of pattern recognition known to develop a mathematical or heuristic model that could output a given pattern, e.g., traffic sign, despite impaired input, e.g., noisy or dirty picture of traffic sign. Given the possibility

of noisy input, the solution to the exactly matching a dirty sign to a clean one is extremely hard. Therefore, we aim to obtain a model whose output is as close as possible to the output of the clean one. Different methods have been introduced for 
pattern recognition in the literature. The broad application of pattern recognition includes recognising medical results (ECG, ill-posed tumors, ..), signature, face and fingerprint, and so on [39] [40] [41].

Among many modeling techniques, parameterized modeling is promising as it allows adjustment to model characteristics. The parameters are usually adjusted adaptively so that the recognition task is performed with fewer mistakes [42]. Among parameterized models, neural networks have shown promising results for identification and pattern recognition.

In Canada, during the winter, the traffic and road signs could be dirty or covered with snow. Smart vehicles receive data pictures from the surrounding environment, especially the signs. They analyze the pictures to take the related actions. For example, when it receives a picture of a stop sign, it instruct the vehicle to stop. As a result, it is required to recognize the picture even if it is dirty or snowy. We propose a neural network for recognizing the road and traffic signs in the presence of noise. The performance of the proposed neural network is tested under severe condition with 95\% noise loaded on the picture. Moreover, in real-life applications, the x-axis of the camera mounted on the vehicle may not be aligned with the x-axis of the road signs, that is typically installed horizontally. Therefore, it is required to employ pattern recognition models to recognize the noisy signs even when they are rotated. This greatly enhances the autonomous vehicle's ability to recognize the sign in different conditions. These capabilities may be considered as other contribution of this thesis.

\subsection{Pattern Recognition Techniques}

The proposed methods for pattern recognition are generally classified into two main categories, namely, conventional methods and neural network-based methods. We 
denote pattern recognition methods that do not employ neural networks as conventional ones. The conventional and neural network-based methods are briefly reviewed as follows.

Conventional methods are well established with a simple structure. They can recognize only simple patterns provided that these patterns are not subject to environmental or other types of noises. Conventional methods are very similar to the system identification issue, where the justification for using a more complicated structure really depends on the level of complexity of the system to be identified. A general model for identification of a linear system is given in the well-known methods like ARX, ARMAX, and Output Error(OE) [43].

In conventional methods, a-priori knowledge about the system is usually required in order to recognize the pattern correctly. The random nature of noise and its various types make pattern recognition far more complicated as a-priori knowledge may not be available.

On the other hand, neural network-based methods have shown good results in pattern recognition due to the learning ability to approximate nonlinear maps. We will discuss this ability following a short introduction to neural networks.

Neural networks have shown to be capable of mapping very general class of applications with changing parameters, stochastic behavior and nonlinear characteristics. This property has made the neural networks a very useful candidate for pattern recognition tasks. A neural network basically learns recognition of traffic and road signs under different conditions. Inspired by the approximation theorem [44, neural networks have been applied to many identification problems, including pattern recognition. The success of the neural network in pattern recognition is attributed to three important features:

1. their nonlinear characteristics make them suitable to deal with nonlinearity, 
2. their parallel and pipeline processing characteristics allow them to perform parallel tasks more efficiently and quickly, and

3. their learning characteristics make them ideal for adapting to different environmental conditions.

The wide range of neural network applications (e.g. [45]) can be classified into two categories. The first includes Multi-Layer Perceptron (MLP), Backpropagation (BPP), Radially Basis Functions (RBF), Convolutional Neural Networks (CNN). The second type is dynamic, and it is called re-current neural network in which the equation governing input-output mapping is a differential equation, e.g., Hopfield neural network [46].

\subsection{Neural Networks}

An artificial neuron model based on the biological model of a neuron was proposed by McCulloch and Pitts [44. Fig 3.1 shows the structure of such neuron. The output

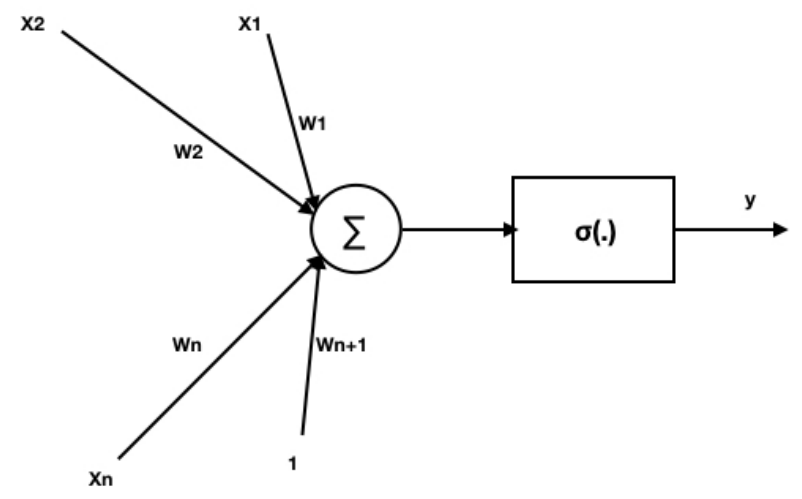

Figure 3.1: Single neuron structure. 
of the neuron is a nonlinear function of the weighted sum of its inputs:

$$
n e t=\sum_{i=1}^{N+1} w(i) x(i), y=\sigma(n e t)
$$

where $x_{i}{ }^{\prime}$ 's are the inputs, $X_{N}$ is the constant input that is called bias, $w_{i}$ 's are the adjustable weights, and $\sigma($.$) is a nonlinear function called activation function. McCul-$ lach and Pitts considered a threshold function as activation function in their proposed neuron model. However, other functions such as sigmoidal functions may also be used [45]. This nonlinearity is the basis for providing the network with the capability to deal with capturing nonlinear phenomena involved in pattern recognition. A network with an arrangement of these neurons can perform different logical and classification tasks as demonstrated in the literature(e.g. 45]).

A single layer neural network is constructed by adding several neurons in parallel. By interconnecting these layers, one may be able to construct a multi-layer feedforward neural network. Fig 3.2 shows a multi-layer feedforward with input, output and hidden layer. Governing equations of a multi-layer feedforward neural network are as follows

$$
\left[\operatorname{net}_{j}^{l}(t)\right]=\sum_{i=1}^{N^{(l-1)}} w_{j i}^{l} o_{i}^{l-1}(t), o_{j}^{l}(t)=\sigma\left(\operatorname{netj}^{l}(t)\right),
$$

where $\sigma($.$) is activation function and it could be any lower-upper bounded mono-$ tonically increasing function, such as sigmoidal function, net $_{j}^{l}(t)$ is the weighted input of the $j^{t h}$ neuron in the $l^{t h}$ layer. $o_{i}^{l-1}(t)$ is the output of $i^{t h}$ neuron in the $(l-1)^{t h}$ layer, $o_{j}^{l}(t)$ is the output of the $j^{t h}$ neuron in the $l^{t h}$ layer, and the $N^{l-1}$ is number of neurons in the $(l-1)^{t h}$ layer. Multi-layer feedforward neural networks should have at least two layers of neurons because it has been shown that a two-layer feedforward neural network is capable of approximating any nonlinear function to an arbitrary 


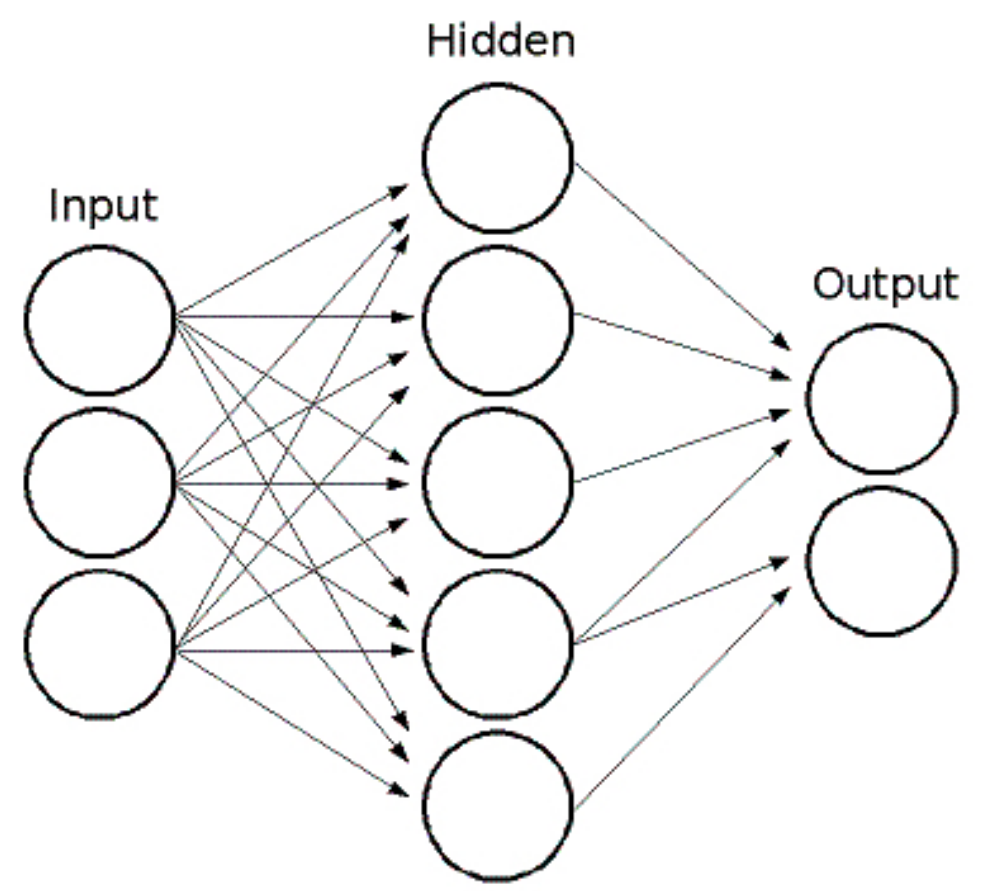

Figure 3.2: Multi-layer neural network [47]

degree of accuracy [44] [45]. Note that Fig 3.2 is considered to be two-layer as the neurons are arranged in hidden and output layer. 
As mentioned before, another type of neural network that is widely used is the recurrent neural network. The first of such dynamic network was introduced by Hopefield [46] by adding a feedback from the outputs of a layer to its inputs or to the inputs of the previous layers.

Unlike static feedforward neural networks whose outputs are functions of their current inputs and the weights, the outputs of the Hopefield network depend on the current and the previous values of the outputs, inputs, and the weights. Fig. 3.3 depicts the structure of a Hopefield neural network.

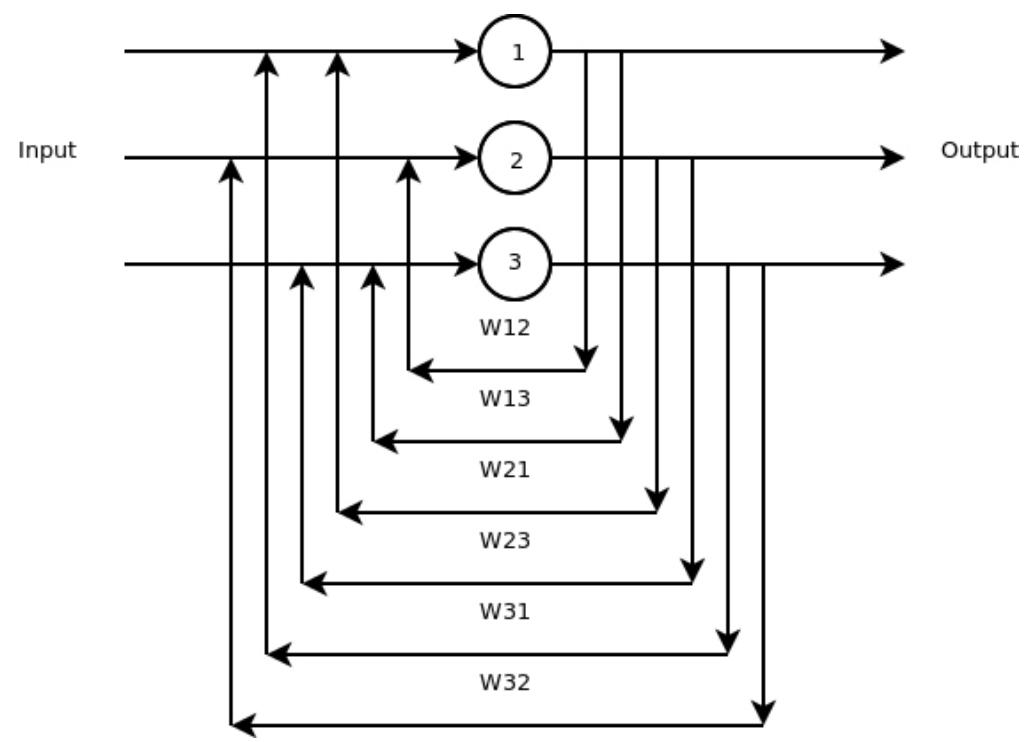

Figure 3.3: Hopefield neural network [48]

Governing equations of such a network is a differential equation rather than an algebraic equation. In all of the above structures, the neural networks deploy an algorithm to learn from a given input-output data set by adjusting the weights during the training period so that the output response closely matches to the training data output. 


\subsection{Learning}

In artificial neural networks, learning or training is based on a procedure through which the weights of the network are adjusted in such a way that the input-output equation of the network produces the desired mapping, i.e., the desired traffic sign. There are two types of learning, supervised and unsupervised. Supervised learning uses an off-line training set consist of input-output values in order to adjust the network parameters. In unsupervised learning, however, there is no desired values for the training set. The learning that is used throughout this thesis is a supervised one.

The aim of the training is to adjust the parameters of the network in order to minimize the output recognition error. This minimization problem is formulated as a standard optimization problem [43]. The nonlinear optimization problem cannot be easily solved analytically. Therefore, a search algorithm is used instead of finding the optimized values of the weights. Steepest descent gradient method is the most popular one, although other algorithms have also reported in the literature.

In the steepest descent algorithm, also called as delta rule, the weights are adjusted in the negative direction of the gradient in the multi-dimensional error surface. The first line of the derivation of the learning rule is:

$$
W^{\text {new }}=W^{\text {old }}-\eta \nabla E(W),
$$

where $\eta$ is a positive constant, known as the learning rate. It has been shown that selection of sufficiently small learning rate can solve many pattern recognition problems. It is because the smaller the learning rate is, the lower is the convergence rate.

The gradient terms, i.e., $\nabla E_{w j i}$ can be computed by using Backpropagation, also, known as the Generalized Delta Rule for off-line training. Backpropagation, in its present form, was introduced by Rumelhart Hinton and Williams even though Parker 
3.5 The proposed Radially Basis Function (RBF) neural network and Generalized

Regression

and Werbos had described very similar procedures [44. In any case, we need a training set to train the network and testing data set in order to evaluate the performance of the trained neural network. In this thesis, the training set is formed by using the original picture along with the noisy version of the pictures. This helps us to prepare a rich training set. To evaluate the performance of the network, again some noisy data are generated by the same method which has been used to prepare the training set. In other words, there is no difference between the procedure of generating training set and evaluation set and this may be considered as one of the advantages of the proposed method that makes it more reliable.

\subsection{The proposed Radially Basis Function (RBF) neural network and Generalized Regression}

Unlike radially basis function neural networks, Multi-layer Perceptron(MLP)with Backpropagation, that use sigmoidal functions, uses radial basis functions as activation functions. The network has basically two layers. In the first layer, the activation functions are radially basis functions, whereas the second layer uses a linear function as the activation function. In other words, a linear combination of radial basis functions of the inputs produces the output. RBF's are used in function approximation, time series prediction, classification, system control, and pattern recognition. Broomhead and Lowe have formulated the RBF for the first time in 1988 [49]. The structure of a RBF neural network is shown in Fig. 3.4. It is a two-layers network where the first layer uses nonlinear RBF activation functions and the second layer uses a linear function. The inputs, $X$ as a vector of real numbers representing the signs picture data $X \in R^{n}$. The output of the network is produced by a scalar function of the 


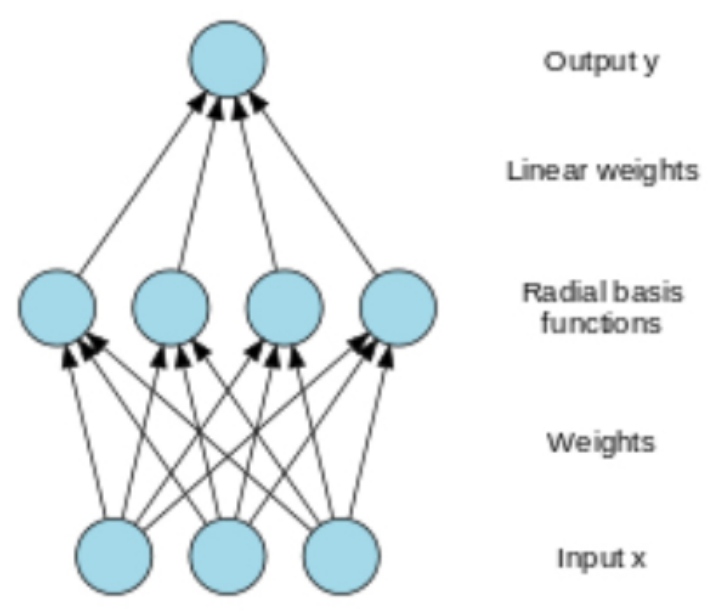

Figure 3.4: Architecture of a radial basis function network [49]

weighted input vector $\varphi: R^{n} \rightarrow R$. In general it can be written as the following:

$$
\varphi(X)=\sum_{i=1}^{N} \alpha_{i} \rho\left(\left\|x-c_{i}\right\|\right)
$$

where $N$ is the number of neurons in hidden layer, $c_{i}$ is the center vector for neuron $i$, and $\alpha_{i}$ is the weight of neuron $i$ in the linear output neuron.

The function $\rho($.$) is radial basis function such as Gaussian as:$

$$
\rho\left(\left\|x-c_{i}\right\|\right)=\exp \left[-\beta\left\|x-c_{i}\right\|^{2}\right]
$$

Similar to MLP and BPP neural networks, RBF neural networks are universal approximators on a compact subset of $R^{n}$, meaning that they are capable of approximating any continuous function on a closed, bounded set with arbitrary precision. The parameters $\alpha_{i}, c_{i}$ and $\beta_{i}$ are adjusted to optimize mapping between $\varphi(X)$ and the data.

Similar to other neural networks, RBF neural networks are trained by using the input-output data set and target values i.e., $x(t), y(t)$ fort $=1, \ldots, T$ by a two-step 
3.5 The proposed Radially Basis Function (RBF) neural network and Generalized

Regression

algorithm.

In the first step, the center vectors $c_{i}$ of the RBF functions in the hidden layer are adjusted. In the second step, a linear model is fit with coefficients $w_{i}$ to the hidden layer's outputs with respect to some objective function. A common objective function, at least for regression/function estimation, is the least squares function:

$$
K(w)=\sum_{t=1}^{T} K_{t}(w),
$$

where

$$
K(w)=\left[y(t)-\varphi(x(t), w]^{2} .\right.
$$

A third optional backpropagation step can be performed to fine-tune all of the RBF parameters [50]. The reason that RBF neural networks are very good candidates for pattern recognition is that they can be used to interpolate a function $y: R^{n} \rightarrow R$ when the values of that function are known on finite number of points: $y\left(x_{i}\right)=b_{i}, i=$ $1, \ldots, N$. Taking the known points $x_{i}$ to be the centers of the radial basis functions and evaluating the values of the basis functions at the other points $g_{i j}=\rho\left(\left\|x_{j}-x_{i}\right\|\right)$ the weights can be solved from the equation

$$
\left[\begin{array}{cccc}
g_{11} & g_{12} & \cdots & g 1 N \\
g_{21} & g_{22} & \cdots & g_{2 N} \\
\vdots & & \ddots & \vdots \\
g_{N 1} & g_{N 2} & \cdots & g_{N N}
\end{array}\right]\left[\begin{array}{c}
w_{1} \\
w_{2} \\
\vdots \\
w_{N}
\end{array}\right]=\left[\begin{array}{c}
b_{1} \\
b_{2} \\
\vdots \\
b_{N}
\end{array}\right]
$$

It can be shown that the interpolation matrix in the above equation is non-singular, if the points $x_{i}$ are distinct, and thus the weights $w$ can be solved by simple linear algebra: 
3.5 The proposed Radially Basis Function (RBF) neural network and Generalized

Regression

$w=G^{-1} b$

where

$G=\left(g_{i j}\right)$.

Steepest descent gradient method may also be used to train the network. In this method, the weights are adjusted in a direction opposite to the gradient of the objective function as follows:

$$
w(t+1)=w(t)-\nu \frac{d}{d w} H_{t}(w)
$$

where $\nu$ is "learning parameter."

For the above introduced network, $a_{i}$, the algorithm becomes [49:

$$
a_{i}(t+1)=a_{i}(t)+\nu[y(t)-\varphi(x(t), w)] \rho\left(\left\|x(t)-c_{i}\right\|\right)
$$

Generalized Regression Neural Network (GRNN) was first proposed by D.F. Specht in 1991 [51]. In his famous paper, he introduced GRNN as a memory-based single-pass neural network that produces estimates of a continuous variable with convergence property. Similar to other neural network structures, it is a highly parallel structure that is capable of dealing with linear and nonlinear mapping. The basic idea comes from the fact that regression of a dependent variable $Y$ on an independent variable $X$ is computed based on the most probable value of $Y$ for each value of $X$ based on a finite number of possibly noisy measurement of $X$ and the associated value of $Y$ to it. In other words, the learning structure finds the best-matched value for a noisy input after training. It is basically why we need to recognize the noisy (dirty) traffic and road signs during the winter time. A two-layer feedforward neural network with a RBF layer connected to a summation layer as the output layer. As for radially basis function, a normalized Gaussian kernel is used in the hidden layer as activation 
3.5 The proposed Radially Basis Function (RBF) neural network and Generalized

functions. After training, GRNN memorizes every unique pattern presented to the network as the training set, which happens to be traffic and road signs pictures as well as their noisy ones in this work.

Many applications have been reported as the use cases of GRNN, including prediction and classification, modeling of different phenomena like power load forecasting, flow forecasting, and many other statistical modelings where the presence of noise is a basic assumption [52] [53].

Each training sample from the training set (the set of pictures of the Canadian road and traffic signs) is given to the network, and the adjustable parameters are adjusted accordingly. GRNN is a single pass neural network, and no error propagation is required. Use of Gaussian functions allow accommodation of noisy inputs at the expense of a larger set of neurons. For instance, in our case for 58 pictures with resolution $86 * 83$, the number of neurons in the first layer is 6138 neurons which is significant when compared to BPNN.

The mathematical representation of the GRNN is given as [54]:

$$
Y(x)=\frac{\sum_{k=1}^{N} y_{k} K\left(x, x_{k}\right)}{\sum_{k=1}^{N} K\left(x, x_{k}\right)}
$$

where $Y(x)$ is the predicted value for the input, $y_{k}$ is the activation weight for the pattern layer neuron at $k, K\left(x, x_{k}\right)$ is the Radial basis function kernel or simply Gaussian kernel given by:

$$
K\left(x, x_{k}\right)=e^{-d_{k} / 2 \sigma^{2}}, \quad d_{k}=\left(x-x_{k}\right)^{T}\left(x-x_{k}\right),
$$

where $d_{k}$ is the squared euclidean distance between the training samples $x_{k}$ and the input $x$. As already mentioned, GRNN has a radial basis layer and a special linear layer [55]. 
The architecture for the GRNN is shown in Fig 3.5 It is similar to the radial basis network with slight different second layer.

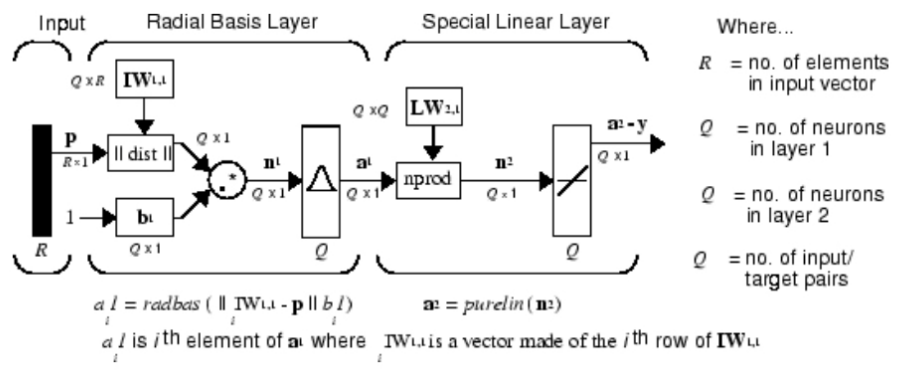

Figure 3.5: A typical structure of the GRNN in MATLAB workspace [55]

The first layer has as many neurons as there are input/ target vectors which is about 6138 in this case. It operates like the RBF. The first-layer weights are set to P', and the bias b1 is set by user to a set of numbers called SPREAD. The bigger the SPREAD is, the lower would be the number of mismatched and wrong recognitions. Each neuron's weighted input is the distance between the input vector and its weight vector, calculated with a norm, in our case, Euclidian norm. Each neuron's net input is the product of its weighted input with its bias. Each neuron's output then is calculated based on its inputs.

The second layer also has eight neurons as we assumed the number of traffic and road signs is less than 256; hence, it can be addressed by using eight binary outputs.

In order to create a GRNN, one may use the newgrnn command in Matlab. For the design of GRNN that recognizes traffic and road signs, we considered the following assumptions:

1. The traffic and road signs pictures are downloaded from the Internet. They could be in different shapes, different sizes, and different colors. The number of downloaded pictures is 58 . 
2. In order to use a unique GRNN for recognition tasks, it is required to convert all the pictures to a specific unique size and color so that the data can be fed to the network. We have to consider all of the pictures as uni-color to avoid a three-dimensional dataset instead of a two-dimensional set. Adding the third dimension for association to color does not change anything as the network could operate with two dimensional set. The resolution of the pictures is fixed to $86 * 83$. It could be set to any other numbers provided that the high-resolution pictures are available. This step is considered as data preprocessing.

3. In order to better train the network, for each sign we use the original noisefree picture plus another 19 noisy version of the picture starting from $5 \%$ noise induced to all the way to $95 \%$ noise induced to the picture in steps of 5 . In order to enrich the data set for each of these steps, we generate noisy pictures. Therefore, for each traffic or road sign, we have $2 * 19+1=39$ input-output pairs as training set. This is done for all 58 pictures. It is obvious that in our data set for all of these 39 inputs ( 1 noise-free and 38 from low to high noisy pictures), the desired output is considered to be the original picture.

4. Since we are using noise induction up to $95 \%$, it is necessary to choose the SPREAD factor as large as possible. This factor was set to 0.95 .

5. The noisy pixel is set to 255 , which represents white color code. In other words, we assume the sign pixels might be covered by the snow pieces as icy white bits. The algorithm uses four programs 1) Image_Noisy that induces a predefined amount of noise to the original picture. This program is called in Training_Data to prepare the data set, 2) Training_Data that is responsible for preparing the data set to be used in two test programs, 3) Automatic_Test in which the program is tested for all 58 pictures in Guide_Pictures directory. In this program, 
after testing the whole data with our selected amount of noise, the number of correct recognition is given at the end. If a sign is wrong, a designed wrong picture is shown as a bad result. 4) Manual_Test in which the program is tested for a chosen picture from the Guide_Picture with a predefined amount of noises. The well-known newgrnn command is used to train the network.

In order to compare the GRNN with other methods, we have also used Backpropagation neural networks, given its popularity. The simulation results show the superiority of the GRNN in comparison with Backpropagation neural networks.

As explained in this thesis we have used RBF type of neural networks, however, it is worth mentioning that other types of neural networks like Convolution Neural Network have also demonstrated very good results in pattern recognition as well. While performing very good, CNNs suffer from the volume of the computation that is a major disadvantage especially where online and real time operation is a must.

\subsection{Simulation Results}

\subsubsection{Basic Simulation Results}

In this chapter, we perform the four aforementioned programs and discuss the results. Before going more in detail, it is worth mentioning that this part is done via simulation and for the time being it is not embedded to the CANAL. In the LiDAR part, the open source code performs on-line. Let us first look at typical traffic and road signs. Fig 3.6 shows typical stop sign used in Canada. Certainly, the program could be run for other countries' traffic and road signs as well. 


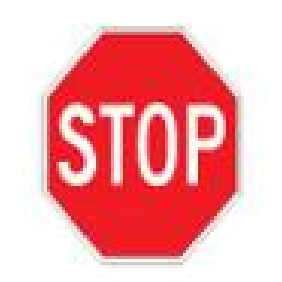

Figure 3.6: Stop sign.

This is called the original picture, which is noise-free, color, and with the original size downloaded from the Internet. Similarly, Fig 3.7 shows a road sign with its original characteristics.

While we run Automatic_Test program, all the traffic and road signs are downloaded from the Guide_Picture and stored in a data-set along with $2 * 19$ other noisy pictures. The structure of the GRNN is depicted as shown in Fig 3.8 .

As explained before, the size of the input equals 7138 ( $86^{*} 83$ number of rows and columns of each picture). The size of the input vector is $7138^{*} 1$, where the pictures are uni-color. The first hidden layer is a RBF layer with a bias value for each neuron. A set of 39 pictures is generated from the original picture, that includes 1 Original Picture plus $2 *(19$ Noisy Pictures $)=39$. This is performed for all 58 pictures, meaning that at the output of the RBF layer, a total of $2262=(39 * 58)$ neurons is required. These 2262 neurons map the intermediate data to the output, that is composed of 8 neurons in order to distinguish 256 different sign pictures. Then the percentage amount of the noise for the test stage is requested by the program as shown in

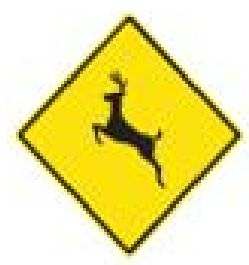

Figure 3.7: Wild animal passage area. 


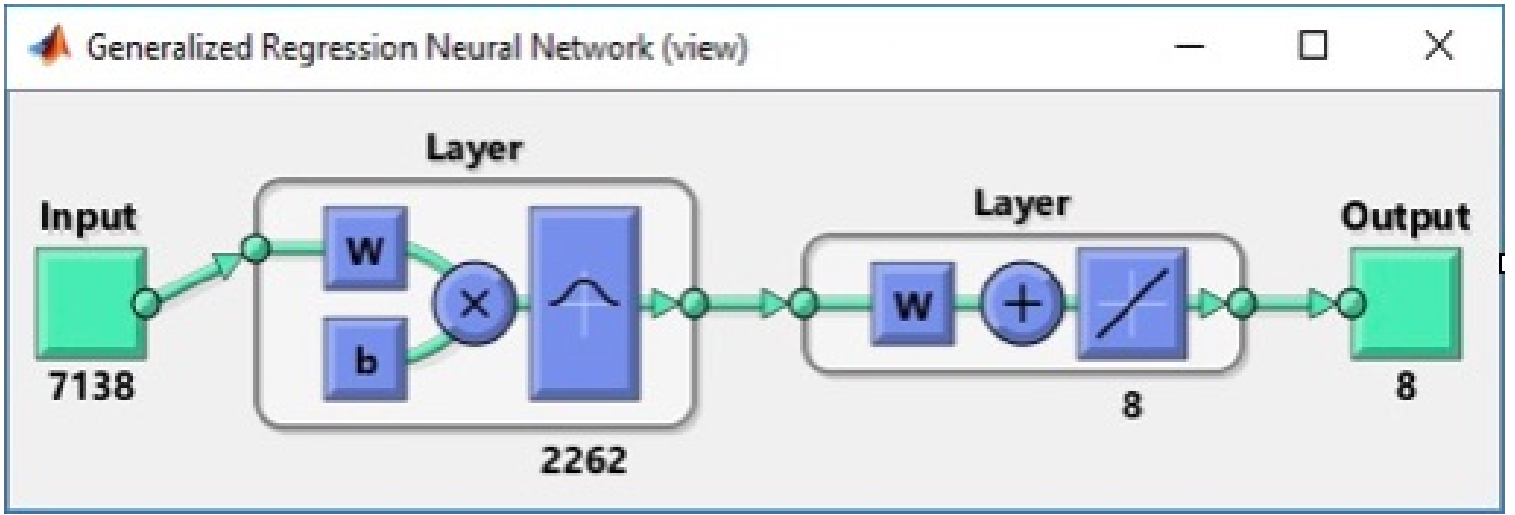

Figure 3.8: Structure of the designed GRNN.

Fig 3.9 . It is clear that the proposed method is scalable and via the aforementioned procedure, we may design different neural networks in different sizes.

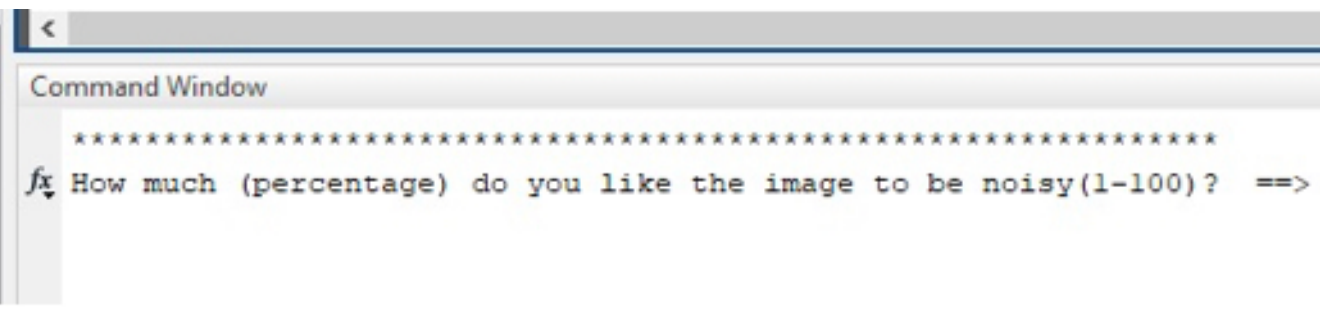

Figure 3.9: Noise percentage setup.

It is worth noting that regardless of the amount which is entered in this step, the GRNN has already seen a basket of noisy pictures in order to complete its training phase. After entering the amount of the noise, the program is run for all 58 pictures and indicates if the recognition is correct or not. For example, by selecting the percentage amount of noise 50 , then all the 58 pictures become noisy. In other words, $50 \%$ of the 6138 pixels are set to 255 (white color) randomly (random position of the pixels without repeat).

Fig 3.10 and Fig 3.11 shows both the noisy picture and the recognized sign by the neural network. 


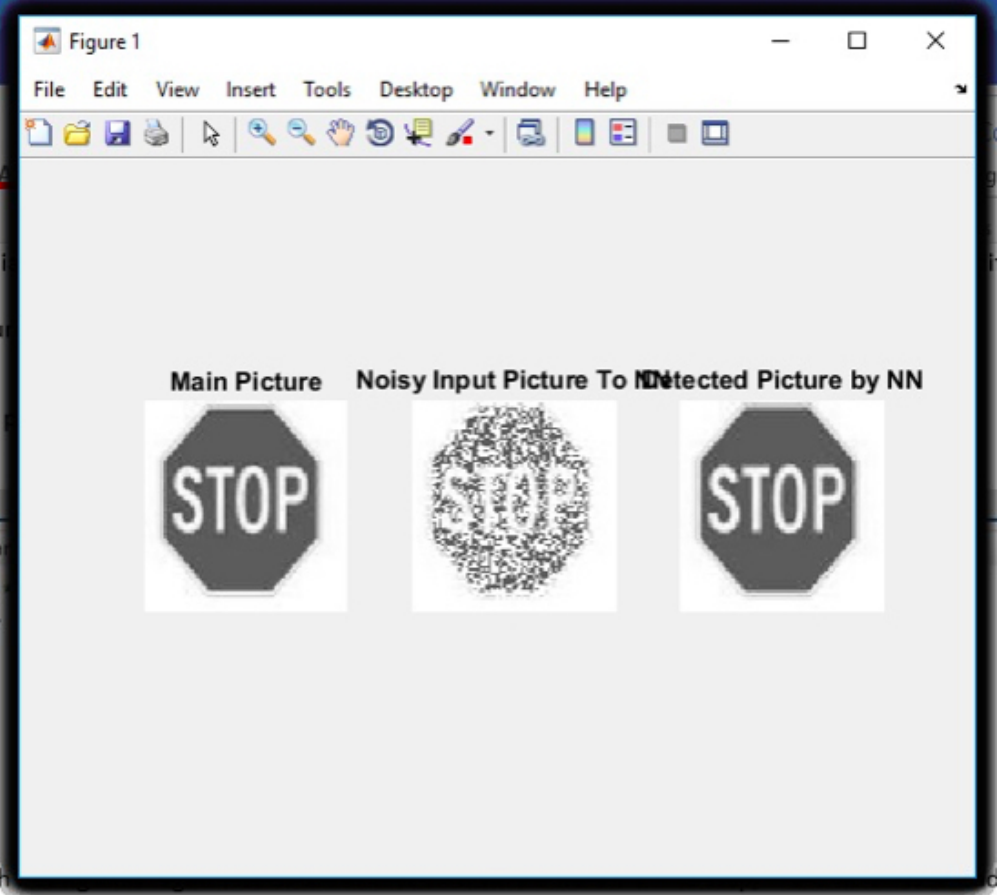

Figure 3.10: Simulation result for $50 \%$ noisy stop sign.

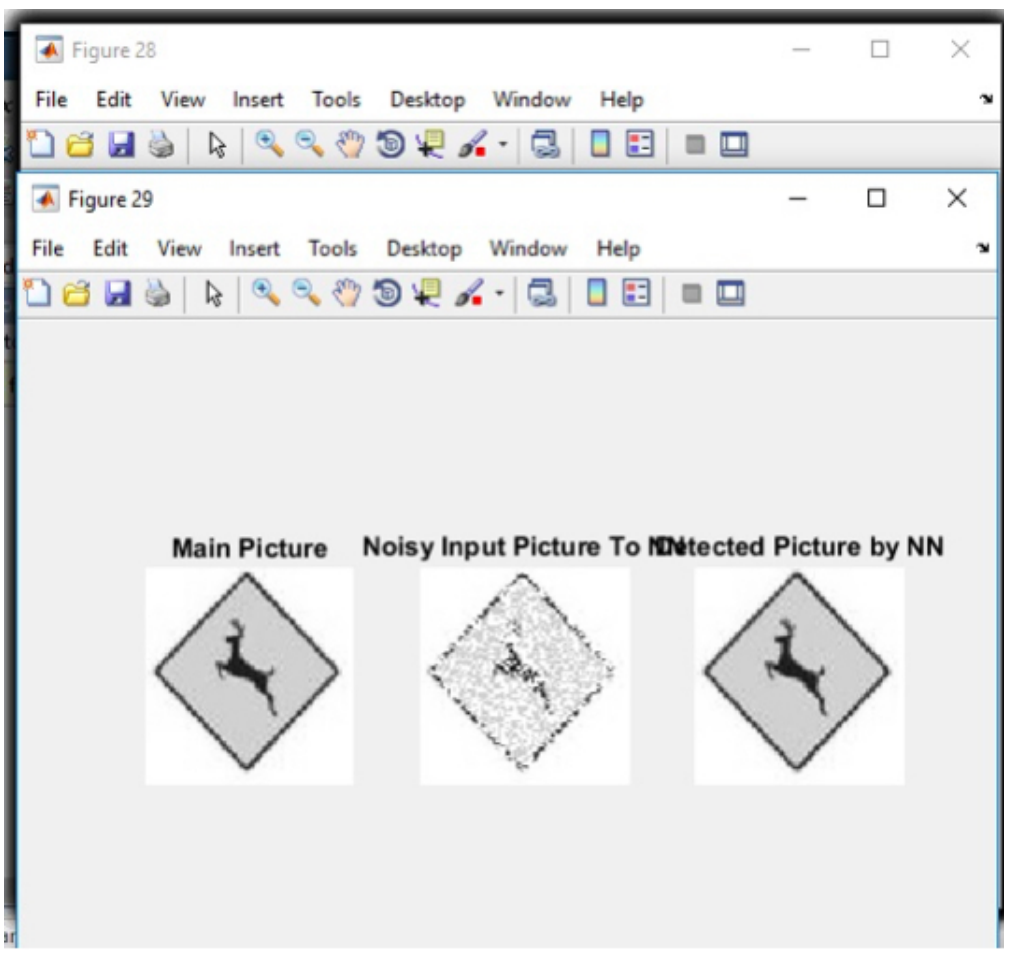

Figure 3.11: Simulation result for 50\% noisy wild animal area. 
In both figures, we can see the original signs at the left, $50 \%$ noisy signs in the middle, and the recognized result at the right. The performance is $100 \%$ good as shown in Fig. 3.12 .

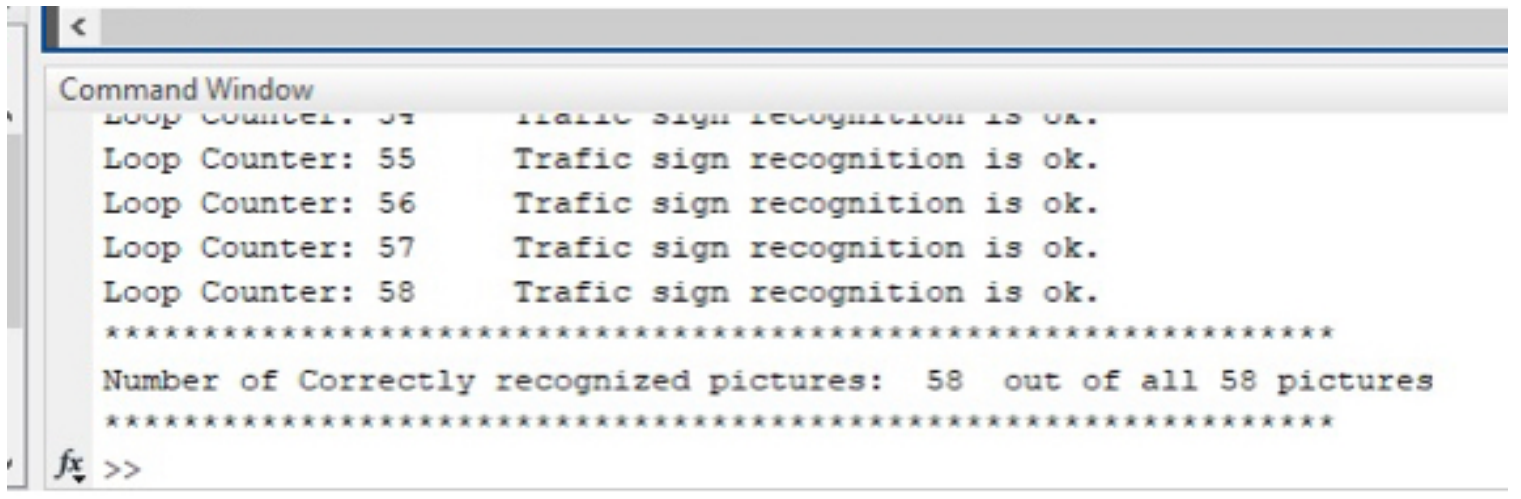

Figure 3.12: The performance of the proposed structure 58 out of 58 .

We also considered three other traffic and road signs with $35 \%, 65 \%$, and $95 \%$ induced noise. As depicted below, they are all recognized correctly. First, we select P_24 which is School Bus Attention Sign with 35\% induced noise. Running Manual_Test program, the result is shown in Fig. 3.13 .

As it is seen, the program has recognized the picture correctly. Now, we select a miniature picture (P_18), which is No Bicycle Sign with $65 \%$ of induced noise. Again as depicted in Fig 5.2 , the GRNN was able to recognize this picture correctly.

And finally, we select No Parking Sign with $85 \%$ of induced noise. The simulation result is shown in Fig.3.15. As shown in the picture, recognition of such a noisy picture is even very hard for human-being while the network has recognized it correctly.

Clearly, the proposed network is able to recognize noisy patterns. It is basically a part of the smart system which is employed in an autonomous vehicle for a smart drive. We need some extra soft-hardware parts to capture the data from the environment, 


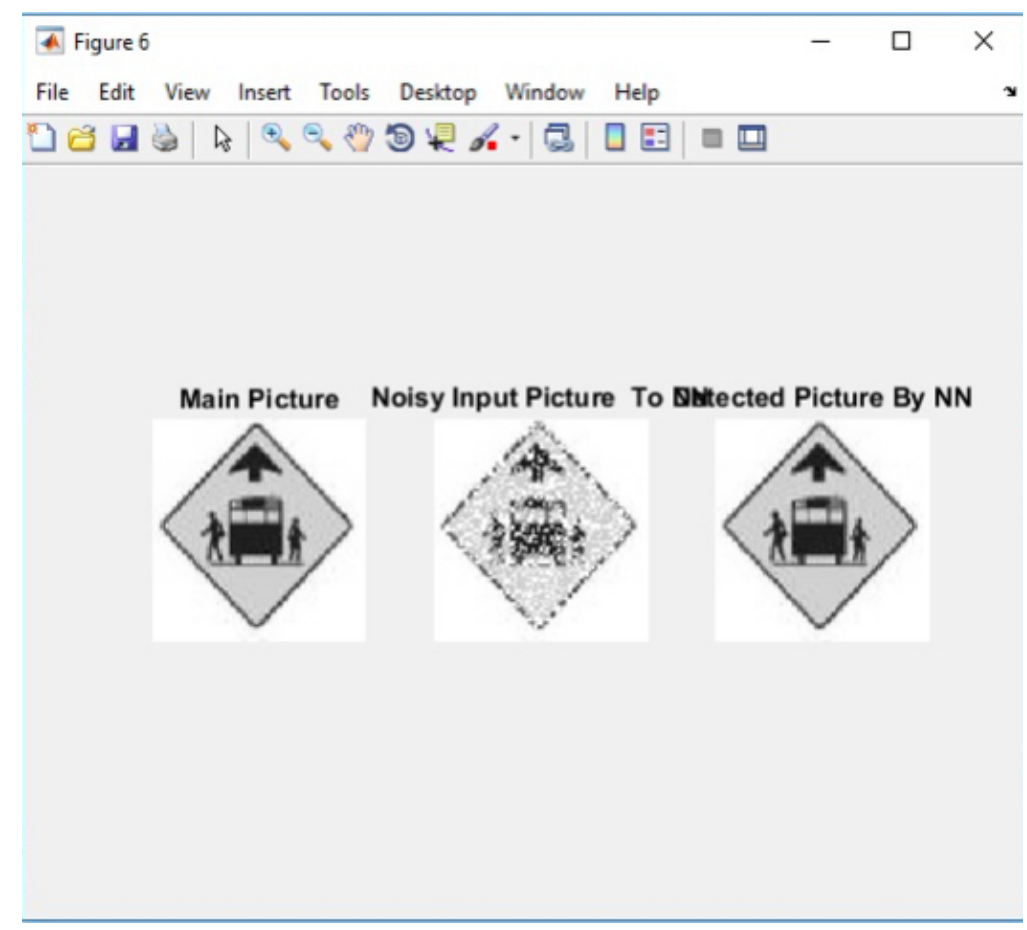

Figure 3.13: School Bus Attention Sign with 35\% of induced noise.

tag on the road and traffic signs, cut the signs part from the whole picture, and send it to the pattern recognition subsystem which will be discussed in subsection 3.6.3 shortly.

As shown in the simulation results and as the result of many times simulation, it is clear that the proposed method is reliable as much as other neural network based methods are and even better. Moreover, although it takes a little bit of time when the network is being trained, the network performs very quickly while testing the new noisy patterns.

\subsubsection{Comparison}

In order to compare the performance of the proposed RBF-based neural network, another popular neural network-based method is considered. Backpropagation neural networks have shown very promising results in many applications. Therefore, as a 


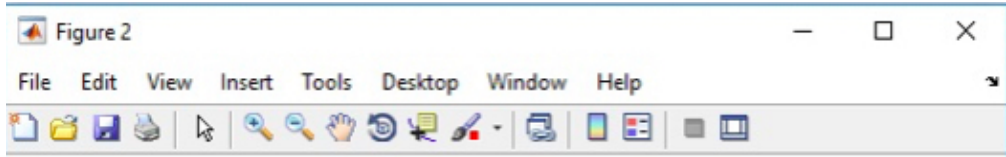
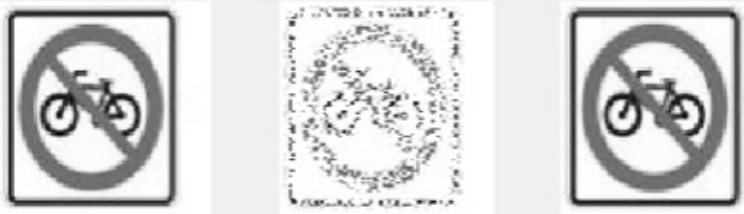

Figure 3.14: No Bicycle Sign with $65 \%$ of induced noise.

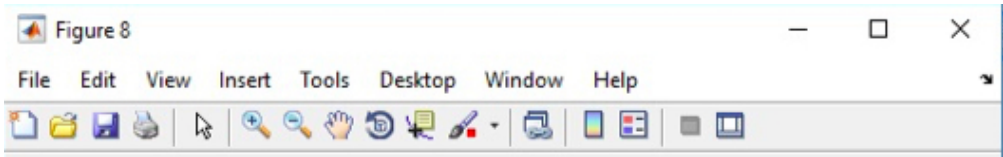

Main Picture Noisy Input Picture To Ditected Picture By NN

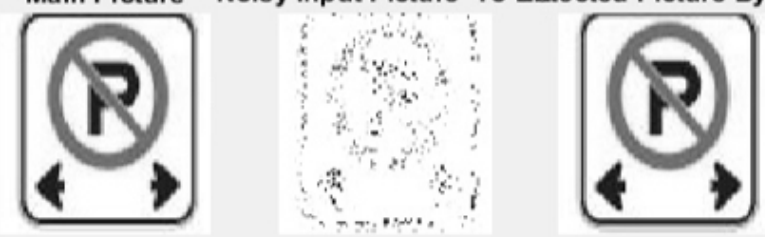

Figure 3.15: No Parking Sign with 85\% of induced noise. 
regular practice, a BP-based neural network is designed and set-up, as shown in Fig. 3.16 .

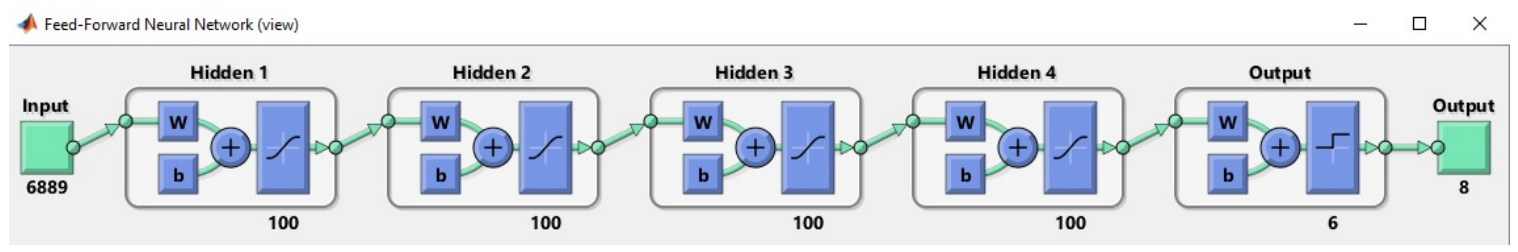

Figure 3.16: A five-layer backpropagation neural network with enough neurons in each layer.

As shown in the figure, the BP-based neural network is a rich one in terms of the number of layers and the number of neurons in each layer. Fig.3.17) shows the process of learning in the proposed BP-based neural network.

There are two important findings from the simulation. One is that it takes too much time for the network to learn and be able to recognize a pattern. This is not practical as real-life use cases are real-time. The other finding is that it cannot recognize the traffic and road signs even when there is only $50 \%$ of noise. Fig. 3.18 shows that none of the 59 signs has been recognized correctly.

In such a case, the program outputs a symbolic result as shown in Fig. 3.19 .

By decreasing the level of noise (with trial and error), it is observed that with noise level of only $25 \%$, only one of 58 signs is recognized correctly. Fig. 3.20 shows the simulation result. The sign which has been recognized correctly among all 58 sign is shown in Fig. 3.21

In summary, the proposed method could recognize the pictures even when they are subjected up to $90 \%$ noise. As a result, the RBF-based neural network is a suitable 


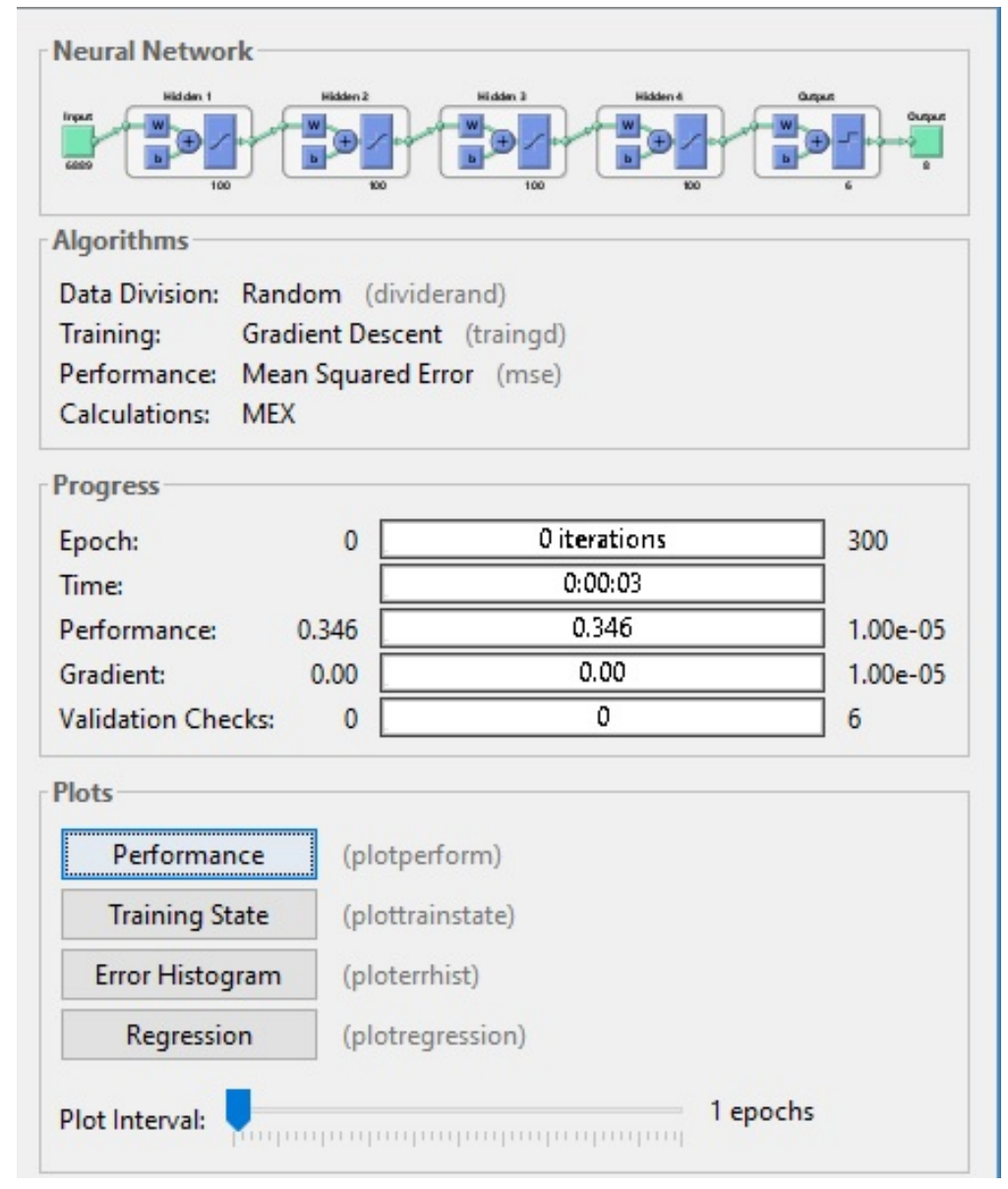

Figure 3.17: Learning process in the proposed neural network.

network for traffic sign recognition in autonomous cars.

To compare the achieved results in this thesis with the published works in the literature, we examine some of the state of the art research, although a very comprehensive survey in the field has been published by Safat et al [56].

M. Badrul has published a paper that is the result of a thesis done in the field of traffic and road sign recognition in Bangladesh [57]. The developed system is based on the data gathered from a camera, and the recognition is performed by using a neural network. A feature detection strategy has been used as well at the beginning of the process. This part is somehow sign dependent. In other words, if the signs are changed, then this part has to be customized accordingly. According to the results 


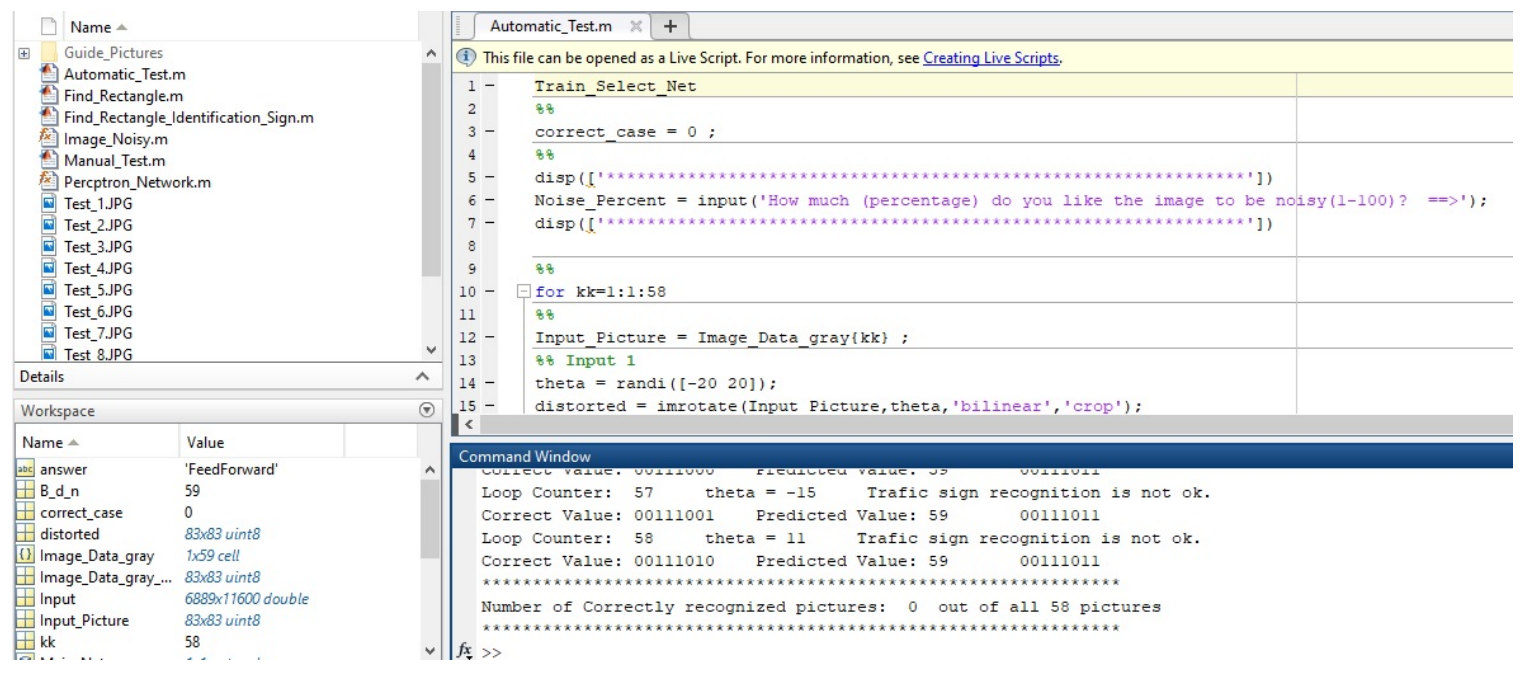

Figure 3.18: 0 out of all 58 pictures is recognized correctly!

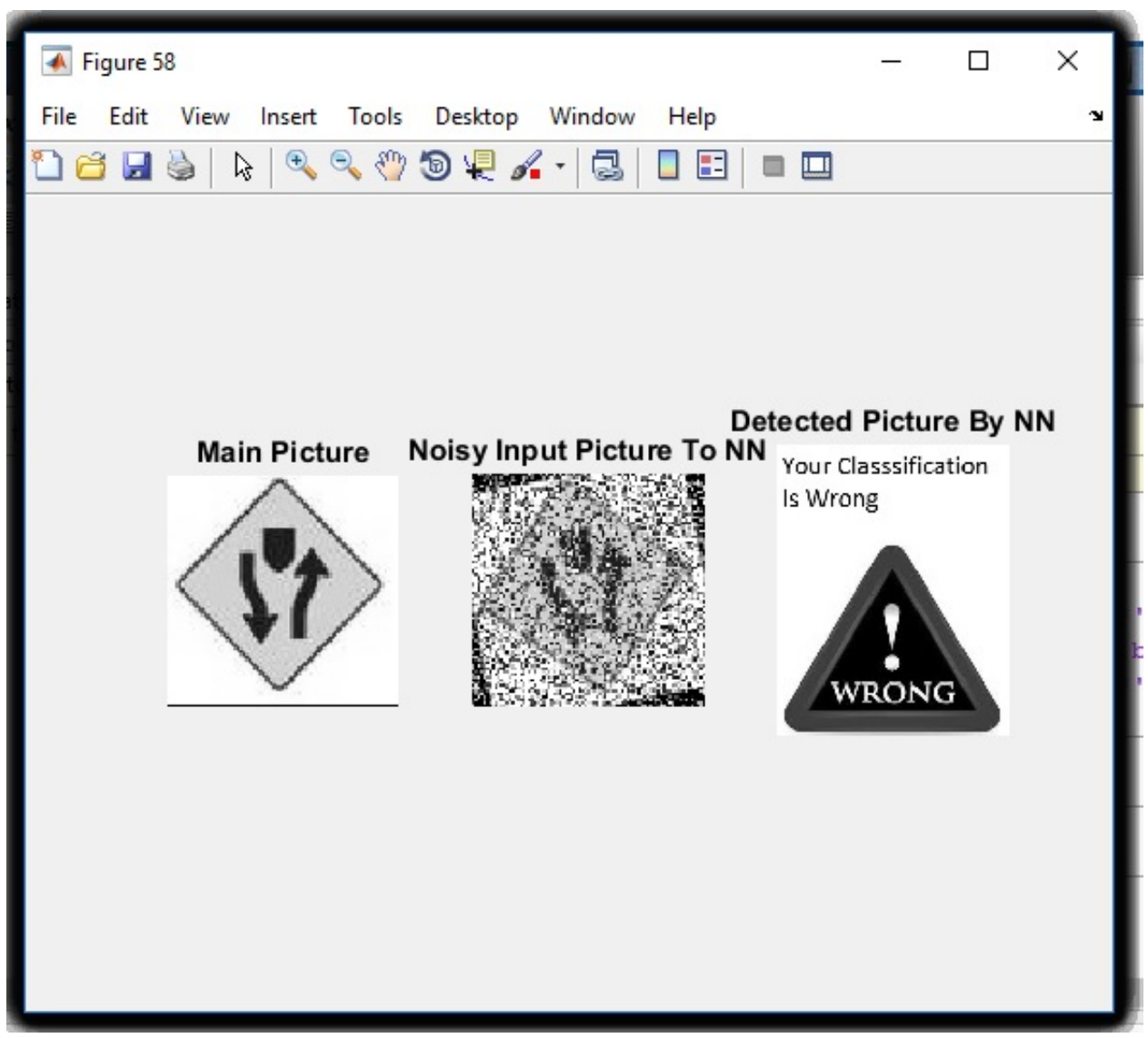

Figure 3.19: Wrong classification while using BP-based neural network subject to $50 \%$ added noise. 


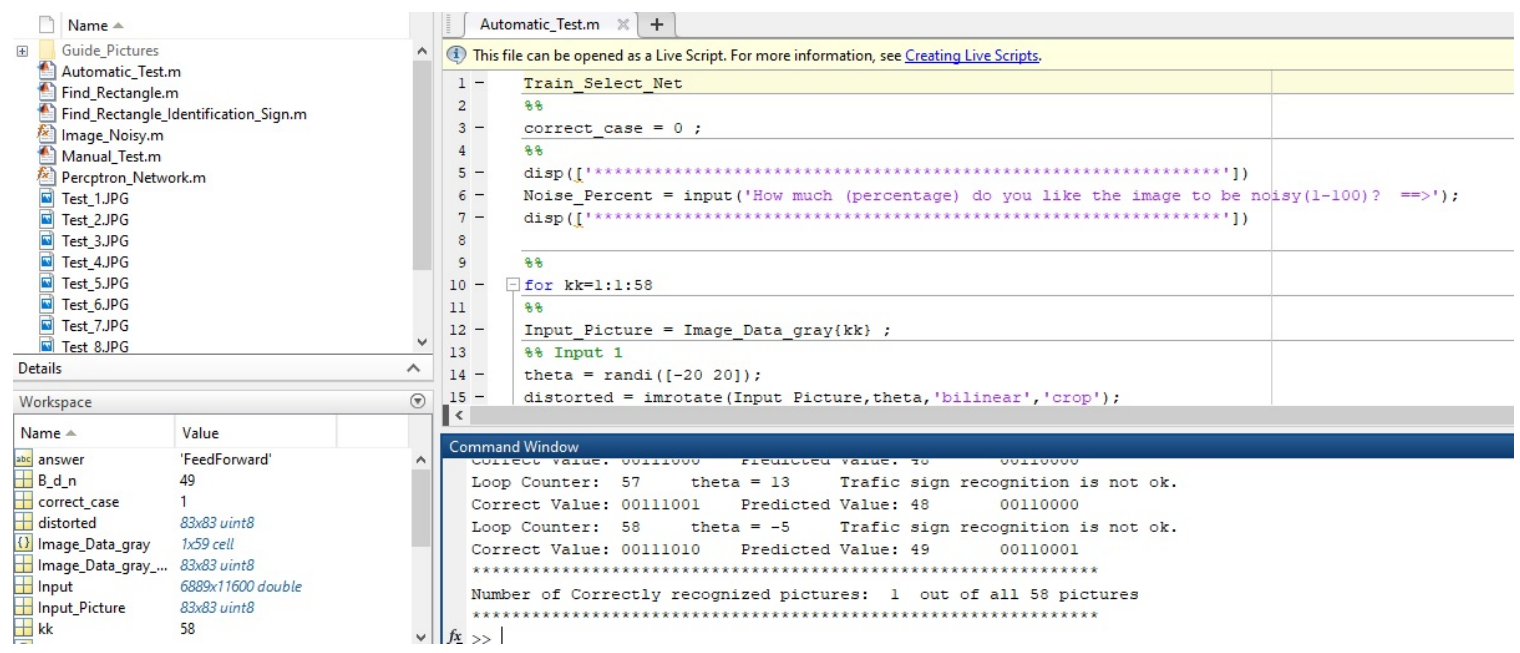

Figure 3.20: 1 out of all 58 pictures is recognized correctly where the level of noise is $25 \%$ !

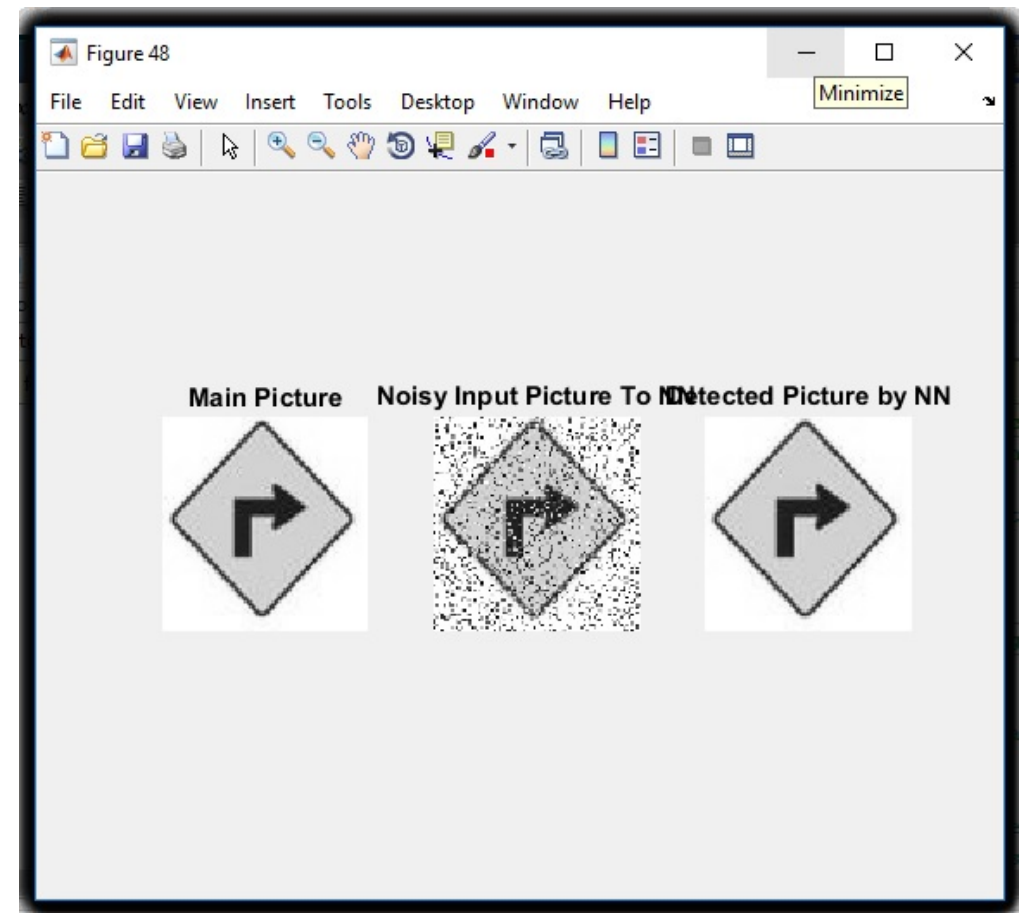

Figure 3.21: The only correctly recognized picture by using BP-based neural network where the level of noise is $25 \%$.

presented by the paper, the success rate is only $91.5 \%$, whereas, in our proposed GRNN, the success rate is $100 \%$ (58 out of all 58 signs). Moreover, although it is not highlighted exactly how much noise has been involved in the simulation results 
of [57]. By inspecting Fig 3.22 copied from the reference [57] as the noisy sign, it is clear that the noise level is much less than $90 \%$, a level that was successfully tested.

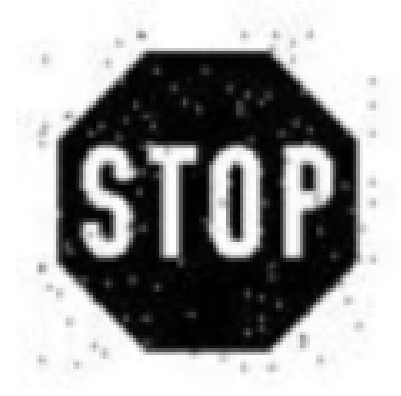

Figure 3.22: The noisy sign of the reference [57].

It is also worth mentioning that in our proposed method, there is no difference between all 58 signs. In other words, as soon as the network is trained, it recognizes all the signs correctly, while in [57, the network is customized for only mandatory four signs. The paper, however, claims that it is performing better than other reference papers.

Sabrine Hamdiet et al has proposed a neural network-based approach for sign classification [58]. The proposed network is composed of two neural networks. The first neural network recognizes the shape of the sign as it is classifies the input sign into a triangle, rectangle, or circle. The second network recognizes the sign itself. The success rate for shape recognition varies from $90 \%$ up to the $100 \%$ depending on the shape with the average rate of $94.5 \%$. The success rate for sign recognition varies from $80 \%$ up to $90 \%$, with an average of $89 \%$. Since these two networks are in series, the success rate, statistically, is $94.5 \% * 89 \%=84.1 \%$. It is worth noting that this work considers almost all traffic signs, which is an advantage. However, all signs are noise-free. In contrast, our method can recognize the signs with the noise as much as $90 \%$. 
The same idea has been employed by K. Tohidul and R. G. Raj [59]. The paper claims that up to $99.9 \%$ accuracy is achievable for Malaysian traffic signs. As shown in this work, a high level of accuracy is achieved even for a noisy sign when GRNN is employed. This is counted as a promising result, although the test is performed where the signs are not subject to noise which is different from our test condition where up to $90 \%$ of noises are added to the picture.

There have been extensive researches on Convolutional Neural Networks (CNNs) in recent years. Like any other method, CNN has its own advantages and disadvantages. The main disadvantage of the CNN is the large number of layers and neurons that is required to perform classification. Therefore, CNN may not be a promising candidate for real-time applications. But in terms of accuracy, CNN usually offers a good performance.

A two-stage algorithm has proposed for German and Chinese traffic sign recognition by Famin Shao et al [60]. In the first stage, considered as the preprocessing phase, a Wavelets algorithm is used to prepare the data, and in the second stage, a CNN is employed for sign recognition. The data set is very rich, and the authors have achieved very good results. The only difference between their method and the method used in this thesis is the noise. The signs in [60] are noise-free while we have considered a noise level up to $90 \%$.

R-CNN is a new convolutional neural network usually used for object detection. It has a lot of computational burdens. J. Li and Z. Wang has proposed a R-CNN for traffic sign recognition [61]. Their proposed method is capable of recognizing the signs with $99.4 \%$ accuracy, yet, with the assumption of noise-free signs.

In order to better compare our result with other methods in the literature, some indexes are introduced based on which the methods are evaluated.

The following table compares different methods based on some criteria, namely, 
percentage of error, noise, being subject to noise and its percentage, real life cases like rotation and recognizing the sign in a whole picture.

\begin{tabular}{|c|c|c|c|c|c|c|}
\hline Reference & Method & $\begin{array}{l}\text { Being } \\
\text { subject to } \\
\text { noise(\%) }\end{array}$ & $\begin{array}{l}\text { Percentage } \\
\text { of success }\end{array}$ & $\begin{array}{l}\text { Real life } \\
\text { applica- } \\
\text { tion }\end{array}$ & $\begin{array}{l}\text { Real life } \\
\text { applica- } \\
\text { tions and } \\
\text { subject to } \\
\text { noise }(\%)\end{array}$ & $\begin{array}{l}\text { Percentage } \\
\text { of success }\end{array}$ \\
\hline $\begin{array}{l}{[56] \text { Sur- }} \\
\text { vey paper }\end{array}$ & ANN & No & $99.46 \%$ & No & $\begin{array}{l}\text { Not appli- } \\
\text { cable }\end{array}$ & $\begin{array}{l}\text { Not appli- } \\
\text { cable }\end{array}$ \\
\hline $\begin{array}{l}{[56] \text { Sur- }} \\
\text { vey paper }\end{array}$ & $\begin{array}{l}\text { Deep } \\
\text { learning }\end{array}$ & No & $98.6 \%$ & No & $\begin{array}{l}\text { Not appli- } \\
\text { cable }\end{array}$ & $\begin{array}{l}\text { Not appli- } \\
\text { cable }\end{array}$ \\
\hline$[57]$ & $\begin{array}{l}\text { Feature } \\
\text { detec- } \\
\text { tion(NN), } \\
\text { sign de- } \\
\text { pendent }\end{array}$ & $\begin{array}{l}\text { Yes(estimat } \\
\text { less than } \\
25 \%)\end{array}$ & (61.5\% & No & $\begin{array}{l}\text { Not appli- } \\
\text { cable }\end{array}$ & $\begin{array}{l}\text { Not appli- } \\
\text { cable }\end{array}$ \\
\hline [58] & $\begin{array}{l}\text { ANN(two } \\
\text { NN) }\end{array}$ & No & $\begin{array}{l}84.1 \% \\
\text { overall }\end{array}$ & No & $\begin{array}{l}\text { Not appli- } \\
\text { cable }\end{array}$ & $\begin{array}{l}\text { Not appli- } \\
\text { cable }\end{array}$ \\
\hline [59] & ANN & No & $99.9 \%$ & No & $\begin{array}{l}\text { Not appli- } \\
\text { cable }\end{array}$ & $\begin{array}{l}\text { Not appli- } \\
\text { cable }\end{array}$ \\
\hline [60] & $\begin{array}{l}\text { Wavelet + } \\
\text { CNN }\end{array}$ & No & $\begin{array}{l}\text { Not re- } \\
\text { ported but } \\
\text { looks good }\end{array}$ & No & $\begin{array}{l}\text { Not appli- } \\
\text { cable }\end{array}$ & $\begin{array}{l}\text { Not appli- } \\
\text { cable }\end{array}$ \\
\hline [61] & R-CNN & No & $99.4 \%$ & No & $\begin{array}{l}\text { Not appli- } \\
\text { cable }\end{array}$ & $\begin{array}{l}\text { Not appli- } \\
\text { cable }\end{array}$ \\
\hline This thesis & BPNN & Yes(25\%) & $\begin{array}{l}1 \text { out of } 58 \\
\text { sign recog- } \\
\text { nized cor- } \\
\text { rectly }\end{array}$ & No & $\begin{array}{l}\text { Not appli- } \\
\text { cable }\end{array}$ & $\begin{array}{l}\text { Not appli- } \\
\text { cable }\end{array}$ \\
\hline This thesis & RBF & $\begin{array}{l}\text { Yes(up to } \\
95 \%)\end{array}$ & $100 \%$ & Yes & $\begin{array}{l}\text { Up to } 50 \% \\
\text { of noise } \\
\text { and +- } \\
25 \text { degree } \\
\text { rotation }\end{array}$ & $100 \%$ \\
\hline
\end{tabular}

Table 3.1: A comparison between features and percentage of errors in different methods. 


\subsubsection{Real Life Situation Scenarios}

In real-life applications, the roads are not leveled exactly; therefore, the x-axis of the vehicle and installed camera on the vehicle is not exactly in parallel with the $\mathrm{x}$-axis of the traffic and road signs. This is also the case when and where the vehicle is passing a puddle on the road. In other words, in such cases that are popular in a normal ride, there is a delta angle between the axis of the car and axis of the sign while the network is trained based on the data with no delta angle. To make sure that the network is capable of recognizing the sign where the delta angle is not zero, we need to train the network with a new data set. The program is modified, and the network is trained, and the simulation results are presented here. As explained in the previous section, the GRNN based on the RBF neural networks outperforms others. A two-layer neural network, namely a RBF and a Perceptron output layer, shown in Fig 3.23 is trained with a new data set with rotated pictures. The level of noise is set to $50 \%$, although we may try for up to $80 \%$ of noise as well.

The program is run and, as shown in Fig 3.24 and Fig 3.25 , all of the examined 58 pictures are tested, and the GRNN recognizes all 58 correctly.

In the following Figs. 3.26 to 3.33 , we see some simulation results for different 


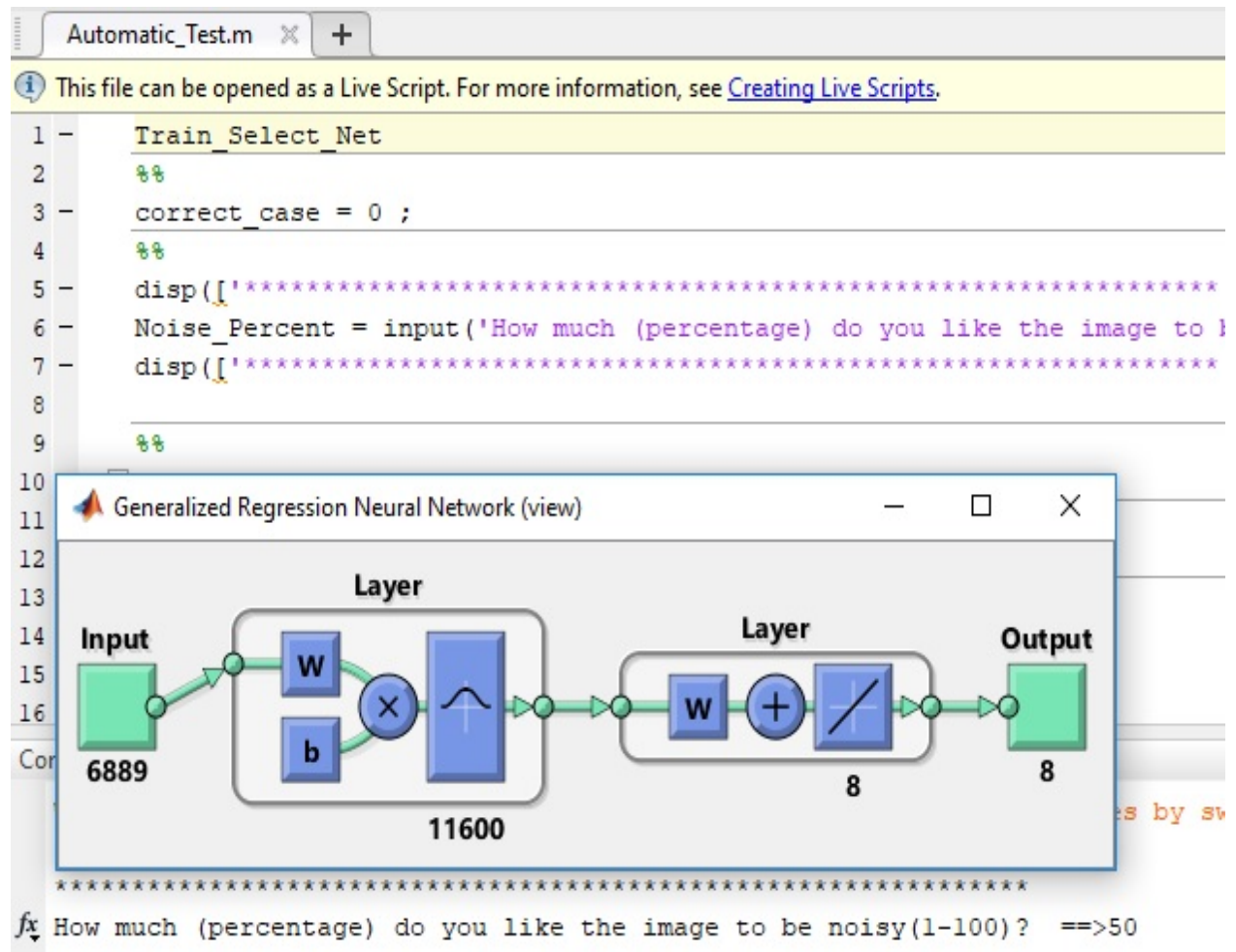

Figure 3.23: A two-layer RBF neural network.

traffic and road signs.

The real-life applications and situations are still much complicated than a noisy and rotated sign recognition. To consider more complicated situations, we train the network to recognize the signs in a rectangle. The data set is shown in Fig. 3.34 and Fig 3.35. Now the trained network is used to recognize the traffic sign in a real-life 


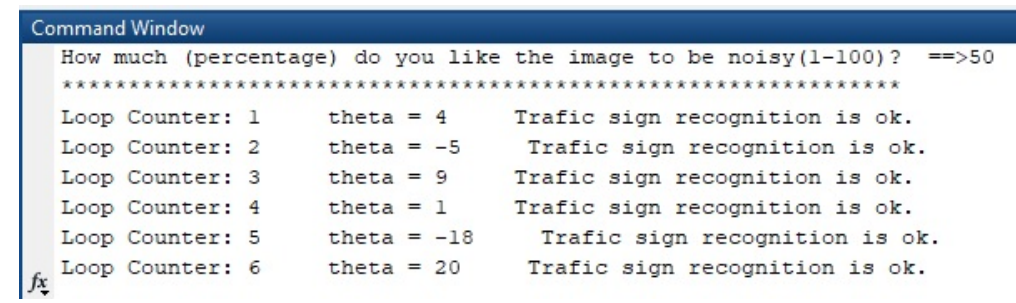

Figure 3.24: A two-layer RBF neural network.

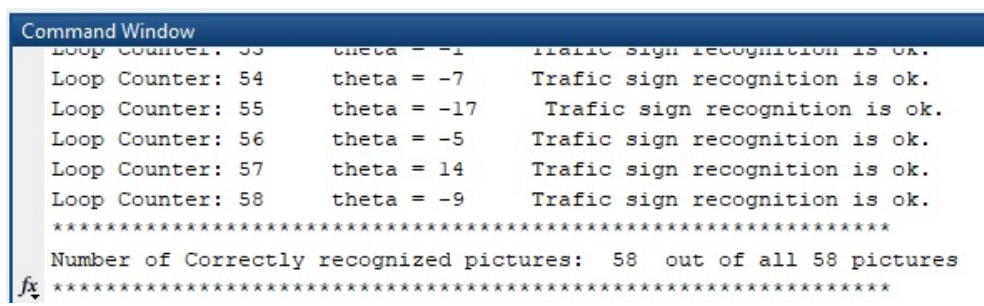

Figure 3.25: A two-layer RBF neural network.

picture. An example of real-life picture is shown in Fig.3.36. The converted gray picture is shown in the following Fig 3.37. Having applied the modified program and the trained network on the above picture, the network can recognize the sign most of the time. The result is shown in the following Fig. 3.38 .

It is worth noting that the trained network is not capable of recognizing the sign all the time. For example, after a couple of tries, the network recognizes the sign incorrectly as the following Fig 3.39 .

More modification is required as the future research work of this thesis. 


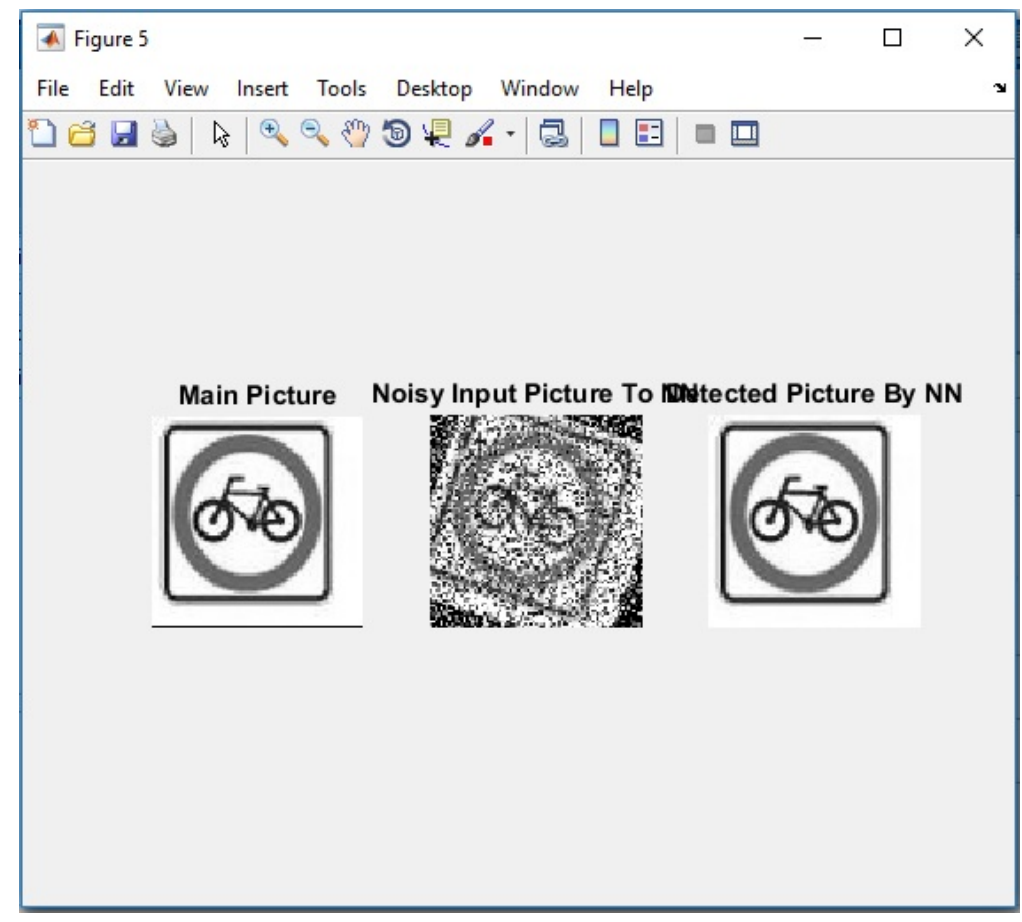

Figure 3.26: Bicycle sign where it is -18 degree rotated with $50 \%$ noise.

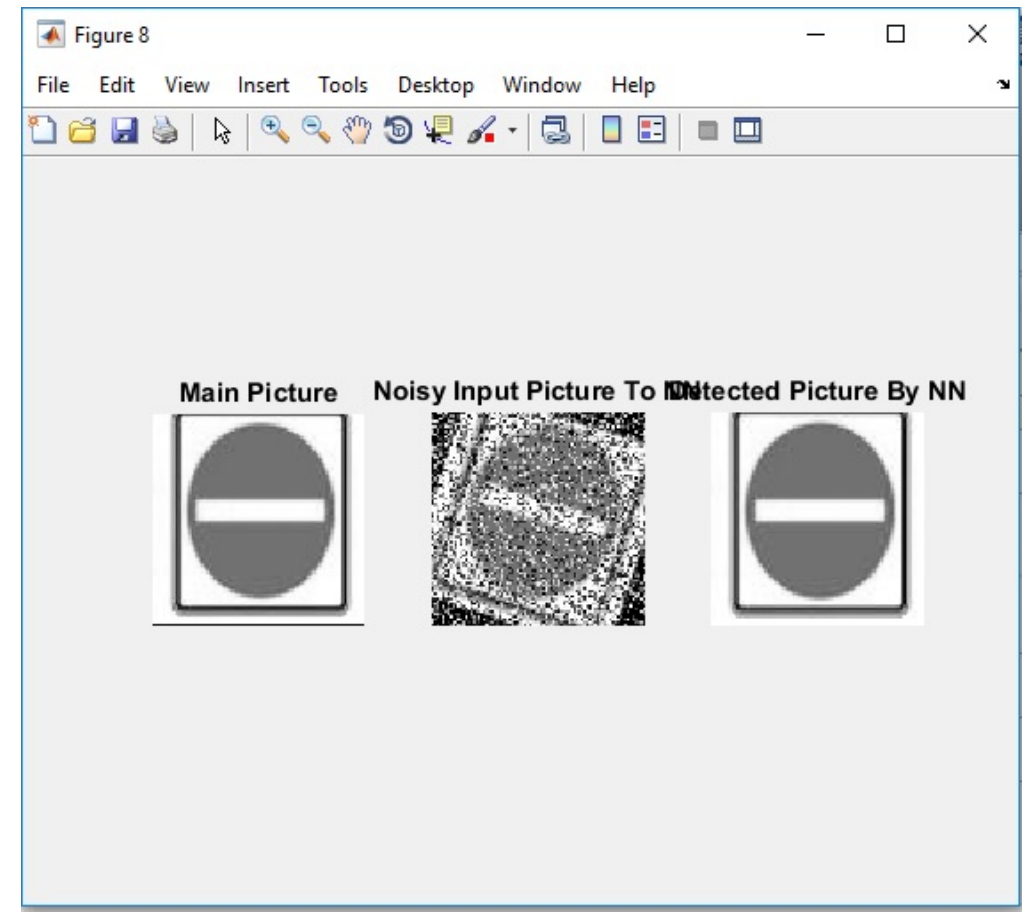

Figure 3.27: Entry forbidden sign where it is -20 degree rotated with $50 \%$ noise. 


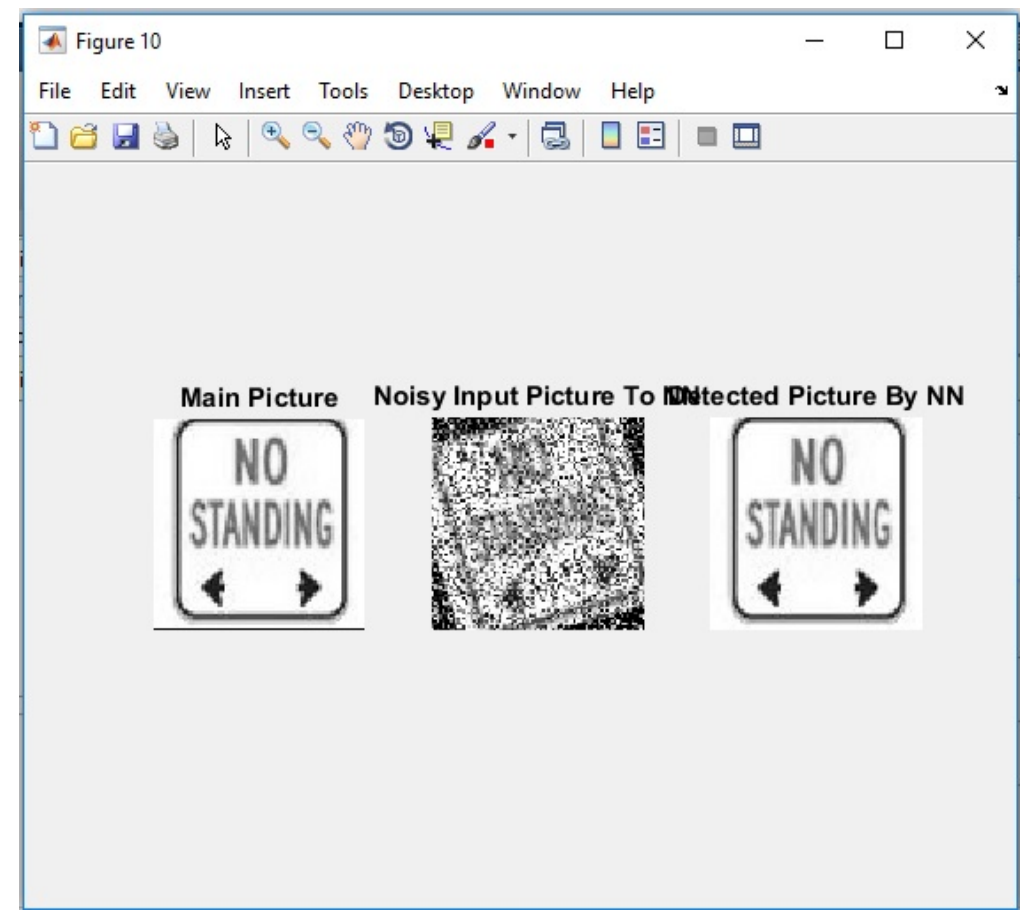

Figure 3.28: No standing sign where it is - 18 degree rotated with $50 \%$ noise.

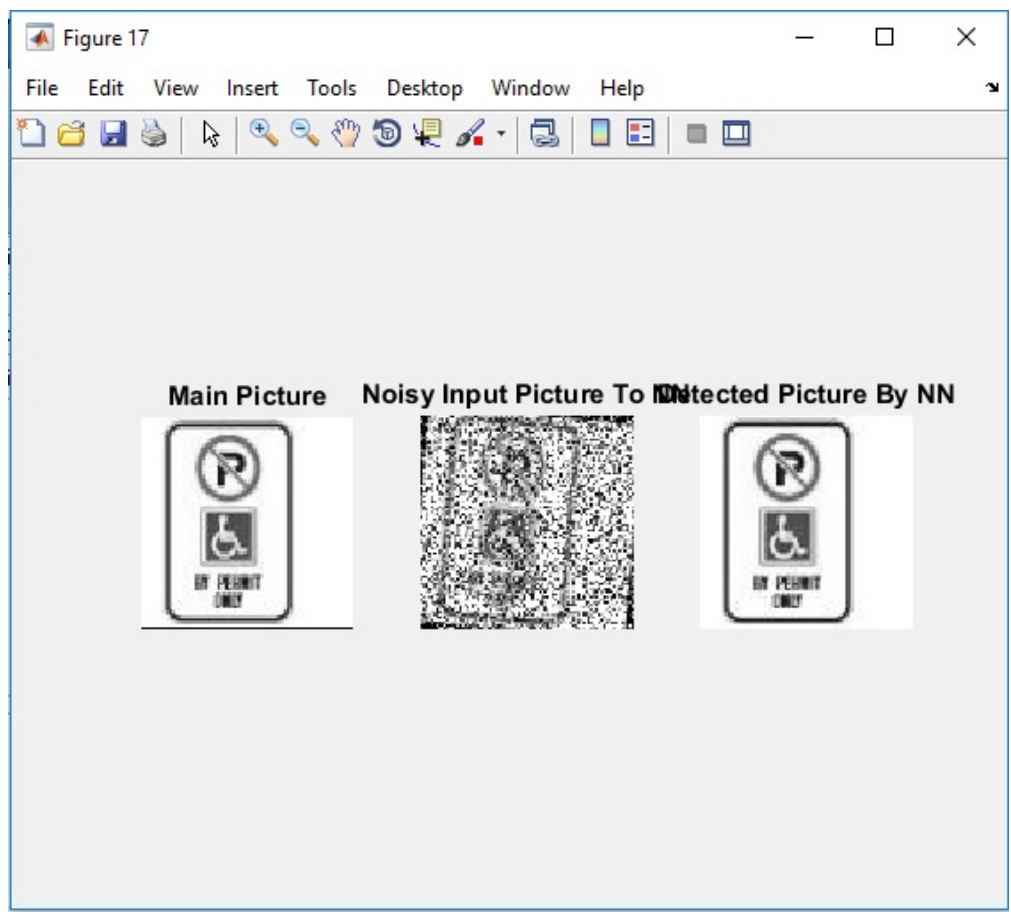

Figure 3.29: Handicap parking sign where it is +5 degree rotated with $50 \%$ noise. 


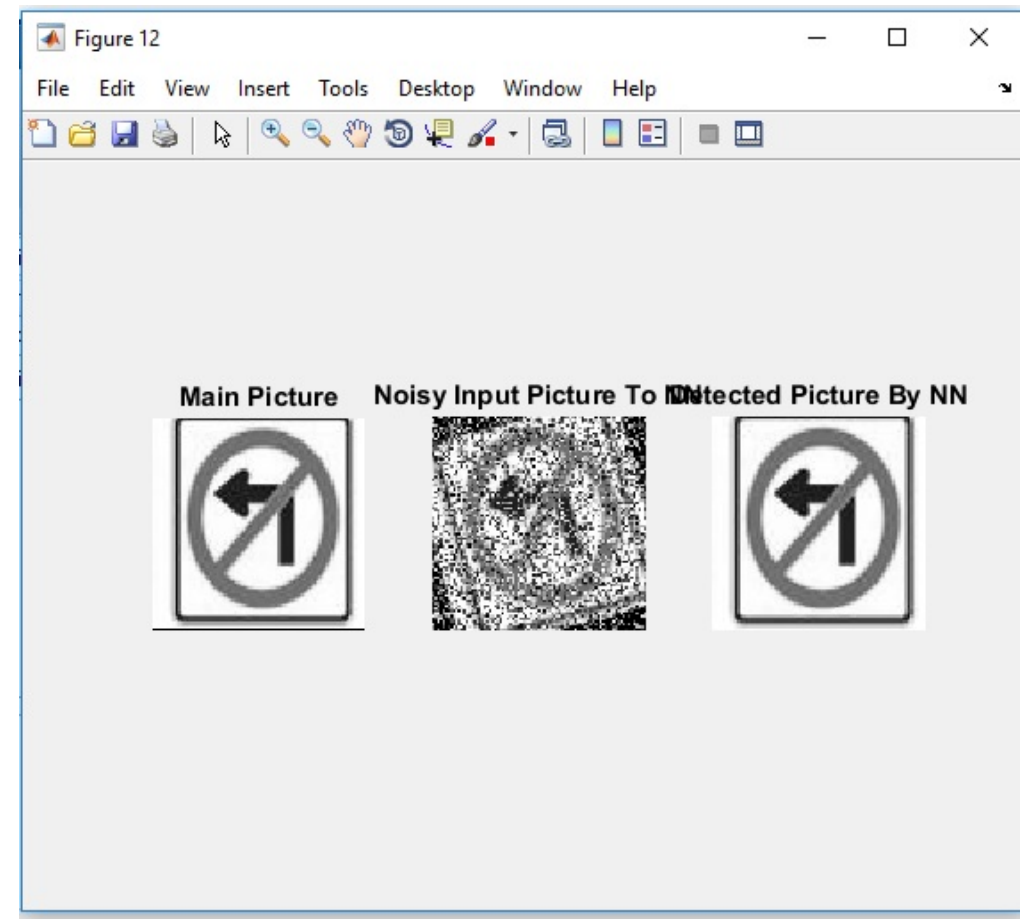

Figure 3.30: Turning left forbidden sign where it is +15 degree rotated with $50 \%$ noise.

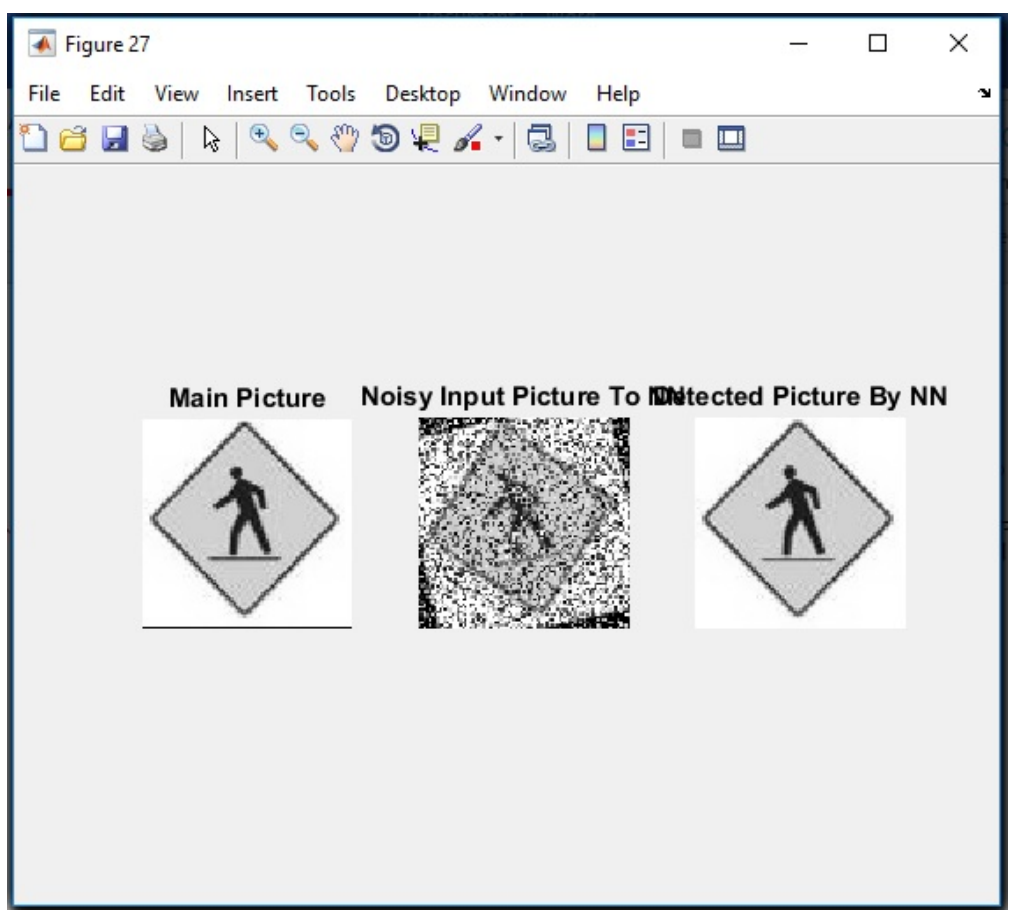

Figure 3.31: Pedestrian sign where it is - 12 degree rotated with $50 \%$ noise. 


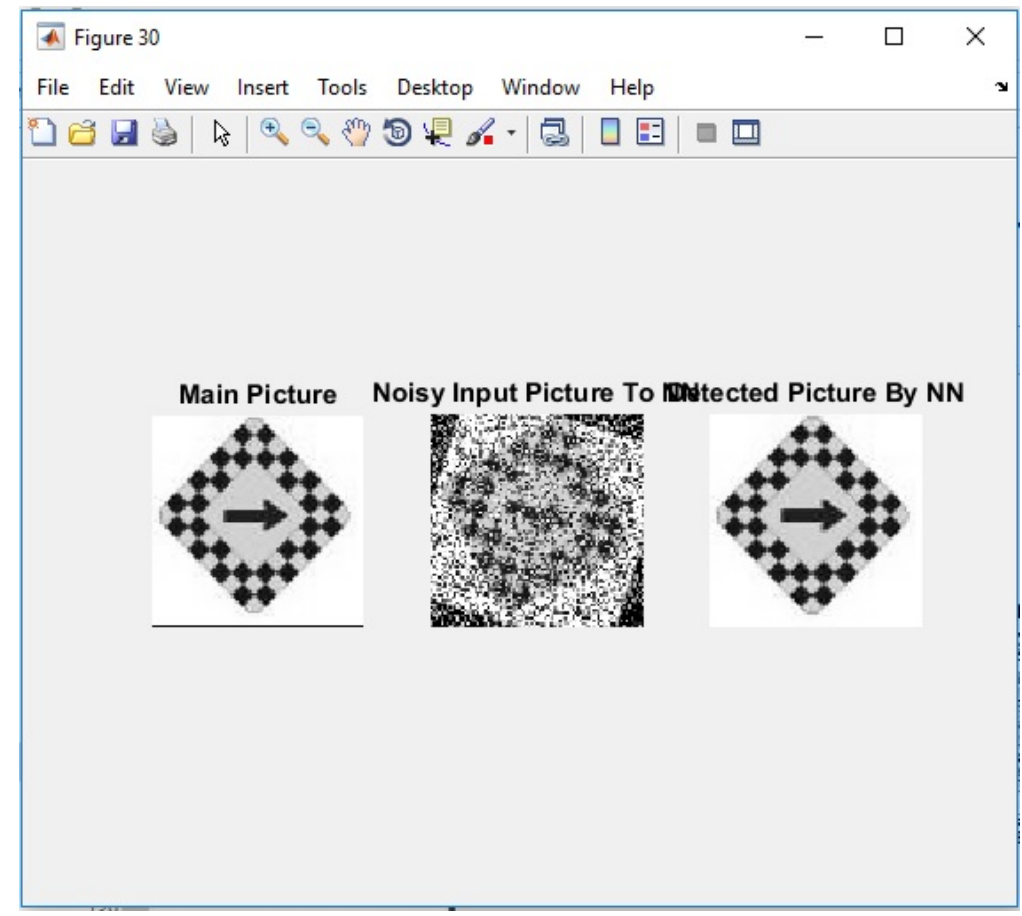

Figure 3.32: A road sign where it is -17 degree rotated with $50 \%$ noise.

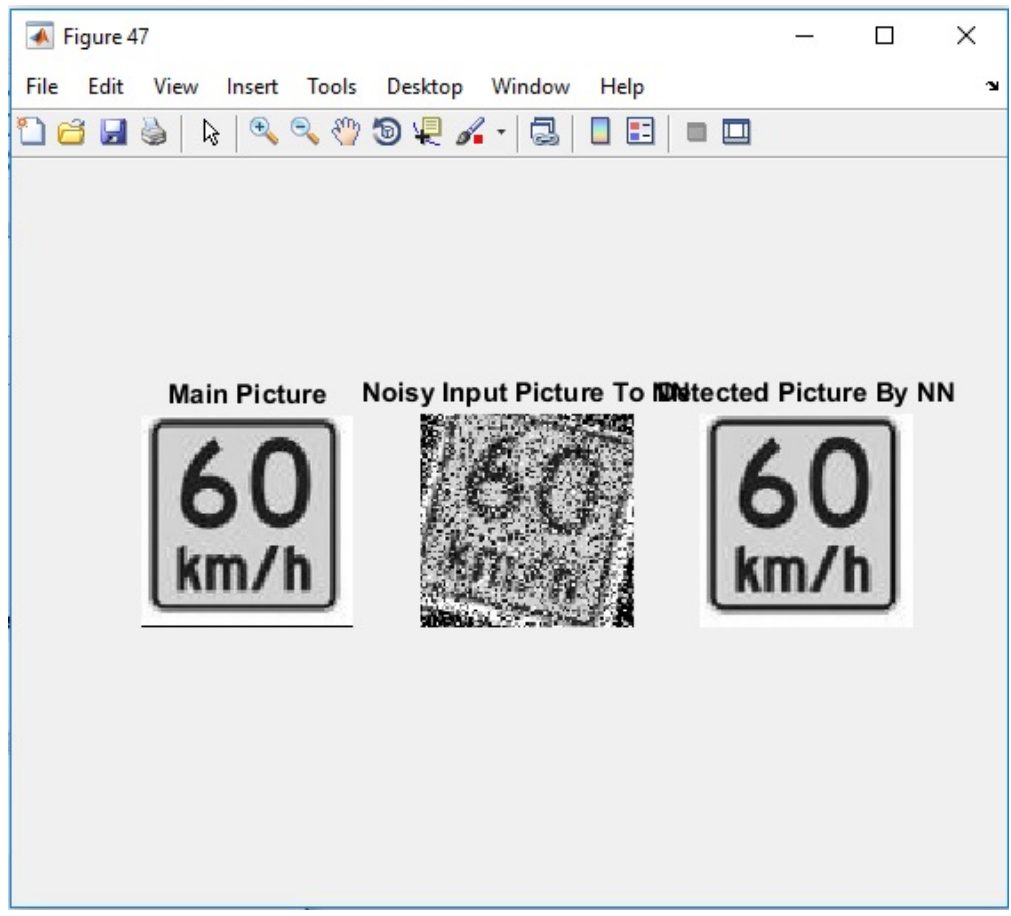

Figure 3.33: Speed sign where it is -13 degree rotated with $50 \%$ noise. 

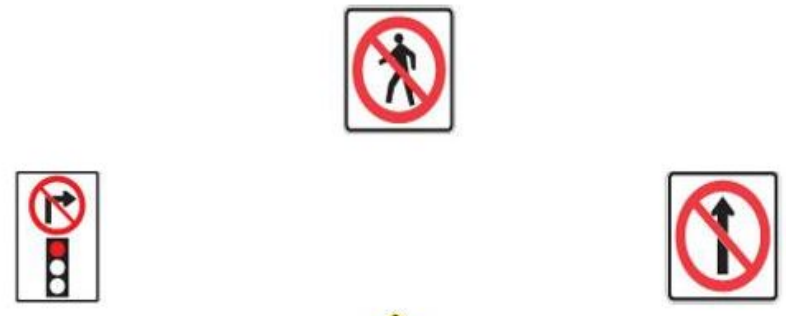

Figure 3.34: The data set is used to train the network for real life applications.
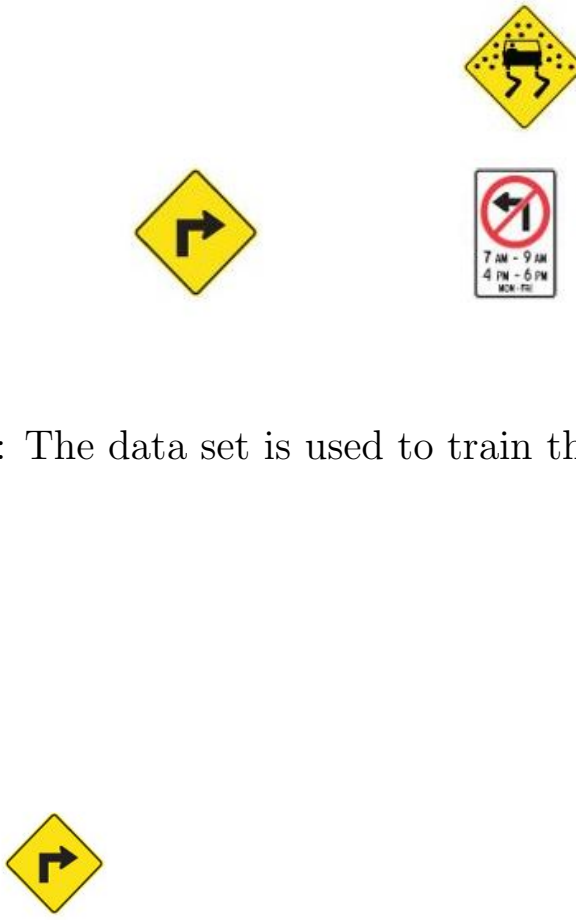

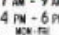
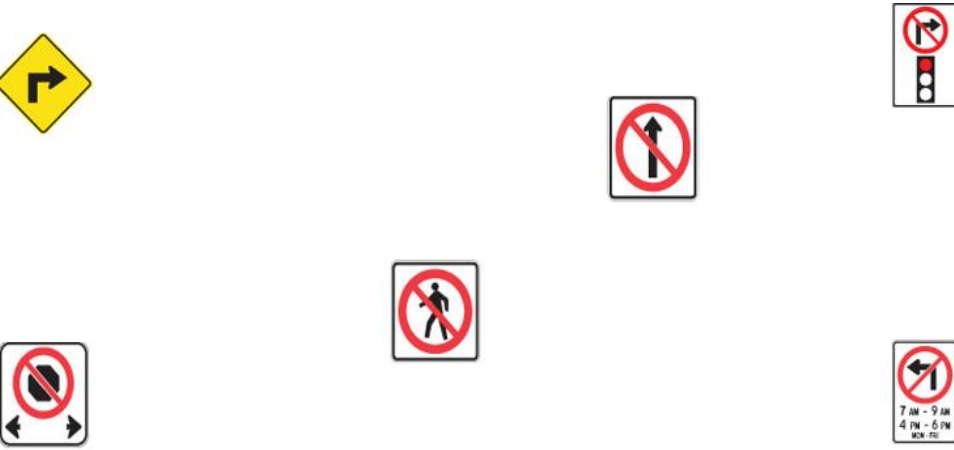

(4)

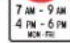
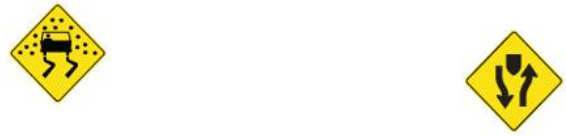

Figure 3.35: The data set is used to train the network for real life applications. 


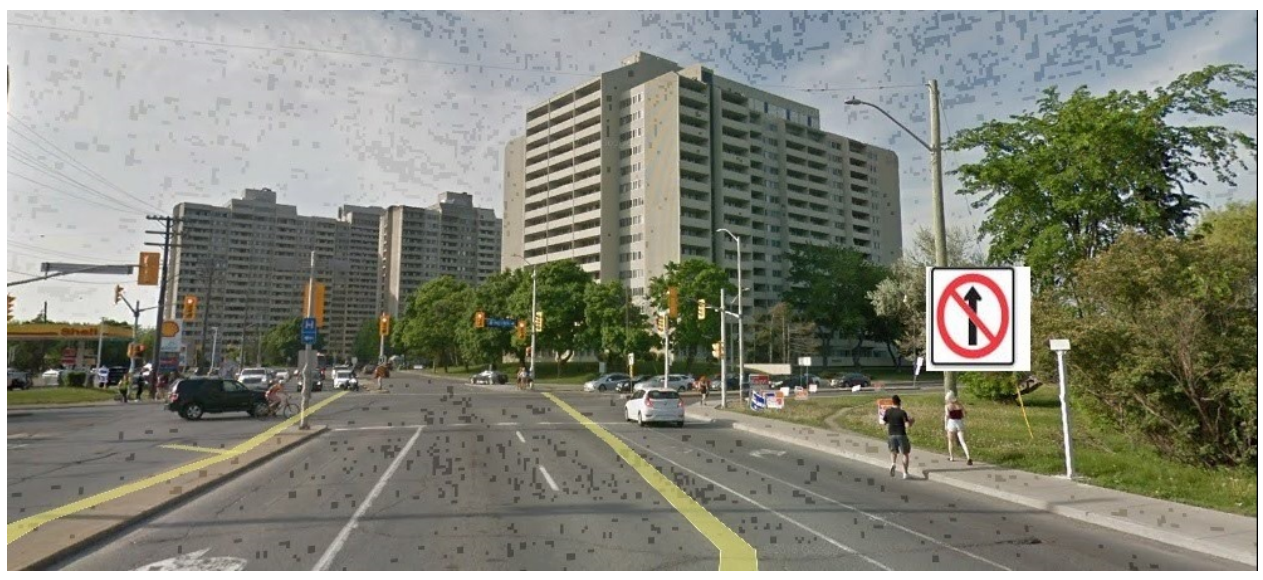

Figure 3.36: A real life picture with a sign to be recognized by the neural network.

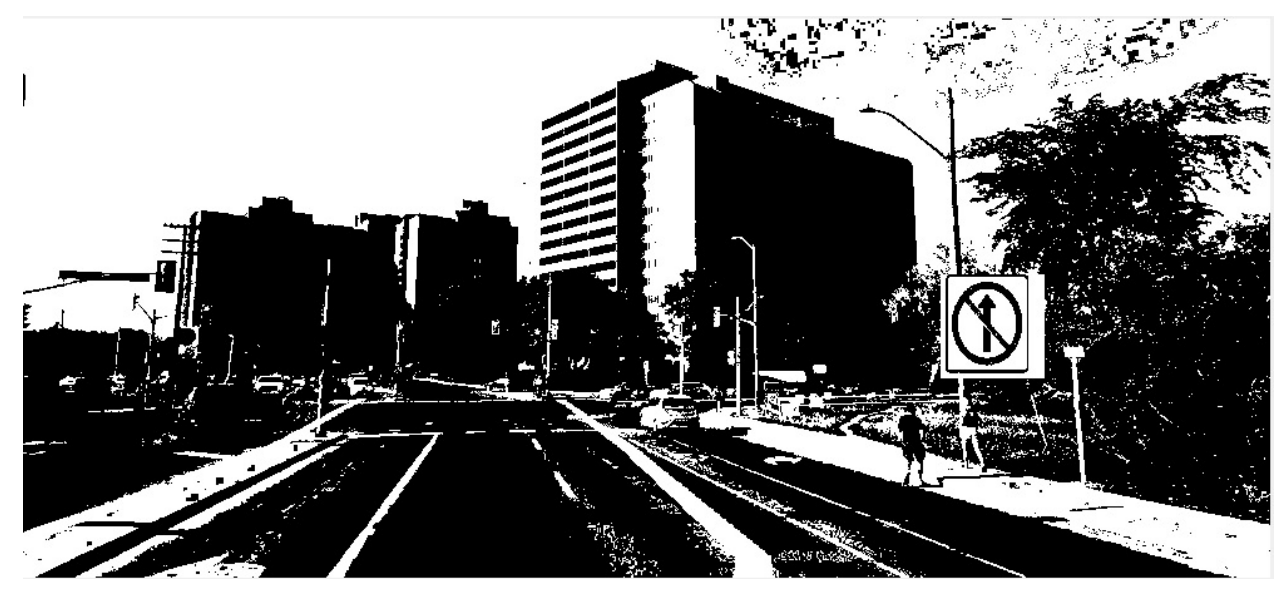

Figure 3.37: A real life picture with a sign to be recognized by the neural network. 


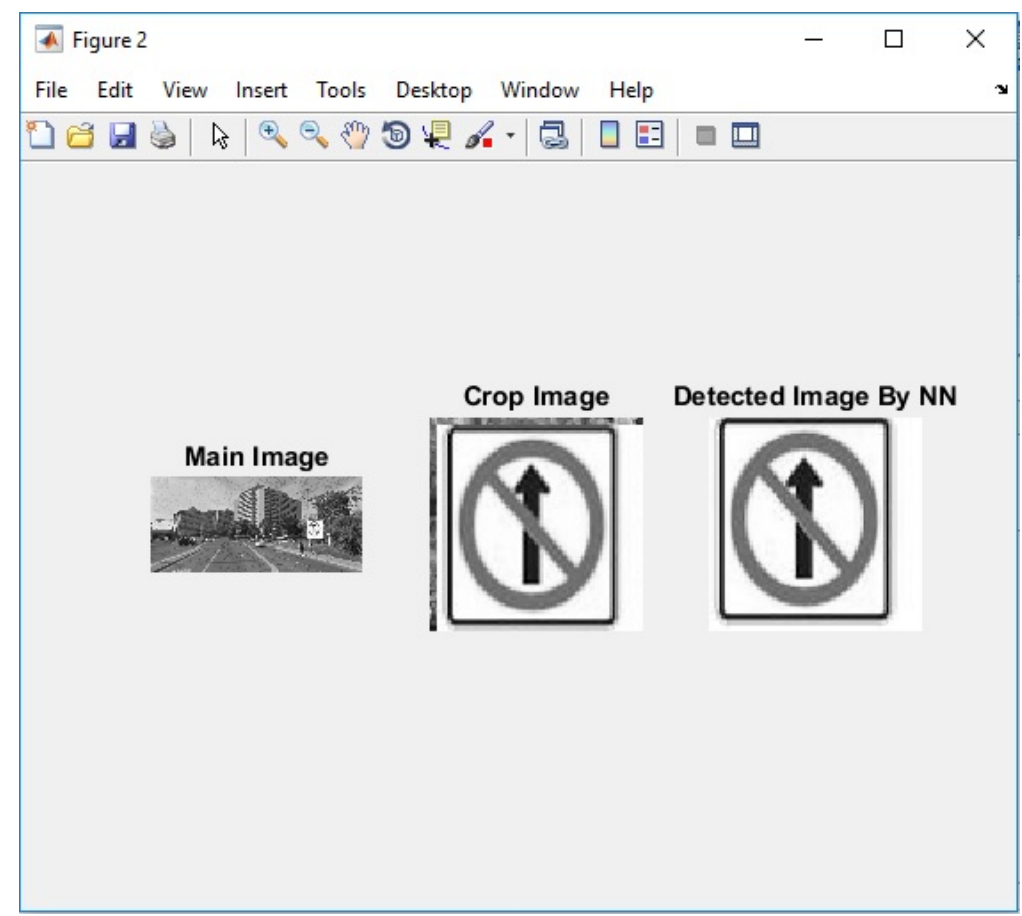

Figure 3.38: The traffic sign is recognized correctly.

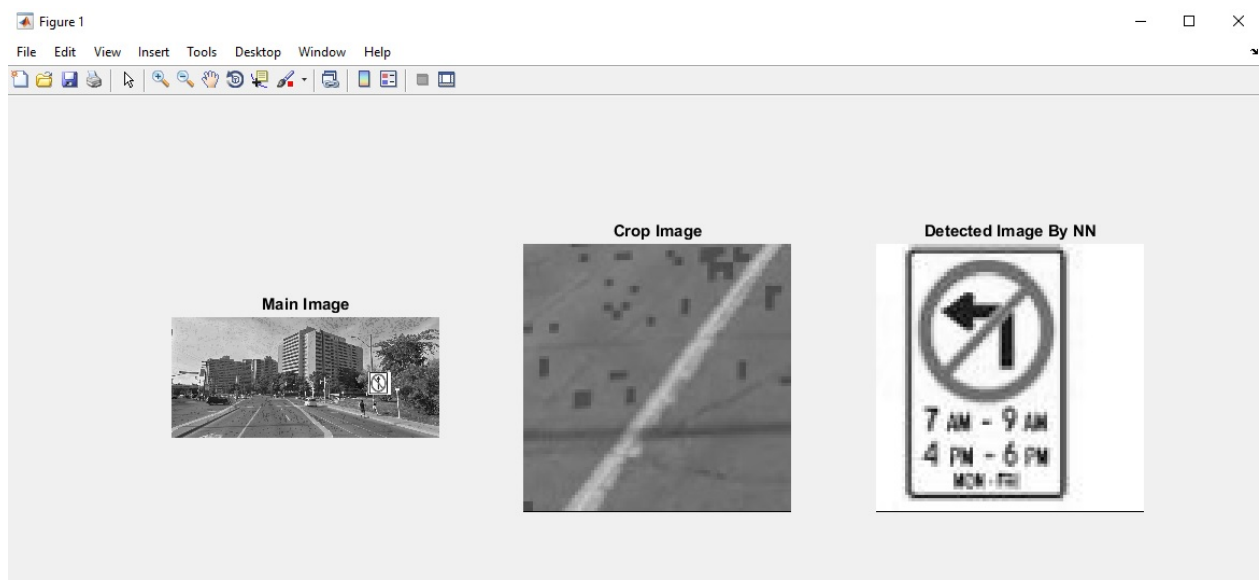

Figure 3.39: Traffic sign recognition incorrectly. 


\section{Chapter 4}

\section{Sign Detection Using LiDAR Recorded Data}

\subsection{Introduction}

One way to measure the distance to a target is to illuminate the target and measure the reflected light with a light sensor. LiDAR that stands for Light Detection And Ranging uses a laser pulse for illuminating the target and measures the return time of the reflected light using the speed of light. LiDAR could be used with other devices such as a camera to increase the accuracy of the ranging [62]. It could also be used to construct accurate $3 \mathrm{D}$ information in $\mathrm{x}, \mathrm{y}$, and $\mathrm{z}$-axis 63 despite the use of sequence of light pulses. Fig 4.1 phows visualization of recorded 3D information by LiDAR.

The working principle of LiDAR and Radar technology is similar. The main difference between the two is that they use different signals to detect objects. LiDAR's transmitter sends laser pulses to the surrounding objects, and LiDAR's receiver process the reflected light particles. In some LiDAR systems, the frequency is about 


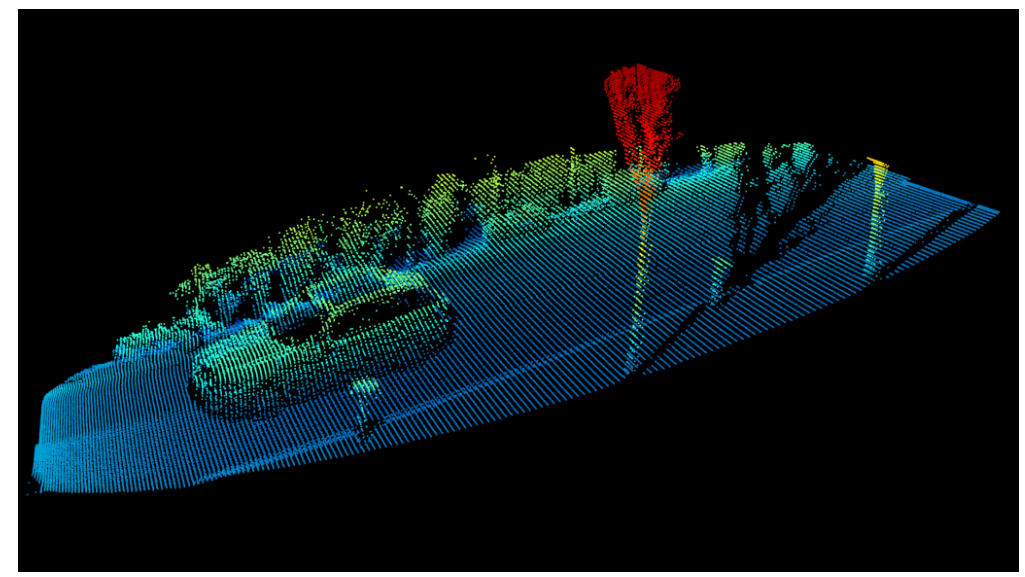

Figure 4.1: A typical visualization of LiDAR data.

64

150,000 pulse per second. The time interval between the time of sending the pulse and receiving the reflected pulse is measured by an electronic device on-board.

LiDAR is one of the most accurate technology for ranging and producing a map. This is because of using high technology of light, pulsed laser, and DSP techniques. While these high tech components are expensive, the price is expected to reduce with mass production as LiDAR becomes more and more popular in many applications.

\subsection{LiDAR for Mobility Applications}

Utilizing LiDAR for automobile applications is a relatively new trend. When it was first invented, LiDAR was most notably utilized for measuring the distance between the earth and the moon. Also it has been used to measure weather systems measurements. Today, LiDAR can be employed for any application that requires distance information of an object.

LiDAR is used for many applications as Agriculture, Archaeology, Autonomous Vehicles, Biology, Conservation, Geology, Soil science, Atmosphere, Law Enforcement, Military, Mining, Physics, Astronomy, Rock mechanics, Robotics, Spaceflight, 
Surveying, Wind Farm Optimization, Solar Photovoltaic Deployment Optimization, Video Games and the last but not the least Transportation [65].

We apply LiDAR for autonomous vehicle, which is part of smart transportation [66] [67]. Fig, 4.2 shows a LiDAR visualization which could be used for recognizing street signs in an autonomous vehicle application.

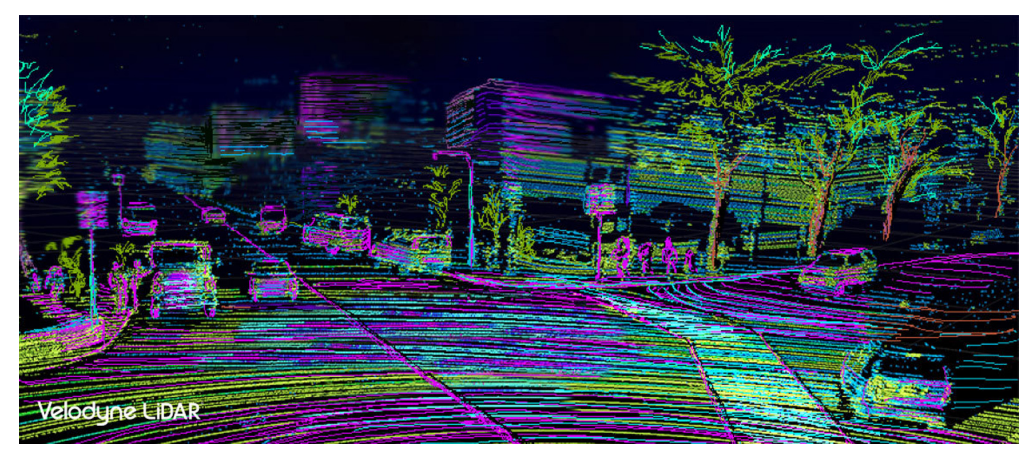

Figure 4.2: A typical visualization of LiDAR for smart transportation 68 .

LiDAR might be considered as one of the technologies that facilitate the development of smart transportation [69] [70]. LiDAR produces a large dataset that can be managed, visualized, analyzed, and shared as part of autonomous vehicle operation. However, the dataset quality drops with poor weather condition, e.g., snow, fog, rain, and dust particles. LiDAR needs to be complemented with recognition capability as it cannot detect color or contrast. As a result, in this thesis, we apply the recognition capabilities of the neural network on camera pictures to complement LiDAR's application for autonomous vehicles.

Many self-driving car manufacturers, e.g., Google, couple LiDAR with secondary sensors such as cameras or ultrasonic sensors. Fig 4.3 shows a typical use case of combined LiDAR and camera in transportation.

Velodyne LiDAR, which is a Silicon Valley-based LiDAR spun off from Velodyne 


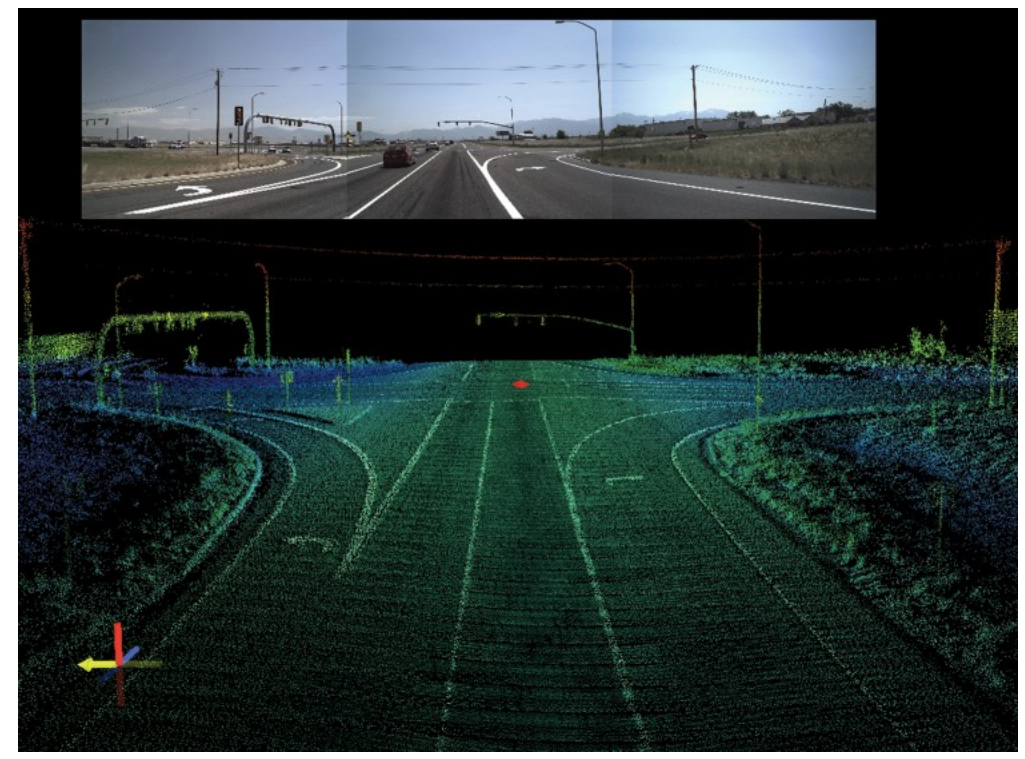

Figure 4.3: A typical combination of LiDAR and camera

[71]

Acoustics, owns LiDAR technology. The company has been working with 25 selfdriving car programs [72]. Fig 4.4 shows a Velodyne sensor is.

\subsection{LiDAR in Carleton Connected Autonomous Vehicle (CANAL)}

Autonomous vehicles typically use LiDAR for obstacle detection to navigate safely on the roads or streets. Point cloud outputs from the LiDAR sensor provide the necessary data that determines obstacles. Many companies and scientific institution such as Singapore's Singapore-MIT Alliance for Research and Technology (SMART) are actively developing technologies for autonomous LiDAR vehicles [72] Fig.4.5 shows a typical autonomous vehicle equipped with LiDAR technology.

The data collected by LiDAR can help in either making the vehicle fully smart 


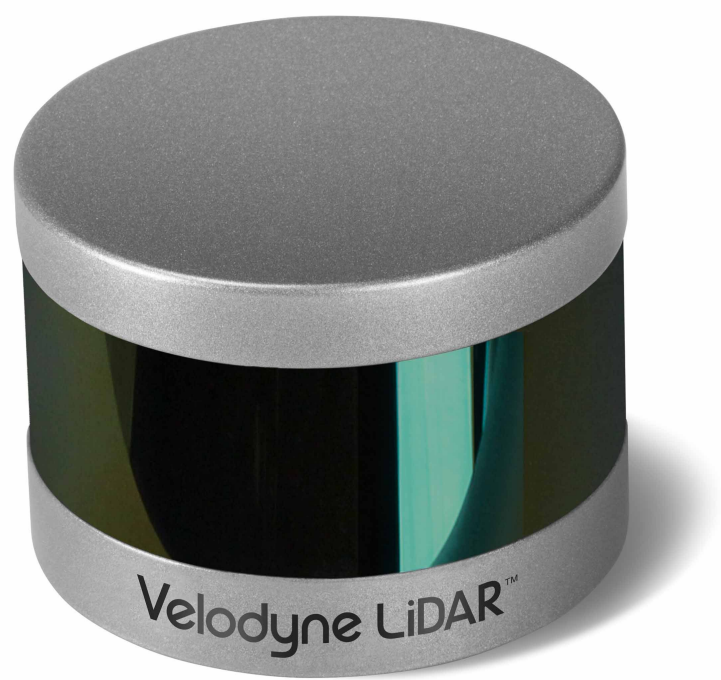

Figure 4.4: Velodyne sensor[72].

and autonomous or establishing a driver assistant system. The data is analyzed to avoid obstacles, or to assist both the adaptive cruise control system, and the anti-lock braking system. The aids provided by driver assistant system enhance safety in semismart vehicles as it offers driver with advance knowledge about what's happening around vehicle.

The major advantage of using LiDAR is that the spatial structure is obtained, and this data can be fused with other sensors such camera, etc., to get a better picture of the vehicle environment in terms of static and dynamic properties of the objects present in the environment [69]. Conversely, a significant issue with LiDAR is the difficulty in reconstructing point cloud data in poor weather conditions. In heavy rain, for example, the light pulses emitted from the LiDAR system are partially reflected off of rain droplets, adding noise called "echoes" to the data[65]. In the 


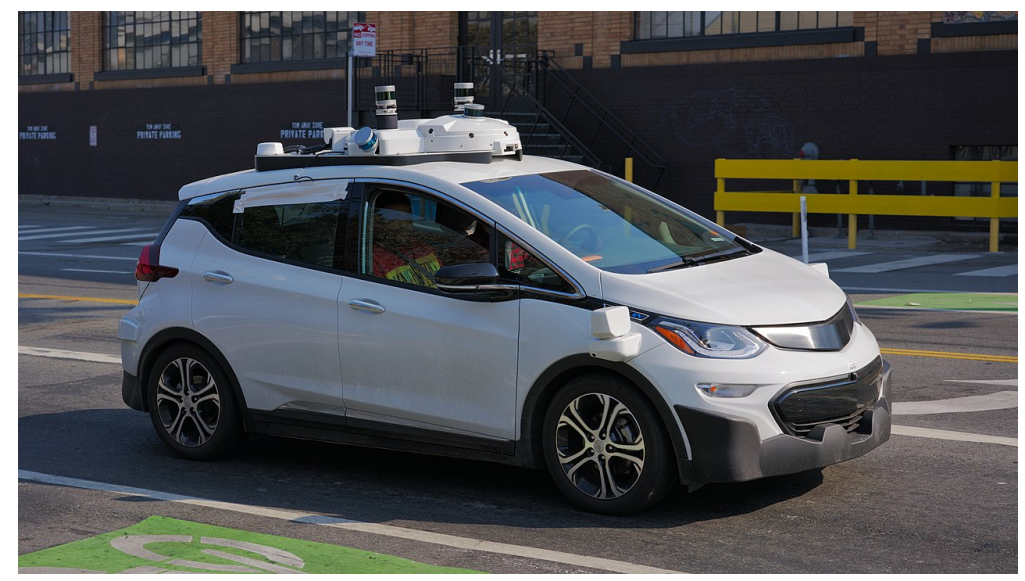

Figure 4.5: An autonomous vehicle equipped with LiDAR [72]

previous chapter, we introduced a neural network-based structure that can overcome problems with noise and weather conditions on picture data.

Velodyne LiDAR's technology can both be applied to autonomous vehicles and advanced driver assistance systems. We equipped Toyota Rav4 with Velodyne LiDAR system and record the collected data for various rides around The University campus.

The hardware and software of the CANAL were describe in Chapter 2 in detail. We analyze the LiDAR recorded to detect the signs. Different methods have been proposed for sign detection with LiDAR data[73][74]. The recent method has been employed in this thesis for sign detection based on the recorded LiDAR data in the Carleton University campus. Given the large amount of recorded data by LiDAR, we filter out the relevant data for the traffic signs. As the traffic and road signs are painted with shiny materials, the LiDAR data for the signs usually possess a minimum intensity of the points. This factor can be used for preprocessing and filtering of the data[74]. We use this approach for sign detection from recorded data. 


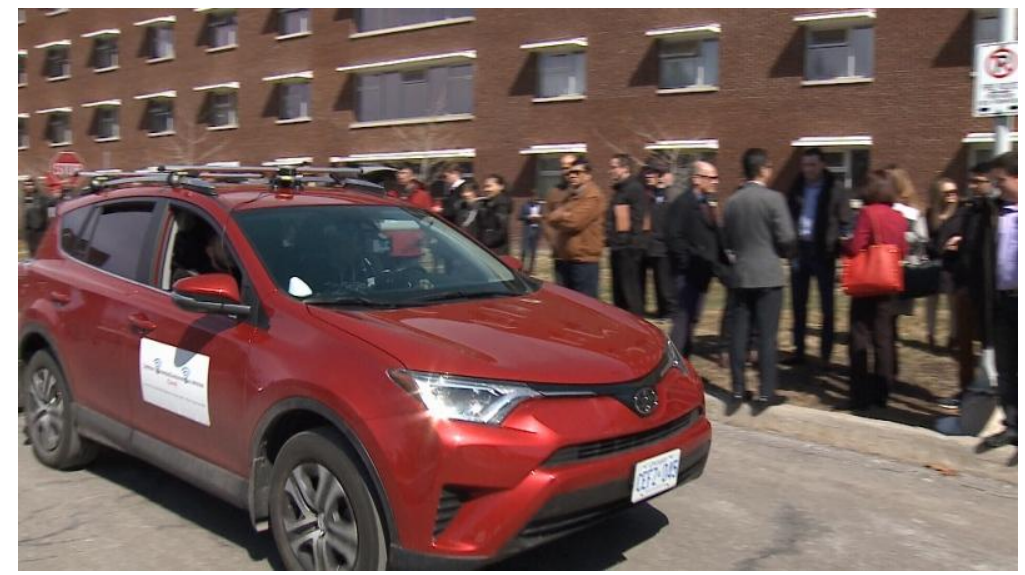

Figure 4.6: Carleton Autonomous Vehicle CANAL

\subsection{Practical and Simulation Results}

The testbed is the same as introduced in Chapter 2. Fig 4.6 shows the Toyota Rav4 (CANAL) equipped with LiDAR.

To collect required data for sign detection, the Toyota rav4 equipped with LiDAR was driven 25 times around the campus. Each drive data is recorded in one .pcap file. Figures 4.7 to 4.11 show visualization of some of the collected data.

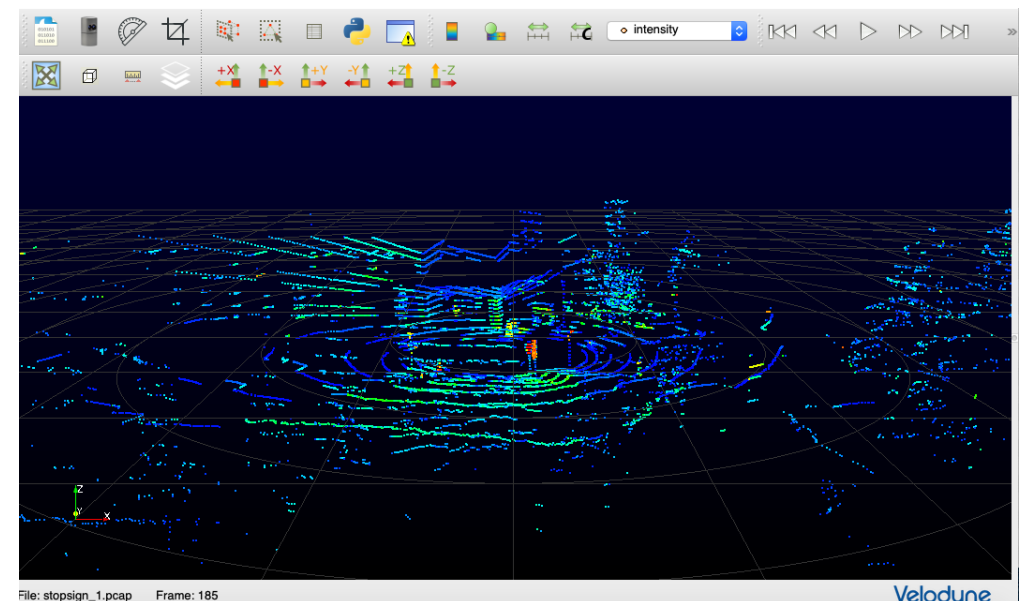

Figure 4.7: A frame of captured data recorded in Carleton University campus,Ride\#1 


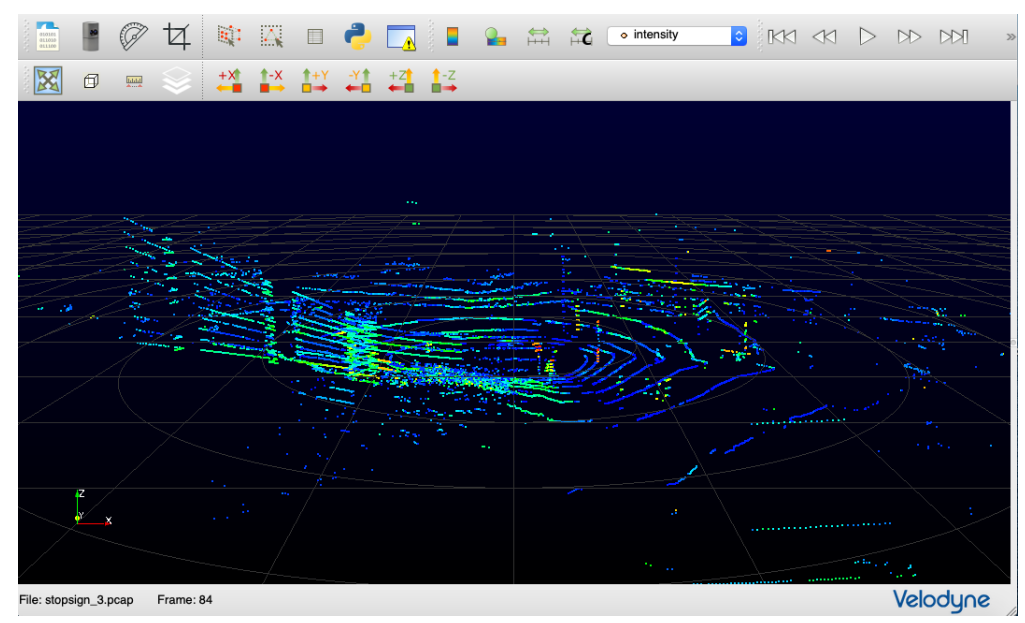

Figure 4.8: A frame of captured data recorded in Carleton University campus,Ride\#3

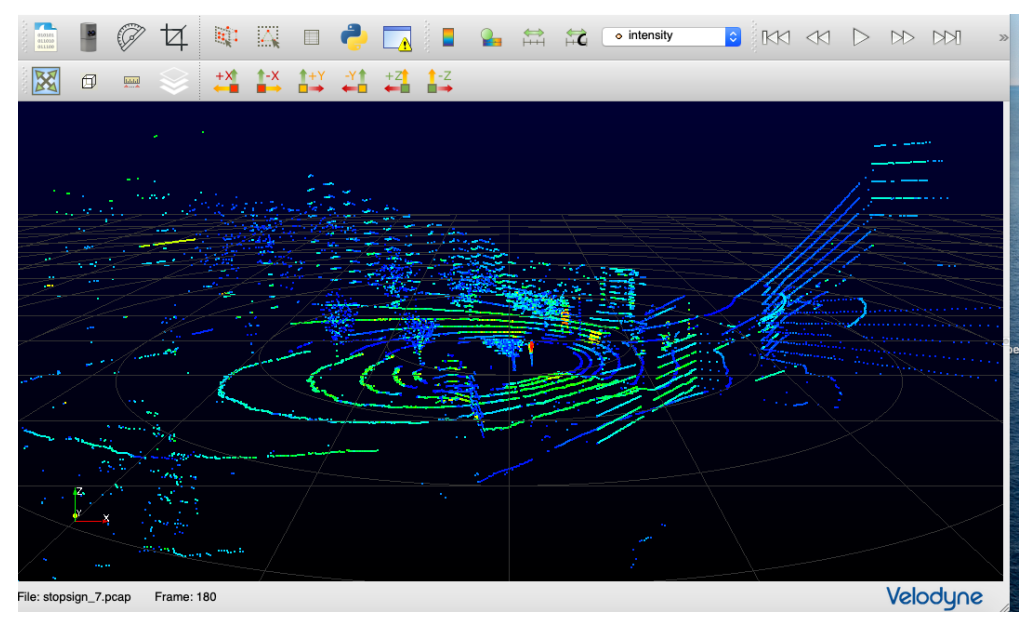

Figure 4.9: A frame of captured data recorded in Carleton University campus,Ride\#7

As seen in the figures, it is hard or even impossible to recognize which part of the figure is about a traffic and/or road sign, and more data processing is needed to assist the driver for a safe drive. At the first stage, we use a program [74], to identify the location of sign in the captured picture. A message, "sign is detected" is output to show that the first stage has been completed successfully. Figs. 4.12 - 4.14 shows the result of the program when it is applied to the captured data. As shown in the 


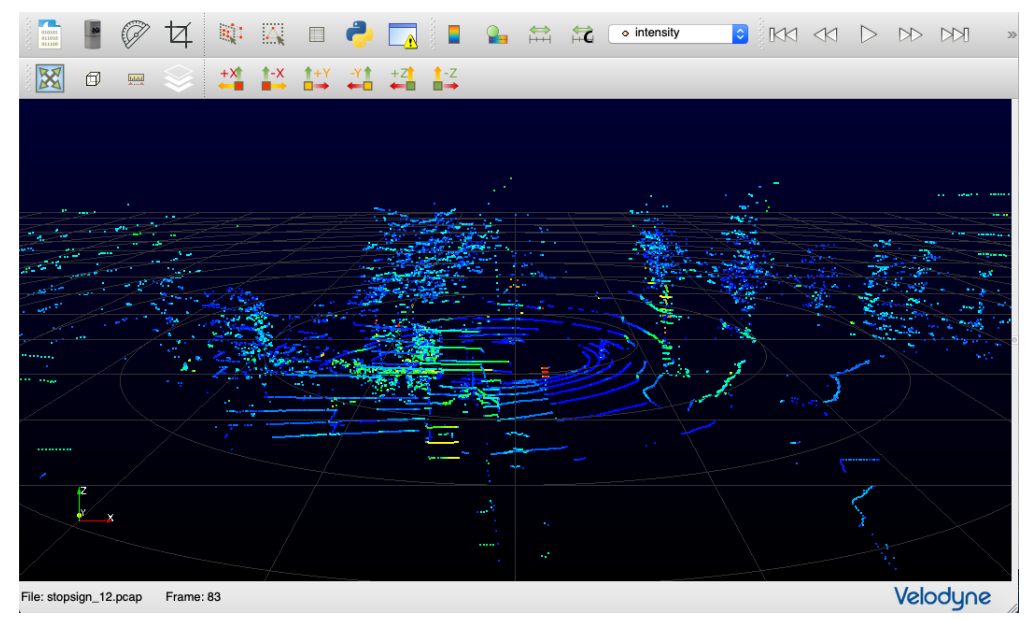

Figure 4.10: A frame of captured data recorded in Carleton University campus, Ride \#12

figures, the program has detected all the signs successfully. 


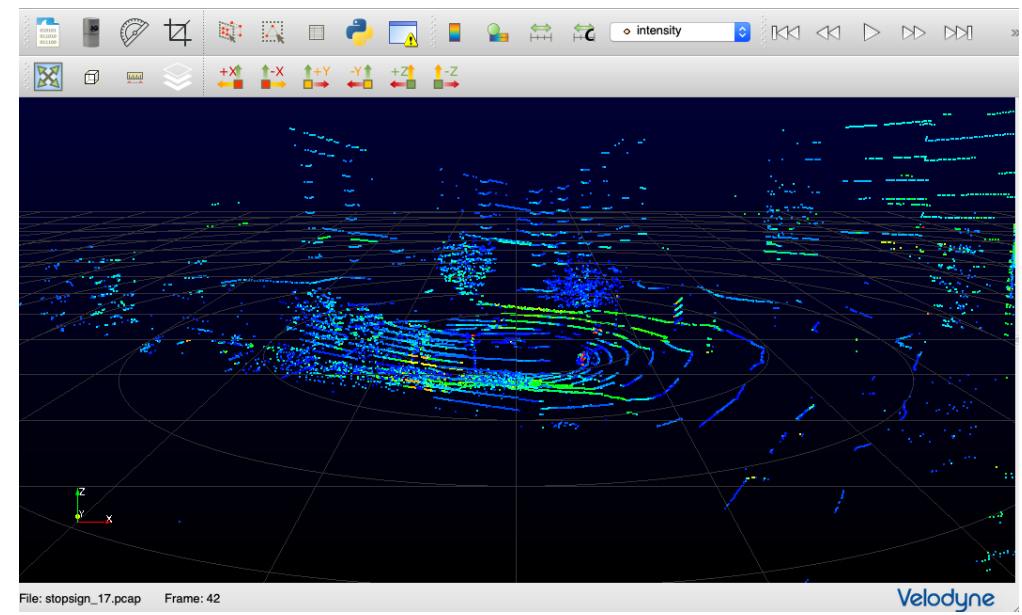

Figure 4.11: A frame of captured data recorded in Carleton University campus, Ride \#17

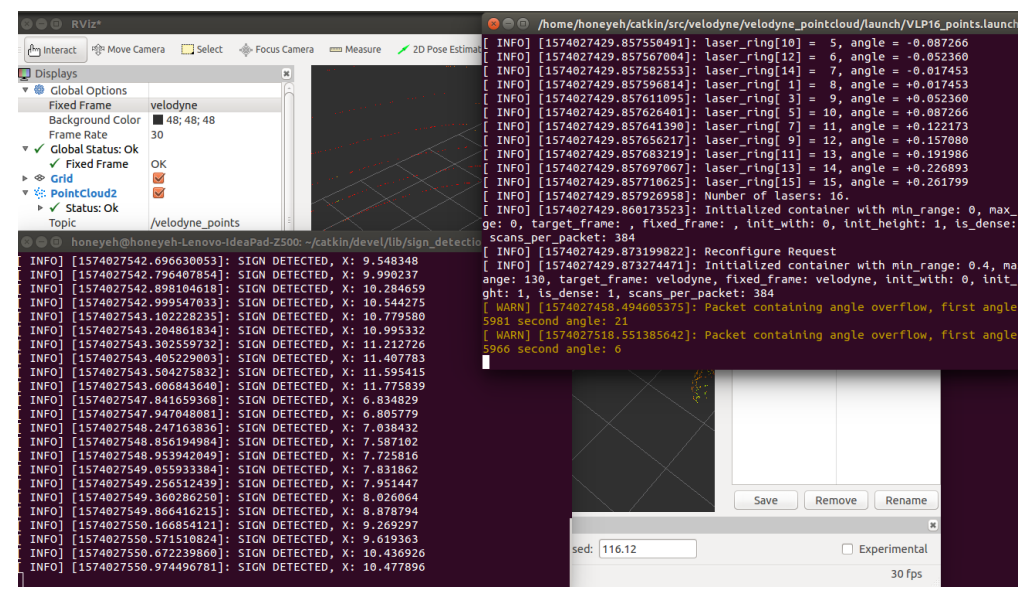

Figure 4.12: Simulation results for sign detection based on the LiDAR recorded data in Carleton Campus 


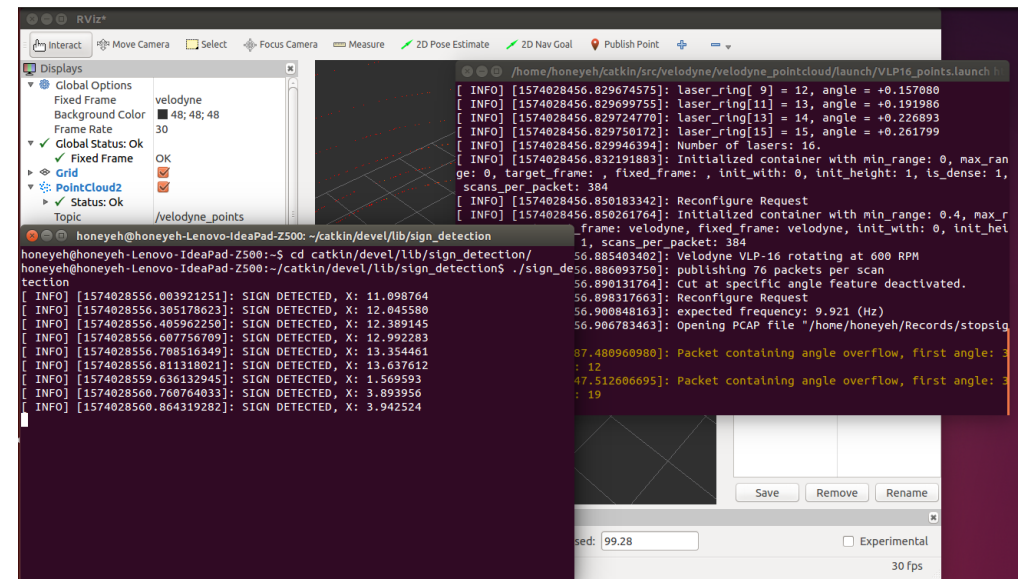

Figure 4.13: Simulation results for sign detection based on the LiDAR recorded data in Carleton Campus

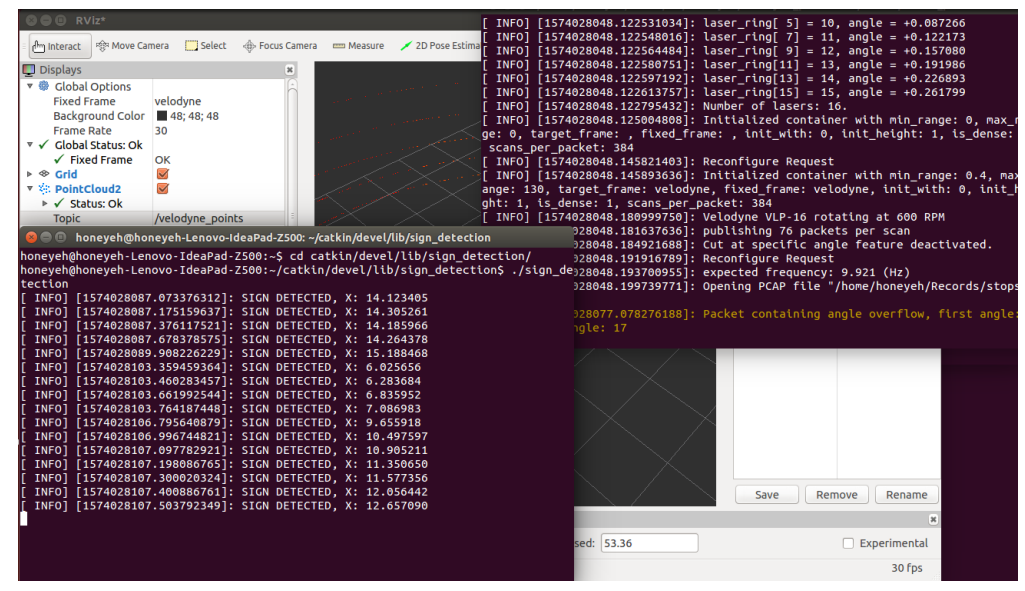

Figure 4.14: Simulation results for sign detection based on the LiDAR recorded data in Carleton Campus 


\section{Chapter 5}

\section{Conclusions and Future works}

\subsection{Concluding Remarks}

Autonomous vehicles are becoming more popular in different cities and countries. It appears that driverless vehicles play an important role in the future smart cities as part of smart transportation. An important issue of concern in deployment of driverless vehicles is detection accuracy as part of safety. In this thesis, we have taken steps toward the realization of driverless vehicles in three stages.

In stage 1, we designed a hardware and software platform that can be easily mounted and connected to any car, e.g., Toyota Rav4, as the vehicle of choice for the testbed used in the thesis. We have shown that a regular vehicle, i.e., a non-electric vehicle, may be converted to an autonomous vehicle by employing proper flexible and user-friendly hardware and software. This is an important issue because nowadays millions of regular vehicles are being used in the world, and it is economical to convert the existing vehicles to autonomous ones instead of scraping the used cars and paying for the new brand ones. A regular Toyota Rav4 car has been converted to an autonomous vehicle via an inexpensive hardware configuration. The proposed hardware 
and software structure may be used as a practical testbed for examining research issues of autonomous vehicles such as security algorithms or machine learning-based algorithms for enhancing safety by better road signs recognition in harsh weather conditions.

In stage 2, our objective is to prepare the driverless car for all seasons of Canada's weather. We proposed and designed a neural network capable of recognizing the signs even with the presence of $90 \%$ noise. The high level of added noise to traffic signs, in reality, emulates the harsh weather condition, when it is tough to recognize the traffic and road signs. We propose a neural network architecture, GRNN, for pattern recognition. The proposed neural network performs very well with $100 \%$ correct recognition for all of the available signs, each under different amounts of induced noise. In addition, the program is modified to recognize a rotated sign with noise. The simulation results show that the GRNN recognizes the signs very well when it is trained off-line based on the proper data set. To show real-life application, we also modified the network and trained it in such a way that it can recognize the sign in a street picture. The trained network can recognize the picture in some cases. More study is needed to train a better network to make sure that the network performs all the time correctly.

In stage 3 , toward a more practical system for real-life applications, we recorded the data of several rides in Carleton University campus using a LiDAR system. We could also detect the stop sign in the recorded LiDAR data, as well.

\subsection{Future Works}

Like any other research work, this work also recommends the following fields and issues to be followed in the future: 
1. To use QNX along with the existing techniques in the car for further autonomy and smartness

2. To embed the pattern recognizer into the hardware and software design of the Toyota Rav4

3. To increase the level of autonomy even more by providing more machine learning algorithms for recognizing moving objects like human-being for stopping the car properly

4. It is clear that autonomous vehicles need to detect objects such as vehicles, pedestrians, traffic signs, and so on in real-time. In order to recognize and stop the car upon detection of the stop sign practically, we have tried fast encoders for object detection by using Pointpillars. Similar to what we introduced in chapter 3 and 4, this program has to go through a training process. In order to train the system, we have used the KITTI dataset that contains 7481 samples for training and 7518 samples for testing the trained network. A Python package, named, Anaconda is required to be installed. The following steps should be followed for the training process.

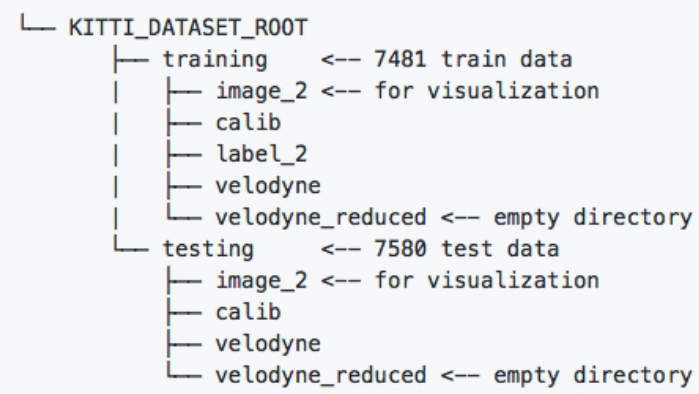

Figure 5.1: Dataset Organization [75].

Different networks need to be trained for cars and pedestrians and cyclists. It 
takes too much time to run the program while we need an online reaction. The evaluation part is then checked. The achieved results are shown in the following figure.

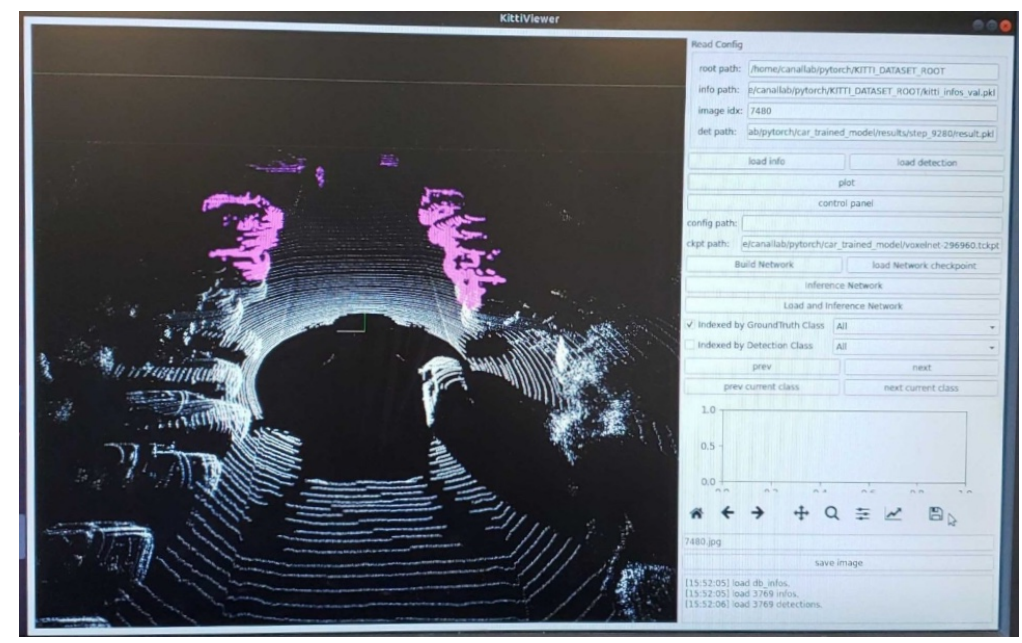

Figure 5.2: Qualitative analysis [75].

While achieving the initial results and procedure, the finishing job is needed in order to complete the goals towards a smarter autonomous vehicle. We propose this part as a future work for interested students. The other steps are almost straight forward and can be found in https://github.com/nutonomy/second.pytorch .

More LiDAR oriented research may be considered as the very promising future work of this thesis as well.

5. Data fusion for using both camera and LiDAR data to better recognize the signs may be considered as another future work. 


\section{Appendix A}

\section{Appendix}

\section{Automatic Test}

$1 \% \%$ This program which is called as Automatic-Test is used to automatically generate the data set from 58 pictures ( CANADA Trafic and Road Signs), apply them to the neural network and get the result. If there is any fault in noisy signs recognition, the program will indicate it.

2

3 clc; clear; close all \%In order to prevent mistake coming from the existing data in the work space or due to the running programs, these commands are written.

4

${ }_{5}[$ Input, Output, Image_Data_gray $]=$ Training_Data $\quad ; \quad \%$ Training data is the name of another program which is run to genertae data set for the neural netwrok.

6 
$7 \%$ The neural network which has been used for noisy trafic sign recognition is the New Genralized Regression Neural Network. Other types of neural networks like Backpropagation are not as good as NEWGRNN.

9 spread $=0.950 ; \%$ Spread is a parameter that indicates the level of accuracy which is needed. The bigger the better accuracy of the recognitin would be. It is achieved by try and error.

10

11 net $=$ newgrnn (Input, Output, spread ); \%\%The command is newgrnn that comes whith 3 arguments, namley, input which is the input to the neural network, output which is the output and spread that defines the level of desired accuracy.

12 view (net) \% The neural network which has been created by the above command is shown by view command. We can see the picture after running the program.

13 Newgrnn_Net $=$ net; \%Here the result is put in Newgrnn_Net for future usage.

14

15 correct_case $=0 ; \%$ In order to show the accuracy of the methodology we count the number of cases which has been recognized correctly.

16

${ }_{17} \%$ In the following lines, the program asks for the 
percentage amount of the noise which is needed. It can be set up to $100 \%$ !!

18

$\operatorname{disp}([$,

, ])

19 Noise_Percentage $=$ input ('How much (percentage) do you like the image to be noisy $\left.(1-100) ? \Rightarrow{ }^{\prime}\right)$;

20 $\operatorname{disp}([$,

$* * * * * * * * * * * * * * * * * * * * * * * * * * * * * * * * * * * * * * * * * * * * * * * * * * * * * * * * * * * * * * * *$

, ])

21

22

23

24 for $\mathrm{kk}=1: 1: 58$

${ }_{25}$ Input_Picture $=$ Image_Data_gray $\{k k\} ; \%$ Althogh the trafic signs are color, we need to consider them as uni-color otherwise we can not save the data in a matrix.

26 Noisy_Picture=Image_Noisy (Input_Picture, Noise_Percentage ); \% This command add some noises to the pictures. Image_Noisy is the name of the subroutine which is used to add noise 
to the picture as much as Noise_Percentage which has been predefined.

27 Net_Input_Pic $=$ double $($ reshape $($ Noisy_Picture $, 86 * 83,1)) ;$ \%ow we need to reshape the structure of the data. We put the picture data whose size is $86 * 83$ pixeles in a vector. double comand makes sure that the output is integer. $y_{-} b=$ Newgrnn_Net(Net_Input_Pic); \% In this line we input the formatted noisy picture to the network and get $y_{-} b$ as the output of the trained network.

29

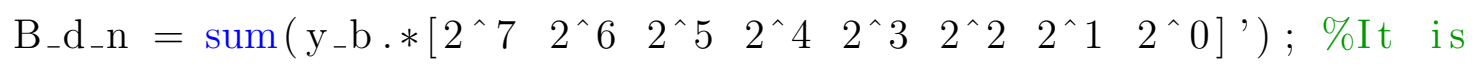
worth noting that we have 8 neurons in the output layer of the neural network, these 8 output should indicate the number of the correct picture while the noisy picture is inputto the network.

B_d_n $=$ round $\left(B_{-} d_{-} n\right) ; \% B_{-} d_{-} n$ is now the number of the correct sign which has been recognized by the network. We just round it since it has to be an integer.

31

$\%$ In the following lines we are counting the number of correct recognition .

33 if $\mathrm{kk}=\mathrm{B}_{-} \mathrm{d} \_\mathrm{n}$ 
36

37

38

39

41

42

43

44

45

47

48

49

50

els e

disp (['Loop Counter: ', num2str(kk),' Trafic sign

recognition is not ok. '])

disp (['Correct Value: ', num2str(dec2bin $(k k, 8)$ ), ' $\quad$ Predicted

Value: ', num2str(B_d_n ), , num2str (dec2bin (B_d_n

,8) ) ])

B_d_n $=59 ; \%$ We consider a sign for the time that

recognition is not done correctly. This is the 59 th sign that says wrong recognition!

end

Net_Detect_b = Image_Data_gray $\left\{B_{-}\right.$d_n_n $_{-}$;

$\%$ In this part we show the result of processing as the output for all pictures. Three pictures in a ROW are shown simultanously. The first picture is the main or noise free picture, the second one is the noisy picture and the thirs one is the picture which has been recognized by the network.

46 figure (kk)

subplot $(1,3,1)$; \%The frist picture at the left.

imshow (Input_Picture);

title ('Main Picture')

subplot $(1,3,2)$; \%The second picture in the middle. 
$51 \quad$ imshow (Noisy_Picture);

${ }_{52}$ title ('Noisy Input Picture To NN')

53 subplot $(1,3,3)$; \%The third picture at right.

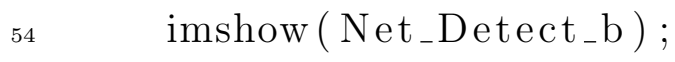

${ }_{55}$ title ('Detected Picture by NN')

56

57 end

${ }_{58} \%$ After finishing the whole process we now show the result of the performance. How many pictures out of 58 has been recognized correctly.

$59 \operatorname{disp}([$,

$* * * * * * * * * * * * * * * * * * * * * * * * * * * * * * * * * * * * * * * * * * * * * * * * * * * * * * * * * * * * * * *$

, ])

60

disp (['Number of Correctly recognized pictures: ', num2str( correct_case), ' out of all 58 pictures' $])$

$61 \operatorname{disp}([$,

$* * * * * * * * * * * * * * * * * * * * * * * * * * * * * * * * * * * * * * * * * * * * * * * * * * * * * * * * * * * * * * * *$

, ]) 


\section{Manual Test}

$1 \%$ Explanation of this program is very similar to

Automatic_Test program. Please find more explanation over

there. This program is basically written for the time we

want to test the program for some specific signs with the

desired amount of noise percentage for each.

$2 \%$ Explanation of the following lines are given in

Automatic_Test program .

3 clc; clear; close all

${ }_{4}[$ Input, Output, Image_Data_gray $]=$ Training_Data ;

${ }_{5}$ spread $=0.950 ;$

${ }_{6}$ net $=$ newgrnn (Input, Output, spread $)$;

7 view $($ net $)$

8 Newgrnn_Net $=$ net;

9

${ }_{10} \%$ In order to be able to quit the manual test, here we define User_I=0 as our desire to continue testing.

$11 \mathrm{User}_{-} \mathrm{I}=0$;

12 while User_I $=0$

$13 \mathrm{ClC}$

$14 \operatorname{disp}([$,

$* * * * * * * * * * * * * * * * * * * * * * * * * * * * * * * * * * * * * * * * * * * * * * * * * * * * * * * * * * * * * * *$

, ])

15 aa=input ('Picture Number(1:58): '); \%This line is to select the number of the sign. 
${ }_{16}$ Noise_Percent $=$ input ('How much (percentage) you want the image to be noisy $(1-100) ? \Rightarrow \quad$ ') ; \% This line is to indicate the perventage amount of the noise.

17

$\operatorname{disp}([$,

$* * *$

, ])

18

$19 \%$ This is to make sure that the number of the sign is a valid number .

20 if aa $>58$

$21 \quad \mathrm{aa}=58$

22

23

24

25

26

27

28 Noisy_Picture=Image_Noisy (Input_Picture, Noise_Percent); \%

29 Net_Input_Pic $=$ double $($ reshape $($ Noisy_Picture $, 86 * 83,1)) ; \%$ This

els eif aa $<=0$

aa $=1$

end

(1)

(1)

(1)

Input_Picture = Image_Data_gray $\{$ aa $\} ; \%$ As indicated in the other program this is to make all the signs uni-color. Image_Noisy command is to run the program with the same name and make the original picyures noisy. is to reshape them and make them ready to be sent to the neural network.

30 $\mathrm{y}_{-} \mathrm{b}=$ Newgrnn_Net (Net_Input_Pic) ; \%The output of the neural 
network .

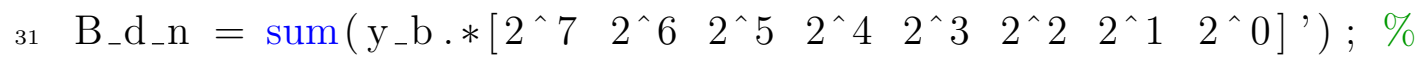

Findingthe number of sign from the 0\&1's which are coming out of the network.

32

33

34

35

36

37

38

39

if $\left(\left(B_{-} d_{-} n^{\sim}=\right.\right.$ aa $\left.)\right)$ \%This is actually the case where the sig has not been recognized correctly and we go the positio 59 which is associated to wrong picture.

$$
\text { B_d_n }=59 ;
$$

end

Net_Detect_b $=$ Image_Data_gray $\left\{B_{-} d_{-} n\right\}$;

$\%$ Here we show the result.

figure

subplot $(1,3,1)$;

imshow (Input_Picture);

title ('Main Picture')

subplot $(1,3,2)$;

imshow ( Noisy_Picture);

title ('Noisy Input Picture To NN')

subplot $(1,3,3)$;

imshow (Net_Detect_b);

title ('Detected Picture By NN') 
${ }_{51} \%$

${ }_{52} \operatorname{disp}([$,

$* * * * * * * * * * * * * * * * * * * * * * * * * * * * * * * * * * * * * * * * * * * * * * * * * * * * * * * * * * * * * * *$

, ])

${ }_{53}$ User_I $=$ input(' If you want to continue the test, Press 0 , else Press other Keys $\Longrightarrow \quad$ ') ; \%In order to start a new test we need to keep User_I $==0$

54 end 


\section{Training Data}

$1 \%$ Neural network-based methods are highly dependent to the availability of data for traingn. It is very important to have large amount of data for traingn the network in order to get accurate response. For each road or trafic sign we need to use the original sign itself and its noisy ones as well. We have made each picture noisy by adding $5 \%$ up to $95 \%$ noise by the step of $5 \%$. Therefore, for each picture we have $19=95 / 5$ noisy signs. We can use each noisy sign twice or even more for traing the network. Here we have used twice so the size is $19 * 2=38$.

2

3 function [Input, Output, Image_Data_gray]=Training_Data \%This is to set up the training data.

${ }_{4} \mathrm{j} \mathrm{j}=1 ;$

5 for $\mathrm{ii}=1: 58 \% 58$ is the number of signs.

$6 \quad$ Image_Data $\{$ i i $\}=$ imread (['Guide_Pictures $\backslash \mathrm{P}_{-}$',, num2str(ii) '.jpg']); \%Original picture

$7 \quad$ II $=$ rgb2gray (Image_Data $\{$ ii $\}) ; \%$ This is to change the color sign to a black and white sign.

$8 \quad$ I_n $=$ imresize (II, $\left.\left[\begin{array}{ll}86 & 83\end{array}\right]\right) ; \quad$ \%This is to resize all the sign to a unique size which is $86 * 83$

$9 \quad$ Image_Data_gray $\{$ i i $\}=I_{-} n$;

10 Image_Data_gray_Reshape $\{$ ii $\}=$ double(reshape( Image_Data_gray $\{\mathrm{ii}\}, 86 * 83,1))$; \%This is to reshape the 
data structure as explained in the other program. $\%$ With 5\% Disturbance

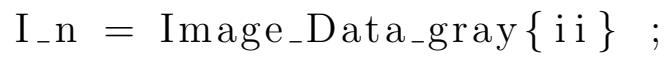

$\% \%$ Now we add $5 \%$ noise and repeat the whole process, this will be done up to $95 \%$ with the $5 \%$ step.

Noise_Percentage $=5$;

$$
I_{-} n=I m a g e \_N o i s y\left(I_{-} n,\right. \text { Noise_Percentage ) ; }
$$$$
\text { Image_Data_gray_5 }\{\text { i i }\}=I_{-} n \text {; }
$$

Image_Data_gray_Reshape_5 $\{$ i i $\}=$ double (reshape (

$$
\text { Image_Data_gray_5\{ i i }\}, 86 * 83,1)) \text {; }
$$

$\%$ This is done twice (for $5 \%$ noise) in order to get a reacher training set.

I_n $=$ Image_Data_gray $\{$ i i $\}$;

Noise_Percentage $=5$;

I_n=Image_Noisy ( I_n, Noise_Percentage );

Image_Data_gray_5_2 2 i i $\}=I_{-} n$;

Image_Data_gray_5_2 $\{$ i i $\}, 86 * 83,1))$;

$\%$ With $\% 10$ Disturbance

$I_{-} n=$ Image_Data_gray $\{$ i i $\} ;$

$\%$ Now the program is repeated for $10 \%$ noise.

Noise_Percentage $=10 ;$

I_n=Image_Noisy ( I_n, Noise_Percentage );

Image_Data_gray_10 $\left.\mathrm{ii}_{-}\right\}=\mathrm{I}_{-} \mathrm{n}$;

Image_Data_gray_Reshape_10 $\{\mathrm{i}$ i $\}=$ double (reshape ( 
Image_Data_gray_10\{ i i $\}, 86 * 83,1))$;

$\%$ With \%10_2 Disturbance

$\mathrm{I}_{-} \mathrm{n}=\operatorname{Image}_{-}$Data_gray $\{\mathrm{i} \mathrm{i}\}$;

Noise_Percentage $=10 ;$

I_n=Image_Noisy ( I_n, Noise_Percentage ) ;

Image_Data_gray_10_2 $\{$ i i $\}=I_{-} n$;

Image_Data_gray_Reshape_10_2 $\{\mathrm{i} i\}=$ double (reshape (

Image_Data_gray_10_2\{ i i $\}, 86 * 83,1))$;

$\%$ With 15\% Disturbance

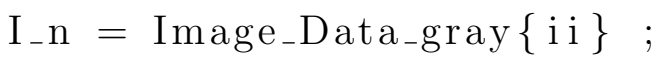

$\%$ Now the program is repeated for $15 \%$ noise.

Noise_Percentage $=15$;

I_n=Image_Noisy ( I_n, Noise_Percentage );

Image_Data_gray_15\{ $\mathrm{i}$ i $\}=\mathrm{I}_{-} \mathrm{n}$;

Image_Data_gray_Reshape_ $15\{$ i i $\}=$ double (reshape (

Image_Data_gray_15\{ i i $\}, 86 * 83,1))$;

$\%$ With 15_2 Disturbance

I_n $=$ Image_Data_gray $\{$ i i $\}$;

Noise_Percentage $=15$;

I_n=Image_Noisy (I_n, Noise_Percentage );

Image_Data_gray_15_2 $\{$ i i $\}=I_{-} n$;

Image_Data_gray_Reshape_15_2 $\{\mathrm{i} i\}=$ double (reshape (

Image_Data_gray_15_2\{ i i $\}, 86 * 83,1))$;

$\%$ With \%20 Disturbance

I_n $=$ Image_Data_gray $\{$ i i $\}$; 
$\%$ Now the program is repeated for $20 \%$ noise.

Noise_Percentage $=20 ;$

I_n=Image_Noisy ( I_n, Noise_Percentage ) ;

Image_Data_gray_20 $\left.\mathrm{i}_{-} \mathrm{i}\right\}=\mathrm{I}_{-} \mathrm{n}$;

Image_Data_gray_Reshape_20 $\{$ i i $\}=$ double (reshape (

Image_Data_gray_20 $\{$ i i $\}, 86 * 83,1))$;

$\%$ With \%20_2 Disturbance

I_n $=$ Image_Data_gray $\{$ i i $\}$;

Noise_Percent $=20$;

I_n=Image_Noisy ( I_n, Noise_Percentage ) ;

Image_Data_gray_20_2 $\{\mathrm{i}$ i $\}=I_{-} n$;

Image_Data_gray_Reshape_20_2 $\{\mathrm{i} i\}=$ double (reshape (

Image_Data_gray_20_2\{ i i $\}, 86 * 83,1))$;

$\%$ With 25\% Disturbance

I_n $=$ Image_Data_gray $\{$ i i $\}$;

$\%$ Now the program is repeated for $25 \%$ noise.

Noise_Percentage $=25$;

I_n=Image_Noisy (I_n, Noise_Percentage );

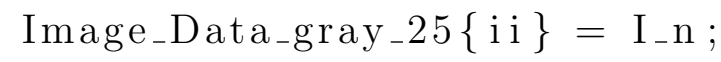

Image_Data_gray_Reshape_25\{ i i $\}=$ double (reshape (

Image_Data_gray_25\{ i i $\}, 86 * 83,1))$;

$\%$ With 25_2 Disturbance

I_n $=$ Image_Data_gray $\{$ i i $\} ;$

Noise_Percentage $=25$;

I_n=Image_Noisy ( I_n, Noise_Percentage ); 
Image_Data_gray_25_2 $2 \mathrm{ii}\}=\mathrm{I}_{-} \mathrm{n}$;

Image_Data_gray_Reshape_25_2 $\{\mathrm{i} i\}=$ double $($ reshape ( Image_Data_gray_25_2 $\{$ i i $\}, 86 * 83,1))$;

$\%$ With $\% 30$ Disturbance

I_n $=$ Image_Data_gray $\{$ i i $\}$;

$\%$ Now the program is repeated for 30\% noise.

Noise_Percentage $=30 ;$

I_n=Image_Noisy ( I_n, Noise_Percentage );

Image_Data_gray_30 $\{\mathrm{i}$ i $\}=\mathrm{I}_{-} \mathrm{n}$;

Image_Data_gray_Reshape_30 $\{$ i i $\}=$ double (reshape (

Image_Data_gray_30 $\{$ i i $\}, 86 * 83,1))$;

$\%$ With \%30_2 Disturbance

I_n $=$ Image_Data_gray $\{$ i i $\}$;

Noise_Percentage $=30 ;$

I_n=Image_Noisy ( I_n, Noise_Percentage );

Image_Data_gray_30_2 $\{\mathrm{i}$ i $\}=\mathrm{I}_{-} \mathrm{n}$;

Image_Data_gray_Reshape_30_2 $\{$ i i $\}=$ double (reshape (

Image_Data_gray_30_2 $\{$ i i $\}, 86 * 83,1))$;

$\%$ With 35\% Disturbance

I_n $=$ Image_Data_gray $\{$ i i $\}$;

$\% \%$ Now the program is repeated for $35 \%$ noise.

Noise_Percentage $=35$;

I_n=Image_Noisy (I_n, Noise_Percentage );

Image_Data_gray_35\{i i $\}=I_{-} n$;

Image_Data_gray_Reshape_35\{ i i $\}=$ double(reshape ( 
Image_Data_gray_35\{ i i $\}, 86 * 83,1))$;

$\%$ With 35_2 Disturbance

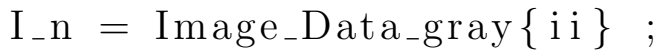

Noise_Percentage $=35$;

I_n=Image_Noisy ( I_n, Noise_Percentage ) ;

Image_Data_gray_35_2 $\{$ i i $\}=I_{-} n$;

Image_Data_gray_Reshape_35_2 $\{\mathrm{i} i\}=$ double (reshape (

Image_Data_gray_35_2\{ i i $\}, 86 * 83,1))$;

$\%$ With 40\% Disturbance

$\mathrm{I}_{-} \mathrm{n}=$ Image_Data_gray $\{\mathrm{i} \mathrm{i}\}_{\text {; }}$;

$\% \%$ Now the program is repeated for $40 \%$ noise.

Noise_Percentage $=40 ;$

I_n=Image_Noisy ( I_n, Noise_Percentage );

Image_Data_gray_40 $\{$ i i $\}=I_{-} n$;

Image_Data_gray_Reshape_40 $\{\mathrm{i} i\}=$ double $($ reshape (

Image_Data_gray_40 $\{$ i i $\}, 86 * 83,1))$;

$\%$ With \%40_2 Disturbance

I_n $=$ Image_Data_gray $\{$ i i $\}$;

Noise_Percentage $=40 ;$

I_n=Image_Noisy (I_n, Noise_Percentage );

Image_Data_gray_40_2 $\{$ i i $\}=I_{-} n$;

Image_Data_gray_Reshape_40_2 $\{\mathrm{i} i\}=$ double (reshape (

Image_Data_gray_40_2 $\{$ i i $\}, 86 * 83,1))$;

$\%$ With $45 \%$ Disturbance

I_n $=$ Image_Data_gray $\{$ i i $\}$; 
$\% \%$ Now the program is repeated for $45 \%$ noise.

Noise_Percentage $=45 ;$

I_n=Image_Noisy ( I_n , Noise_Percentage );

Image_Data_gray_45\{ $\mathrm{i}$ i $\}=\mathrm{I}_{-} \mathrm{n}$;

Image_Data_gray_Reshape_45 $\{$ i i $\}=$ double (reshape (

Image_Data_gray_45 $\{$ i i $\}, 86 * 83,1))$;

$\%$ With 45_2 Disturbance

I_n $=$ Image_Data_gray $\{$ i i $\}$;

Noise_Percentage $=45 ;$

I_n=Image_Noisy ( I_n, Noise_Percentage );

Image_Data_gray_45_2 $\{$ i i $\}=I_{-} n$;

Image_Data_gray_Reshape_45_2 $\{\mathrm{i} i\}=$ double (reshape (

Image_Data_gray_45_2\{ i i $\}, 86 * 83,1))$;

$\%$ With 50\% Disturbance

I_n $=$ Image_Data_gray $\{$ ii $\}$;

$\%$ Now the program is repeated for $50 \%$ noise.

Noise_Percentage $=50 ;$

I_n=Image_Noisy (I_n, Noise_Percentage );

Image_Data_gray_50 $\{$ i i $\}=I_{-} n$;

Image_Data_gray_Reshape_50 $\{$ i i $\}=$ double(reshape (

Image_Data_gray_50\{ i i $\}, 86 * 83,1))$;

$\%$ With \%50_2 Disturbance

I_n $=$ Image_Data_gray $\{\mathrm{i} i\}$;

Noise_Percentage $=50 ;$

I_n=Image_Noisy ( I_n, Noise_Percentage ) ; 
Image_Data_gray_50_2 $\{\mathrm{ii}\}=\mathrm{I}_{-} \mathrm{n}$;

Image_Data_gray_Reshape_50_2 $\{\mathrm{i} i\}=$ double (reshape ( Image_Data_gray_50_2 $\{$ i i $\}, 86 * 83,1))$;

$\%$ With 55\% Disturbance

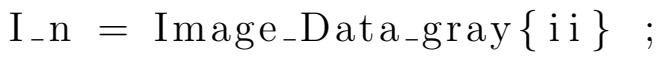

$\% \%$ Now the program is repeated for 55\% noise.

Noise_Percentage $=55$;

I_n=Image_Noisy ( I_n, Noise_Percentage );

Image_Data_gray_5 5 i i $\}=\mathrm{I}_{-} \mathrm{n}$;

Image_Data_gray_Reshape_5 $5\{$ i i $\}=$ double(reshape (

Image_Data_gray_5 $5\{$ i i $\}, 86 * 83,1))$;

$\%$ With 55_2 Disturbance

$I_{-} n=$ Image_Data_gray $\{$ i i $\}$;

Noise_Percentage $=55$

I_n=Image_Noisy (I_n, Noise_Percentage );

Image_Data_gray_55_2 $\{$ i i $\}=I_{-} n$;

Image_Data_gray_Reshape_55_2 $\{\mathrm{i} i\}=$ double (reshape (

Image_Data_gray_55_2 $\{$ i i $\}, 86 * 83,1))$;

$\%$ With 60\% Disturbance

I_n $=$ Image_Data_gray $\{$ i i $\}$;

$\% \%$ Now the program is repeated for $60 \%$ noise.

Noise_Percentage $=60 ;$

I_n=Image_Noisy ( I_n, Noise_Percentage );

Image_Data_gray_60 $\{\mathrm{i}$ i $\}=I_{-} n$;

Image_Data_gray_Reshape_60 $\{$ i i $\}=$ double (reshape ( 
Image_Data_gray_60\{ i i $\}, 86 * 83,1))$;

$\%$ With \%60_2 Disturbance

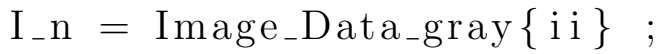

Noise_Percentage $=60 ;$

I_n=Image_Noisy ( I_n, Noise_Percentage ) ;

Image_Data_gray_60_2 $\{$ i i $\}=I_{-} n$;

Image_Data_gray_Reshape_60_2 $\{\mathrm{i} i\}=$ double (reshape (

Image_Data_gray_60_2\{ i i $\}, 86 * 83,1))$;

$\%$ With 65\% Disturbance

I_n $=$ Image_Data_gray $\{\mathrm{i} i\}$;

$\%$ Now the program is repeated for $65 \%$ noise.

Noise_Percentage $=65$;

I_n=Image_Noisy ( I_n, Noise_Percentage );

Image_Data_gray_65 $\left.\mathrm{ii}_{-}\right\}=\mathrm{I}_{-} \mathrm{n}$;

Image_Data_gray_Reshape_65 $\{$ i i $\}=$ double (reshape (

Image_Data_gray_65\{ i i $\}, 86 * 83,1))$;

$\%$ With 65_2 Disturbance

I_n $=$ Image_Data_gray $\{\mathrm{i} i\}$;

Noise_Percentage $=65 ;$

I_n=Image_Noisy (I_n, Noise_Percentage );

Image_Data_gray_65_2 $\{$ i i $\}=I_{-} n$;

Image_Data_gray_Reshape_65_2 $\{\mathrm{i} i\}=$ double (reshape (

Image_Data_gray_65_2 $\{$ i i $\}, 86 * 83,1))$;

$\%$ With 70\% Disturbance

I_n $=$ Image_Data_gray $\{$ i i $\}$; 
$\% \%$ Now the program is repeated for $70 \%$ noise.

Noise_Percentage $=70 ;$

I_n=Image_Noisy ( I_n , Noise_Percentage );

Image_Data_gray_70\{i i $\}=\mathrm{I}_{-} \mathrm{n}$;

Image_Data_gray_Reshape_70 $\{$ i i $\}=$ double(reshape (

Image_Data_gray_70 $\{$ i i $\}, 86 * 83,1))$;

$\%$ With $70 \_2$ Disturbance

I_n $=$ Image_Data_gray $\{$ i i $\}$;

Noise_Percentage $=70 ;$

I_n=Image_Noisy ( I_n, Noise_Percentage );

Image_Data_gray_70_2 $\{$ i i $\}=I_{-} n$;

Image_Data_gray_Reshape_70_2 $\{\mathrm{i} i\}=$ double (reshape (

Image_Data_gray_70_2 2 i i $\}, 86 * 83,1))$;

$\%$ With $75 \%$ Disturbance

I_n $=$ Image_Data_gray $\{$ i $i\}$;

$\%$ Now the program is repeated for $75 \%$ noise.

Noise_Percentage $=75$;

I_n=Image_Noisy (I_n, Noise_Percentage );

Image_Data_gray_75\{ i i $\}=I_{-} n$;

Image_Data_gray_Reshape_75 $\{$ i i $\}=$ double (reshape (

Image_Data_gray_75\{ i i $\}, 86 * 83,1))$;

$\%$ With 75_2 Disturbance

I_n $=$ Image_Data_gray $\{$ i i $\}$;

Noise_Percentage $=75$;

I_n=Image_Noisy ( I_n, Noise_Percentage ); 
Image_Data_gray_ $75_{-} 2\{\mathrm{ii}\}=\mathrm{I}_{-} \mathrm{n}$;

Image_Data_gray_Reshape_ $75_{-} 2\{\mathrm{i} i\}=$ double $($ reshape ( Image_Data_gray_ $75 \_2\{$ i i $\left.\left.\}, 86 * 83,1\right)\right)$;

$\%$ With $80 \%$ Disturbance

$I_{-} n=I_{m a g e}$ Data_gray $\{$ i i $\} ;$

$\%$ Now the program is repeated for $80 \%$ noise.

Noise_Percentage $=80 ;$

I_n=Image_Noisy ( I_n, Noise_Percentage );

Image_Data_gray_80 $\{\mathrm{i}$ i $\}=\mathrm{I}_{-} \mathrm{n}$;

Image_Data_gray_Reshape_ $80\{$ i i $\}=$ double (reshape (

Image_Data_gray_80 $\{$ i i $\}, 86 * 83,1))$;

$\%$ With $80 \_2$ Disturbance

I_n $=$ Image_Data_gray $\{$ i i $\}$;

Noise_Percentage $=80 ;$

I_n=Image_Noisy ( I_n, Noise_Percentage );

Image_Data_gray_80_2 $\{$ i i $\}=I_{-} n$;

Image_Data_gray_Reshape_80_2 $\{\mathrm{i} i\}=$ double (reshape (

Image_Data_gray_80_2 2 i i $\}, 86 * 83,1))$;

$\%$ With $85 \%$ Disturbance

I_n $=$ Image_Data_gray $\{$ i i $\}$;

$\%$ Now the program is repeated for $85 \%$ noise.

Noise_Percentage $=85$;

I_n=Image_Noisy (I_n, Noise_Percentage );

Image_Data_gray_85\{ i i $\}=I_{-} n$;

Image_Data_gray_Reshape_85\{ i i $\}=$ double(reshape ( 
Image_Data_gray_ $85\{$ i i $\}, 86 * 83,1))$;

$\%$ With 85_2 Disturbance

$\mathrm{I}_{-} \mathrm{n}=$ Image_Data_gray $\{\mathrm{i} i\}_{\text {; }}$

Noise_Percentage $=85 ;$

I_n=Image_Noisy ( I_n, Noise_Percentage ) ;

Image_Data_gray_85_2 $\{$ i i $\}=I_{-} n$;

Image_Data_gray_Reshape_85_2 $\{\mathrm{i} i\}=$ double (reshape (

Image_Data_gray_85_2\{ i i $\}, 86 * 83,1))$;

$\%$ With 90\% Disturbance

I_n $=$ Image_Data_gray $\{$ i i $\}$;

$\% \%$ Now the program is repeated for $90 \%$ noise.

Noise_Percentage $=90 ;$

I_n=Image_Noisy ( I_n, Noise_Percentage ) ;

Image_Data_gray_90\{i $\}=I_{-} n$;

Image_Data_gray_Reshape_90 $\{\mathrm{i} i\}=$ double $($ reshape (

Image_Data_gray_90 $\{$ i i $\}, 86 * 83,1))$;

$\%$ With $90 \_2$ Disturbance

I_n $=$ Image_Data_gray $\{\mathrm{i} i\}$;

Noise_Percentage $=90 ;$

I_n=Image_Noisy (I_n, Noise_Percentage );

Image_Data_gray_90_2 $\{$ i i $\}=I_{-} n$;

Image_Data_gray_Reshape_90_2 $\{\mathrm{i} i\}=$ double (reshape (

Image_Data_gray_90_2\{ i i $\}, 86 * 83,1))$;

$\%$ With 95\% Disturbance

$I_{-} n=$ Image_Data_gray $\{$ i $i\} ;$ 
258 259

$\% \%$ Now the program is repeated for $95 \%$ noise.

Noise_Percentage $=95 ;$

I_n=Image_Noisy ( I_n, Noise_Percentage ) ;

Image_Data_gray_95 $\{\mathrm{ii}\}=\mathrm{I}_{-} \mathrm{n}$;

Image_Data_gray_Reshape_95 $\{$ i i $\}=$ double (reshape (

Image_Data_gray_95\{ i i $\}, 86 * 83,1))$;

$\%$ With 95\%_2 Disturbance

I_n $=$ Image_Data_gray $\{$ i i $\}$;

Noise_Percentage $=95 ;$

I_n=Image_Noisy ( I_n, Noise_Percentage ) ;

Image_Data_gray_95_2 $\{\mathrm{i}$ i $\}=\mathrm{I}_{-} \mathrm{n}$;

Image_Data_gray_Reshape_95_2 $\{$ i i $\}=$ double (reshape (

Image_Data_gray_95_2\{ i i $\}, 86 * 83,1))$;

$\%$ It is worth noting the number of the sign is given by 8

bits at the output of the network. Now ewe need to prepare the desired output which is equal to the

$\%$ original picture for all the noisy pictures which have been generated above.

Counter_Bin $=\operatorname{dec} 2$ bin $($ ii, 8$)$;

Out_Mat $=[$ bin2dec $($ Counter_Bin $(1))$

bin2dec (Counter_Bin (2))

bin2dec (Counter_Bin (3))

bin 2 dec (Counter_Bin (4))

bin2dec (Counter_Bin (5)) 
272

$$
\begin{aligned}
& \text { bin2dec(Counter_Bin }(6)) \\
& \text { bin2dec(Counter_Bin }(7)) \\
& \text { bin2dec(Counter_Bin (8)) ] ; }
\end{aligned}
$$

$\%$ And this is to structure the input data which consists of one original picture in the first vector and the noisy pictures following /

$$
\begin{aligned}
& \operatorname{Input}(:, \mathrm{j} j: \mathrm{j} j+38)=[\text { Image_Data_gray_Reshape }\{\mathrm{i} i\} \\
& \text { Image_Data_gray_Reshape_5 }\{\text { i i }\} \\
& \text { Image_Data_gray_Reshape_5_2 } 2 \text { i i }\} \\
& \text { Image_Data_gray_Reshape_10 } 10 \text { i i }\} \\
& \text { Image_Data_gray_Reshape_10_2 }\{\text { i i }\} \\
& \text { Image_Data_gray_Reshape_ } 15\{\text { i i }\} \\
& \text { Image_Data_gray_Reshape_15_2\{ i i }\} \\
& \text { Image_Data_gray_Reshape_20\{ i i }\} \\
& \text { Image_Data_gray_Reshape_20_2\{ i i }\} \ldots \\
& \text { Image_Data_gray_Reshape_25\{ i i }\} \\
& \text { Image_Data_gray_Reshape_25_2\{ i i }\} \\
& \text { Image_Data_gray_Reshape_30 }\{\mathrm{i} \text { i }\} \\
& \text { Image_Data_gray_Reshape_30_2 } 2 \text { i i }\} \\
& \text { Image_Data_gray_Reshape_35\{ i i }\} \\
& \text { Image_Data_gray_Reshape_35_2\{ i i }\} \\
& \text { Image_Data_gray_Reshape_40\{ i i }\} \\
& \text { Image_Data_gray_Reshape_40_2 } 2 \mathrm{i} \text { i }\}
\end{aligned}
$$


Image_Data_gray_Reshape_45\{ i i $\} \ldots$

Image_Data_gray_Reshape_45_2\{ i i $\}$

Image_Data_gray_Reshape_50\{ i i $\}$

Image_Data_gray_Reshape_50_2\{ i i $\}$

Image_Data_gray_Reshape_55\{ i i $\}$

Image_Data_gray_Reshape_5 $522\{\mathrm{i}$ i $\}$

Image_Data_gray_Reshape_60\{ i i $\}$

Image_Data_gray_Reshape_60_2\{ i i $\}$

Image_Data_gray_Reshape_65\{ i i $\}$

Image_Data_gray_Reshape_65_2\{ i i $\}$. . Image_Data_gray_Reshape_70 i i $\}$

Image_Data_gray_Reshape_70_2 2 i i $\}$

Image_Data_gray_Reshape_75\{ i i $\}$

Image_Data_gray_Reshape_ $75 \_2\{\mathrm{i}$ i $\}$

Image_Data_gray_Reshape_80\{ i i $\}$

Image_Data_gray_Reshape_80_2\{ i i $\}$

Image_Data_gray_Reshape_85\{ i i $\}$

Image_Data_gray_Reshape_85_2\{ i i $\}$

Image_Data_gray_Reshape_90 $\{$ i i $\}$

Image_Data_gray_Reshape_90_2\{ i i $\}$

Image_Data_gray_Reshape_95\{ i i $\}$

Image_Data_gray_Reshape_95_2\{ i i $\}$; 
our pictures, so we continue for the next sign from the column 39 by using this command.

280

281

282

283

284 end

${ }_{285} \%$ It is worth noting moreover to the 58 original signs, we need one more sign to show it when the network can not match the correct picture. At this stage ii=58 and 286 $\%$ then the 59 th picture would be the wrong sign which has been added in our bank of pictures.

$287 \quad \mathrm{i}$ i $=\mathrm{i} \mathrm{i}+1$;

288 Image_Data $\{$ i i $\}=\operatorname{imread}\left(\left[\right.\right.$ ' Guide_Pictures $\backslash P_{-}$',, $\operatorname{num} 2 \operatorname{str}($ ii $)$, ' . jpg'] ) ;

289 I_n $=$ rgb2gray (Image_Data $\{$ i i $\})$;

290 Image_Data_gray $\{$ i i $\}=I_{-} n$;

291 end 


\section{Noisy Image}

$1 \%$ In this program we use a function to make the original pictures noisy!

2

3 function Noisy_Output_Picture=Image_Noisy (Input_Picture , Noise_Percentage) \% The program receives inputs and make them noisy and send them out.

${ }_{4} \%$ In the following lines we check if the givennumbers are not correct, (negative value or something more than 100\%) we use the boundary conditions.

5 if Noise_Percentage $<=0^{2}$

$6 \quad$ Noise_Percentage $=1$;

7 elseif Noise_Percentage $>100$

$8 \quad$ Noise_Percentage $=100$

9 end

10

$11 \%$ In the following lines we prevent any possibility of repeated random numbers to make sure that our data set dose not include repeated numbers.

12 ss $=$ RandStream ('mt19937ar', 'Seed ',0);

13 Rand_Home $=$ randperm $(\mathrm{ss}, 86 * 83$, floor $(($ Noise_Percentage $/ 100)$ $* 86 * 83)) ; \%$ Here when we want $50 \%$ noise to be included it means that $0.5 * 86 * 83$ pixelex must be noisy .

14

$15 \%$ In the following lines we indicate the position of the 
pixel which needs to be noisy.

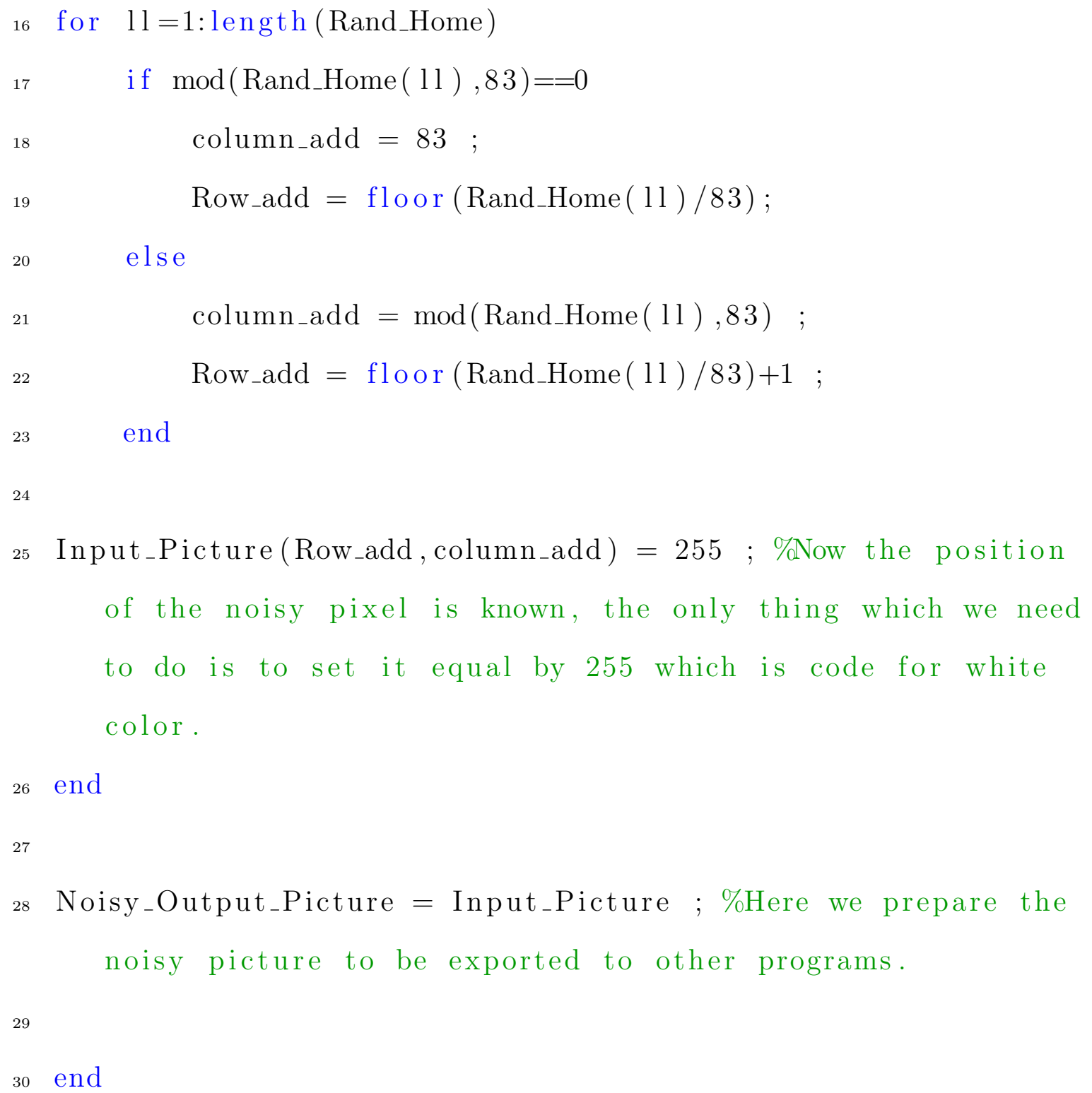




\section{References}

[1] K. Biswas and V. Muthukkumarasamy, "Securing smart cities using blockchain technology," in Proc. IEEE 18th International Conference on High Performance Computing and Communications, pp. 1392-1393, 2016.

[2] S. Ibba, A. Pinna, M. Seu, and F. E. Pani, "Citysense: Blockchain-oriented smart cities," in Proc. the XP2017 Scientific Workshops, pp. 12:1-12:5, 2017.

[3] website: http://nsercdiva.com/index.php.

[4] S. Rowan, M. Clear, M. Gerla, M. Huggard, and C. M. Goldrick, "Securing vehicle to vehicle communications using blockchain through visible light and acoustic side-channels," CoRR, vol. abs/1704.02553, 2017.

[5] A. Dorri, S. S. Kanhere, R. Jurdak, and P. Gauravaram, "Blockchain for iot security and privacy: The case study of a smart home," in Proc. 2017 IEEE International Conference on Pervasive Computing and Communications Workshops (PerCom Workshops), pp. 618-623, March 2017.

[6] C. Felipe, A. Boukerche, L. Villas, A. Viana, and A. Loureiro, "Data communication in vanets: A survey, challenges and applications," Ad Hoc Networks, 03 2014. 
[7] Y. Roper, M. Rowland, Z. Kachick, W. McGIll, V. Nanayakkara, D. Young, and R. Whale, "Implications of traffic sign recognition (tsr) systems for road operators," 2018.

[8] website: www.ictc-ctic.ca/autonomous vehicles and the future of work in Canada.

[9] P. Alessandra, N. Scarpato, and M. Brilli, "Industry 4.0 revolution in autonomous and connected vehicle a non-conventional approach to manage big data," Journal of Theoretical and Applied Information Technology, vol. Vol 96, 012018.

[10] D. F. Specht, "A general regression neural network," IEEE Transactions on Neural Networks, vol. 2, pp. 568-576, Nov 1991.

[11] N. Aljeri and A. Boukerche, "An efficient handover trigger scheme for vehicular networks using recurrent neural networks," pp. 85-91, 112019.

[12] N. Lu, N. Cheng, N. Zhang, X. Shen, and J. W. Mark, "Connected vehicles: Solutions and challenges," IEEE Internet of Things Journal, vol. 1, pp. 289-299, Aug 2014.

[13] P. K. Agarwal, M. A. Aiello, M. A. Aiello, S. H. Hilfinger, D. W. Kantaros, and E. T. R. Jr, "2017 connected cars autonomous vehicles survey," 2017.

[14] K. Shafiee, A. Attar, and V. C. M. Leung, "Optimal distributed vertical handoff strategies in vehicular heterogeneous networks," IEEE Journal on Selected Areas in Communications, vol. 29, pp. 534-544, March 2011. 
[15] S. E. Shladover, "Connected and automated vehicle systems: Introduction and overview," Journal of Intelligent Transportation Systems, vol. 22, no. 3, pp. 190$200,2018$.

[16] R. W. L. Coutinho and A. Boukerche, "Modeling and analysis of a shared edge caching system for connected cars and industrial iot-based applications," IEEE Transactions on Industrial Informatics, pp. 1-1, 2019.

[17] A. Petrovskaya and S. Thrun, "Model based vehicle detection and tracking for autonomous urban driving," Autonomous Robots, vol. 26, pp. 123-139, Apr 2009.

[18] A. S. Huang, Lane Estimation for Autonomous Vehicles using Vision and LIDAR. PhD thesis, MASSACHUSETTS INSTITUTE OF TECHNOLOGY, 2010.

[19] Z. Luo, LiDAR Based Perception System: Pioneer Technology for Safety Driving. PhD thesis, McMaster University, 2017.

[20] "Automative sensors and electronics expo 2017." http://www.automotivesensors2017.com.

[21] T. L. Willke, P. Tientrakool, and N. F. Maxemchuk, "A survey of inter-vehicle communication protocols and their applications," IEEE Communications Surveys Tutorials, vol. 11, pp. 3-20, Second 2009.

[22] M. Francisconi, "An explorative study on blockchain technology in application to port logistics," Master's thesis, Delft University of Technology, Netherlands, 82017.

[23] L. Xu, L. Y. Wang, G. Yin, and H. Zhang, "Communication information structures and contents for enhanced safety of highway vehicle platoons," IEEE Trans. Veh. Tech., vol. 63, pp. 4206-4220, Nov. 2014. 
[24] C. F. H. K. Bauche-Dit Morency Patrick, Caminiti Domenic, "Cybersecurity of connected and autonomous vehicles," tech. rep., Dept. of Information Technology, University of Carleton, 2018. Project Final Report.

[25] "Automated vehicles for safety." https://www.nhtsa.gov/technologyinnovation/automated-vehicles-safety.

[26] A. Mammeri, Y. Zhao, A. Boukerche, A. J. Siddiqui, and B. Pekilis, "Design of a semi-supervised learning strategy based on convolutional neural network for vehicle maneuver classification," in 2019 IEEE International Conference on Wireless for Space and Extreme Environments (WiSEE), pp. 65-70, Oct 2019.

[27] "Future of driving." https://www.tesla.com/en ${ }_{C} A /$ autopilot.

[28] "Audi a8 ai traffic jam pilot.." https://www.audi-technology-portal.de/en/electricselectronics/driver-assistant-systems/audi-a8-audi-ai-traffic-jam-pilot.

[29] A. J. Hawkins, "Waymo is first to put fully self-driving cars on us roads without a safety driver." https://www.theverge.com/2017/11/7/16615290/waymo-self-drivingsafety-driver-chandler-autonomous.

[30] website: https://en.wikipedia.org/wiki/Openpilot.

[31] C. Ai, "How does openpilot work?." https://medium.com/@comma $i / h o w-d o e s-$ openpilot - work - c7076d4407b3.

[32] website: https://github.com/commaai.

[33] M. Rouse, "What is dedicated short-range communication (dsrc)? - definition from whatis.com." https://whatis.techtarget.com/definition/dedicated-short-rangecommunication-DSRC. 
[34] Y. Song, R. Yu, Y. Fu, L. Zhou, and A. Boukerche, "Multi-vehicle cooperative positioning correction framework based on vehicular blockchain," DIVANet '19, (New York, NY, USA), p. 23-29, Association for Computing Machinery, 2019.

[35] A. Holzer, S. Maaroufi, and S. Pierre, "Dymes: A dynamic messaging service for vanets," in 2010 IEEE 6th International Conference on Wireless and Mobile Computing, Networking and Communications, pp. 513-520, Oct 2010.

[36] website: https://cohdawireless.com/solutions/hardware/mk5-obu/.

[37] website: https://app.box.com/file/2925813611161.

[38] website: https://github.com/commaai/openpilotl.

[39] Y. Kim and B. Toomajian, "Hand gesture recognition using micro-doppler signatures with convolutional neural network," IEEE Access, vol. 4, pp. 7125-7130, 2016.

[40] Y. Sun, D. Liang, X. Wang, and X. Tang, "Deepid3: Face recognition with very deep neural networks," CoRR, vol. abs/1502.00873, 2015.

[41] S. Kouamo and C. Tangha, "Fingerprint recognition with artificial neural networks: Application to e-learning," Intelligent Learning Systems and Applications, 2016.

[42] G. C. Goodwin and K. S. Sin, Adaptive filtering prediction and control. Courier Corporation, 2014.

[43] K. S. Narendra and K. Parthasarathy, "Identification and control of dynamical systems using neural networks," IEEE Transactions on neural networks, vol. 1, no. 1, pp. $4-27,1990$.

[44] J. M. Zurada, Introduction to artificial neural systems, vol. 8. West publishing company St. Paul, 1992. 
[45] J. A. Freeman and D. M. Skapura, Neural Networks: Algorithms, Applications, and Programming Techniques. Redwood City, CA, USA: Addison Wesley Longman Publishing Co., Inc., 1991.

[46] J. Yang, L. Wang, Y. Wang, and T. Guo, "A novel memristive hopfield neural network with application in associative memory," Neurocomputing, vol. 227, pp. 142-148, 2017.

[47] website: https://datascience.stackexchange.com/questions/6675/identifying-inputsequence-using-neural-network.

[48] "Character recognition using hopfield networks." website: https://pmatigakis.wordpress.com/2014/01/18/character-recognition-using-hopfieldnetworks/.

[49] website: https://en.wikipedia.org/wiki/Radial ${ }_{b}$ asis $_{f} u n c t i o n$.

[50] F. Schwenker, H. A. Kestler, and G. Palm, "Three learning phases for radial-basisfunction networks," Neural Netw., vol. 14, pp. 439-458, May 2001.

[51] D. F. Specht, "A general regression neural network," IEEE Transactions on Neural Networks, vol. 2, pp. 568-576, Nov 1991.

[52] H.-Z. Li, S. Guo, C.-J. Li, and J.-Q. Sun, "A hybrid annual power load forecasting model based on generalized regression neural network with fruit fly optimization algorithm," Knowledge-Based Systems, vol. 37, pp. 378-387, 2013.

[53] H. K. Cigizoglu, "Generalized regression neural network in monthly flow forecasting," Civil Engineering and Environmental Systems, vol. 22, no. 2, pp. 71-81, 2005.

[54] website: https://en.wikipedia.org/wiki/General_regression_neural_network. 
[55] website: https://www.mathworks.com/help/deeplearning/ug/generalized-regressionneural-networks.html.

[56] S. B. Wali, M. A. Abdullah, M. A. Hannan, A. Hussain, S. A. Samad, P. J. Ker, and M. B. Mansor, "Vision-based traffic sign detection and recognition systems: current trends and challenges," Sensors, vol. 19, no. 9, p. 2093, 2019.

[57] M. Miah and M. Bhashani, "A real time road sign recognition using neural network," International Journal of Computer Applications, vol. 114, no. 13, 2015.

[58] S. Hamdi, H. Faiedh, C. Souani, and K. Besbes, "Road signs classification by ann for real-time implementation," in 2017 International Conference on Control, Automation and Diagnosis (ICCAD), pp. 328-332, Jan 2017.

[59] K. Tohidul Islam and R. Gopal Raj, "Real-time (vision-based) road sign recognition using an artificial neural network," Sensors, vol. 17, 2017.

[60] F. Shao, X. Wang, F. Meng, T. Rui, D. Wang, and J. Tang, "Real-time traffic sign detection and recognition method based on simplified gabor wavelets and cnns," Sensors, vol. 18, p. 3192, 2018.

[61] J. Li and Z. Wang, "Real-time traffic sign recognition based on efficient cnns in the wild," IEEE Transactions on Intelligent Transportation Systems, vol. 20, pp. 975-984, March 2019 .

[62] X. Du, M. H. Ang, and D. Rus, "Car detection for autonomous vehicle: Lidar and vision fusion approach through deep learning framework," in 2017 IEEE/RSJ International Conference on Intelligent Robots and Systems (IROS), pp. 749-754, Sep. 2017. 
[63] S. Zhang, C. Wang, Z. Yang, C. Wen, J. Li, and C. Yang, "Traffic sign timely visual recognizability evaluation based on $3 \mathrm{~d}$ measurable point clouds," CoRR, vol. abs/1710.03553, 2017.

[64] website: https://www.blickfeld.com/what-is-lidar/.

[65] website: [https://en.wikipedia.org/wiki/Lidar.

[66] J. Landa and D. Prochazka, "Automatic road inventory using lidar," 2014.

[67] F. Ghallabi, G. El-Haj-Shhade, M. Mittet, and F. Nashashibi, "Lidar-based road signs detection for vehicle localization in an hd map," in 2019 IEEE Intelligent Vehicles Symposium (IV), pp. 1484-1490, June 2019.

[68] website: https://www.engadget.com/2017/11/29/velodyne-lidar-helps-self-drivingcars-survive-the-highway/.

[69] C. Ai and Y. J. Tsai, "Critical assessment of an enhanced traffic sign detection method using mobile lidar and ins technologies," 2015.

[70] Y. Li, J. Fan, Y. Huang, and Z. Chen, "Lidar-incorporated traffic sign detection from video log images of mobile mapping system," 2016.

[71] website: https://www.engadget.com/2017/11/29/velodyne-lidar-helps-self-drivingcars-survive-the-highway/.

[72] website: https://en.m.wikipedia.org/wiki/Velodyne ${ }_{L} i D A R$ cite $_{n}$ ote $-U S A_{T}$ oday -5$]$.

[73] S. Wu, C. Wen, H. Luo, Y. Chen, C. Wang, and J. Z. Li, "Using mobile lidar point clouds for traffic sign detection and sign visibility estimation," 2015 IEEE International Geoscience and Remote Sensing Symposium (IGARSS), pp. 565-568, 2015. 
[74] G. Neel, A. Darwesh, Q. Le, and S. Saripalli, "Autonomous shuttles for last-mile connectivity," 2019.

[75] website: https://github.com/nutonomy/second.pytorch. 